\title{
Quantum Link Homology via Trace Functor I
}

\author{
Anna Beliakova Krzysztof K. Putyra Stephan M. Wehrli
}

\begin{abstract}
Motivated by topology, we develop a general theory of traces and shadows for an endobicategory, which is a pair: bicategory $\mathbf{C}$ and endobifunctor $\Sigma: \mathbf{C} \longrightarrow \mathbf{C}$. For a graded linear bicategory and a fixed invertible parameter $q$, we quantize this theory by using the endofunctor $\Sigma_{q}$ such that $\Sigma_{q} \alpha:=q^{-\operatorname{deg} \alpha} \Sigma \alpha$ for any 2-morphism $\alpha$ and coincides with $\Sigma$ otherwise.

Applying the quantized trace to the bicategory of Chen-Khovanov bimodules we get a new triply graded link homology theory called quantum annular link homology. If $q=1$ we reproduce Asaeda-Przytycki-Sikora (APS) homology for links in a thickened annulus. We prove that our homology carries an action of $\boldsymbol{U}_{q}\left(\mathfrak{s t}_{2}\right)$, which intertwines the action of cobordisms. In particular, the quantum annular homology of an $n$-cable admits an action of the braid group, which commutes with the quantum group action and factors through the Jones skein relation. This produces a nontrivial invariant for surfaces knotted in four dimensions. Moreover, a direct computation for torus links shows that the rank of quantum annular homology groups does depend on the quantum parameter $q$.
\end{abstract}

\section{Contents}

1 Introduction 2

2 Generalized traces $\quad 13$

2.1 Twisted traces . . . . . . . . . . . . . . . . . . . 13

2.2 Triangulated traces . . . . . . . . . . . . . . . . . . 16

2.3 Deformation of twisted traces . . . . . . . . . . . . . . 17

2.4 Examples . . . . . . . . . . . . . . . . . . . . . . . . . . 19

3 Traces of bicategories $\quad 25$

3.1 Categorified traces . . . . . . . . . . . . . . . . . 25

3.2 The universal property of the horizontal trace . . . . . . . . . . . 27

3.3 Functoriality . . . . . . . . . . . . . . . . . . . 28

3.4 A connection with the vertical trace . . . . . . . . . . . . 30

3.5 Decategorification . . . . . . . . . . . . . . . . 31

3.6 Categorified Lefschetz traces . . . . . . . . . . . . . . . . . . . . . . . . . . . . . . . . . . .

3.7 Deformation of categorified traces . . . . . . . . . . . . . 34

3.8 Examples . . . . . . . . . . . . . . . . . . . . 35 
4.1 Twisted Hochschild-Mitchell homology . . . . . . . . . . . . . . . . . . . 41

4.2 Twisted Hochschild-Mitchell complex is a preshadow . . . . . . . . . . . 43

$4.3 \quad K$-theoretic invariance . . . . . . . . . . . . . . . . 45

4.4 An application to Hochschild homology of algebras . . . . . . . . . . 46

5 Khovanov Homology 48

5.1 The formal bracket . . . . . . . . . . . . . . . . . . . . 48

5.2 Khovanov homology for links in $\mathbb{R}^{3} \ldots \ldots \ldots \ldots 1$

5.3 Annular link homology . . . . . . . . . . . . . . . . . . . . . . 52

5.4 Homology for links in a thickened Möbius band . . . . . . . . . . 54

5.5 Chen-Khovanov homology for tangles . . . . . . . . . . . . . 55

6 Quantization of the annular link homology 61

6.1 The action of $\mathfrak{s l}_{2}$ revisited . . . . . . . . . . . . . . . 61

6.2 Deformation of the annular skein category . . . . . . . . . . . . 61

6.3 Quantum annular homology . . . . . . . . . . . . . . 65

6.4 Homology for $(2, n)$ torus links . . . . . . . . . . . 67

7 Applications and generalizations $\quad 69$

7.1 Invariants for annular link cobordisms . . . . . . . . . . . . . . . 69

7.2 An action of tangles on cablings . . . . . . . . . . . . . 71

7.3 Quantum homology of links in a thickened Möbius band . . . . . . . . 73

7.4 Annular twistors . . . . . . . . . . . . . . . . . . 75

7.5 Generalized annular homology . . . . . . . . . . . . . 75

$\begin{array}{ll}\text { A Background survey } & 76\end{array}$

A.1 Representations of $\boldsymbol{U}_{q}\left(\mathfrak{s l}_{2}\right) \ldots \ldots \ldots \ldots$. . . . . . . . . . . . . . . . . . . . . . 76

A.2 Knots and tangles . . . . . . . . . . . . . . . . . 78

A.3 Constructions on categories . . . . . . . . . . . . . . 78

A.4 Bicategories . . . . . . . . . . . . . . . . . 80

\section{Introduction}

\section{Background and Overview}

Trace are an important source of topological invariants. Given a category $\mathscr{C}$, a trace is a collection of functions $t_{x}$ defined on the endomorphism spaces $\mathscr{C}(x, x)$, indexed by objects $x \in \mathscr{C}$, such that for any pair of composable morphisms $x \stackrel{g}{\longleftarrow} y \stackrel{f}{\longleftarrow} x$ in $\mathscr{C}$ the relation

$$
t_{x}(g \circ f)=t_{y}(f \circ g)
$$

holds. When $\mathscr{C}$ is the category of vector spaces, then the above relation determines the trace uniquely (up to a scalar) as the sum of the diagonal entries of a matrix representing the endomorphism. The well-known Reshetikhin-Turaev package of link invariants is obtained by applying traces to linear endomorphisms associated with tangles. In this context, the cyclicity relation is interpreted topologically as passing to the annular closure of a tangle. 
Every category admits the universal trace, defined as

$$
\operatorname{Tr}(\mathscr{C}):=\coprod_{x \in \operatorname{Ob}(\mathscr{C})} \mathscr{C}(x, x) / g \circ f \sim f \circ g
$$

where $f$ and $g$ run through all pairs of composable morphisms. Any trace on $\mathscr{C}$ factorizes through $\operatorname{Tr}(\mathscr{C})$. If $\mathscr{C}$ is an additive category, then it receives the Chern character map from the Grothendieck group $K_{0}(\mathscr{C})$, and hence can be considered as an alternative decategorification functor [BGHL14, BHLZ14]. Another important feature of the trace is its functoriality.

Let us consider the category $\mathscr{T}$ an of tangles, objects of which are points on the $x$-axis in $\mathbb{R}^{2}$ and morphisms are tangles in $\mathbb{R}^{2} \times I$. Any functor $F: \mathscr{T}$ an $\longrightarrow \mathscr{A}$ induces a map

$$
\operatorname{Tr}(F): \operatorname{Tr}(\mathscr{T} a n) \longrightarrow \operatorname{Tr}(\mathscr{A}),
$$

which is a universal annular link invariant associated with $\mathscr{A}$. In the Reshetikhin-Turaev case, $\mathscr{A}$ is a category of representations of a quantum group.

For quantum $\mathfrak{s l}_{2}$ the Reshetikhin-Turaev construction was categorified by Chen and Khovanov in [CK14]. For this purpose Tan was extended to a bicategory Tan with $2-$ morphisms given by tangle cobordisms. Chen and Khovanov defined a projective bifunctor

$$
\widetilde{\mathbf{F}}_{C K}: \operatorname{Tan} \longrightarrow \operatorname{Com}_{/ h}^{b}(\text { gBirep})
$$

valued in the homotopy category of graded sweet bimodules ${ }^{1}$ Birep. Here, 'projective' means that the bifunctor is defined on 2-morphisms only up to a sign.

In this paper, we develop a theory of traces in endobicategories and apply it to the Chen-Khovanov construction to obtain a new triply graded quantum annular link homology theory. The sign issue will be resolved in a second paper [BHPW18] by redefining our and Chen-Khovanov's constructions using $\mathfrak{g l}_{2}-$ foams. We will use it in [BHPW] to show that all known definitions of the colored Khovanov homology coincide in the quantum annular setting when the quantization parameter is not a root of unity.

\section{Traces in Endobicategories}

Let us first present our categorical results. There are two ways to define traces on a bicategory: applying (1.1) to morphism categories results in the vertical trace, and a more general horizontal trace was defined in [BHLZ14]. Let us visualize this construction for the bicategory $\mathbf{C o b}$, the objects of which are points on a line, 1-morphisms are flat tangles in $\mathbb{R} \times I$, and 2-morphisms are cobordisms in $(\mathbb{R} \times I) \times I$. The horizontal trace of a cobordism between two different $(2,2)$-tangles is shown in Figure 1, where 1-morphisms are depicted horizontally. Roughly speaking, the horizontal trace is obtained by imposing the cyclicity relation on the horizontal composition of 1 -morphisms in a way compatible with 2-morphisms (see Section 3 for more details).

An analogue of a trace function for a bicategory is called shadow, and was defined by Ponto and Shulman [PS13]. In this paper, inspired by topology, we further generalize the notions of the universal trace of a category, as well as vertical and horizontal traces of a bicategory. Recall that a surface bundle $M$ over a circle with monodromy

\footnotetext{
${ }^{1}$ Following [Kh02] we say that a bimodule is sweet if it is finitely generated and projective when considered as a left and as a right module separately.
} 

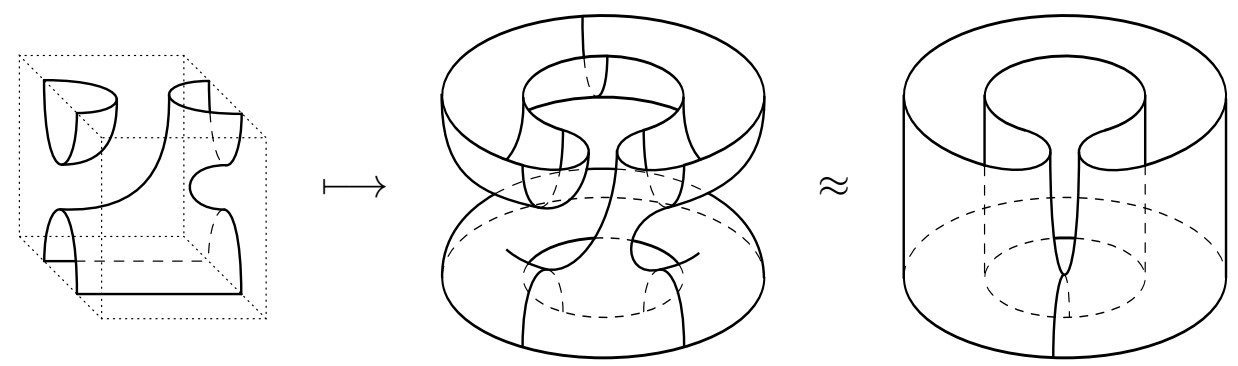

Figure 1: A horizontal trace of a cobordism with corners.

$\phi$ is constructed from a thickened surface $F \times I$ by gluing $F \times\{0\}$ to $F \times\{1\}$ along the diffeomorphism $\phi$ :

$$
M:=F \times I /(p, 1) \sim(\phi(p), 0) .
$$

To mimic this construction on a more abstract level, we consider a pair: a category $\mathscr{C}$ and an endofunctor $\Sigma: \mathscr{C} \longrightarrow \mathscr{C}$. Then a $\Sigma$-twisted trace of $\mathscr{C}$ is defined as

$$
\operatorname{Tr}(\mathscr{C}, \Sigma):=\coprod_{x \in \operatorname{Ob}(\mathscr{C})} \mathscr{C}(x, \Sigma x) / \Sigma f \circ g \sim g \circ f
$$

where $f$ and $g$ run through all pairs of composable morphisms $\Sigma x \stackrel{g}{\longleftarrow} y \stackrel{f}{\longleftarrow} x$ in $\mathscr{C}$. This construction is functorial with respect to $(\mathscr{C}, \Sigma)$ and universal. We recover the usual trace of $\mathscr{C}$ when $\Sigma$ is the identity functor.

Let Tan be the category of isotopy classes of tangles in $\mathbb{R}^{2} \times I$. The rotation of the plane induces an endofunctor $\Sigma$ on $\mathscr{T} a n$, for which $\operatorname{Tr}(\mathscr{T} a n, \Sigma)$ recovers links in the thickened Möbius band.

We observe that any natural transformation of the endofunctor on an endocategory induces a new trace function. For example, fixing a framed $(1,1)$-tangle $\tau$ we can define a natural transformation of the identity functor of $\mathcal{T}$ an by sending a collection of $n$ points to the $n$-cabling $\tau^{n}$, viewed as a morphism in $\mathscr{T} a n$. It is a natural transformation because of the equality $\tau^{m} \circ T=T \circ \tau^{n}$, which holds for any $(m, n)$-tangle $T$. The same euqality also implies that the map sending an $(n, n)$-tangle $T$ to the annular closure of the composition $T \circ \tau^{n}$ is well-defined trace function on $\mathscr{T}$ an. In particular, one can compose any $(n, n)$-tangle with a full twist before closing. Hence, traces on endocategories of tangles encode information not only about a simple annular closure $\widehat{T}$ of any $(n, n)$-tangle $T$, but also on all satellites of $\widehat{T}$.

Triangulated categories are treated separately. They admit triangulated traces, which are additive with respect to homomorphisms of distinguished triangles, see (2.14). We show that twisted triangulated traces on the homotopy endocategory of bounded complexes $C_{o m} / h(\mathscr{C})$ are Lefschetz traces, which are unique extensions of twisted traces on $\mathscr{C}$. In particular, there is an isomorphism between the universal triangulated trace $\operatorname{Tr}^{\triangle}\left(\operatorname{Com}_{/ h}^{b}(\mathscr{C}), \Sigma\right)$ and $\operatorname{Tr}(\mathscr{C}, \Sigma)$.

Twisted traces admit a quantization when $\mathscr{C}$ is pregraded. ${ }^{2}$ For that fix an invertible parameter $q$ and consider the endofunctor $\Sigma_{q}$ that coincides with $\Sigma$ on objects, but $\Sigma_{q} f:=q^{-|f|} \Sigma f$ for a homogeneous morphism $f$ of degree $|f|$. This results in the quantum universal trace

$$
\operatorname{Tr}_{q}(\mathscr{C}, \Sigma):=\operatorname{Tr}\left(\mathscr{C}, \Sigma_{q}\right)=\coprod_{x \in \operatorname{Ob}(\mathscr{C})} \mathscr{C}(x, \Sigma x) / \Sigma f \circ g-q^{|f|} g \circ f \cdot
$$

\footnotetext{
${ }^{2}$ A category is pregraded if its sets of morphisms are graded modules.
} 
Further, we recall and generalize of quantize traces that appear commonly in algebraic and topological contexts, including the Hattori-Stallings trace, the Lefschetz number, traces in pivotal categories, and various closures of tangles.

We also investigate the quantum analogue of the Hochschild-Mitchell homology for endocategories and prove the quantum version of the $K$-theoretic equivalence, generalizing that of Keller [Ke98]. Roughly speaking, it states that the homotopy class of the chain map (between quantum Hochschild-Mitchell chain complexes) induced by a bimodule $M$ depends only on the image of $M$ in the Grothendieck group, see Proposition 4.9. This very powerful tool is used later to find the quantum Hochschild homology of Chen-Khovanov algebras.

To categorify the constructions described above, we define the notions of a $\Sigma$-twisted preshadow and a $\Sigma$-twisted horizontal trace for a bicategory $\mathbf{C}$ and its endobifunctor $\Sigma$, generalizing those introduced in [PS13] and [BHLZ14]. The pair $(\mathbf{C}, \Sigma)$ is called an endobicategory. We prove that any twisted preshadow factors through the twisted horizontal trace under the mild assumption that $\mathbf{C}$ has left duals. Hence, for bicategories with duals the horizontal trace $h \operatorname{Tr}(\mathbf{C}, \Sigma)$ is a universal preshadow.

When $\mathbf{C}$ is pregraded, a deformation of $\Sigma$, defined by setting $\Sigma_{q} f:=q^{-|f|} \Sigma f$ for any homogeneous 2-morphism $f$ of degree $|f|$, leads to a theory of quantum preshadows and a quantum horizontal trace.

The horizontal trace can be viewed as a categorification of the trace of an endocategory in the following sense. Let $\Pi: n$ Cat $\longrightarrow(n-1)$ Cat be the decategorification functor, which forgets the $n$-morphisms and identifies isomorphic $(n-1)$-morphisms. Then there is a natural bijection of sets $\Pi(\mathrm{h} \operatorname{Tr}(\mathbf{C}, \Sigma)) \approx \operatorname{Tr}(\Pi \mathbf{C}, \Pi \Sigma)$ for any small endobicategory $(\mathbf{C}, \Sigma)$ with both left and right duals (see Section 3.5).

Applying the construction (1.3) to morphism categories in $\mathbf{C}$ results in the vertical trace $v \operatorname{Tr}(\mathbf{C}, \Sigma)$. There is a functor from the vertical to the horizontal trace of an endobicategory, which is full and faithful, but not necessarily surjective on objects. Quite often a preshadow can be restricted to a collection of traces on morphisms sets of the vertical trace, for instance the shadow computing coinvariants in bimodules restricts to the Hattori-Stallings trace (see Section 3.8.2).

Of particular interest to us are preshadows on bicategories of complexes. We extend every preshadow on $(\mathbf{C}, \Sigma)$ to a preshadow on $\operatorname{Com}_{/ h}^{b}(\mathbf{C}, \Sigma)$, which - by the analogy to traces - we call the Lefschetz preshadow. These satisfy a higher analogue of additivity with respect to distringuished triangles (see Proposition 3.17 for a precise statement).

Again we provide many examples of preshadows on endobicategories. Among them are twisted spaces of coinvariants, quantum Hochschild homology, and component-wise Hochschild homology of a complex of bimodules (which is an example of a Lefschetz preshadow mentioned above). Furthermore, we compute explicitly twisted horizontal traces of $\operatorname{Tan}(F)$, the bicategory of oriented tangles in a thickened surface $F$ and oriented tangle cobordisms (see Appendix A.2 for a precise definition).

Theorem A. Let $M$ be a surface bundle with fiber $F$ and monodromy $\phi \in \operatorname{Diff}(F)$. There is an equivalence of categories

$$
\mathrm{h} \operatorname{Tr}\left(\operatorname{Tan}(F), \phi_{*}\right) \simeq \mathscr{L i n k s}(M)
$$

where $\phi_{*}(S):=(\phi \times \mathrm{id} \times \mathrm{id})(S)$ for a cobordism $S \subset F \times I \times I$.

Here Links $(M)$ stands for the category of oriented links in $M$ and oriented link cobordisms in $M \times I$. In this paper we are interested in the case of when $M$ is a solid torus, 
seen as a thickened annulus $\mathbb{A} \times I$ or as a thickened Möbius band $\mathbb{M} \widetilde{\times} I$. We write shortly $\mathscr{L} i n k s(\mathbb{A})$ and $\mathscr{L} i n k s(\mathbb{M})$ respectively for the categories of links. Both arise by considering tangles in a thickened plane with $\phi$ being the identity map or rotation by 180 degrees. Elements of $\mathscr{L} i n k s(\mathbb{A})$ are called annular links.

An analogous result holds for $\mathbf{C o b}$, the bicategory of flat tangles embedded in a stripe $\mathbb{R} \times I$, and for its famous quotient by local relations listed in Theorem 5.1, known as the Bar-Natan bicategory BN. Since a real line admits only two diffeomorphisms up to isotopy, the identification space in this case is either an annulus $\mathbb{A}$ or as a Möbius band $\mathbb{M}$. Hence, the horizontal traces of $\mathbf{B N}$ with the corresponding endofunctors recover the Bar-Natan categories $\mathscr{B} \mathcal{N}(\mathbb{A})$ and $\mathscr{B} \mathcal{N}(\mathbb{M})$. The identification $\mathscr{B} \mathcal{N}(\mathbb{A}) \cong h \operatorname{Tr}(\mathbf{B N})$ is also proven in [QR15].

Notice that 2-morphisms in $\operatorname{Tan}(F)$ are graded by the Euler characteristic, so that $\operatorname{Links}(M)$ can be quantized. Namely, we get the category

$$
\mathscr{L i n k} s_{q}(M):=\operatorname{hTr}_{q}\left(\mathbb{k} \operatorname{Tan}(F), \phi_{*}\right)
$$

where $\mathbb{k}$ is a fixed ring containing the value $q$, and $\mathbb{k} \operatorname{Tan}(F)$ is the linear extension of $\operatorname{Tan}(F)$, which 2-morphisms are formal linear combinations of cobordisms with coefficients from $\mathbb{k}$. This category it admits the following graphical description. Represent the image of $F \times\{0\}$ in $M$ as a cooriented membrane, with the coorientation induced from the orientation of $I$. The objects of $\mathscr{L} i n k s_{q}(M)$ are oriented links in $M$ that intersect the membrane transversely, whereas morphisms are link cobordisms up to isotopies, where an isotopy moving a cobordism of Euler characteristic $d$ through the membrane scales the cobordism by $q^{ \pm d}$ :
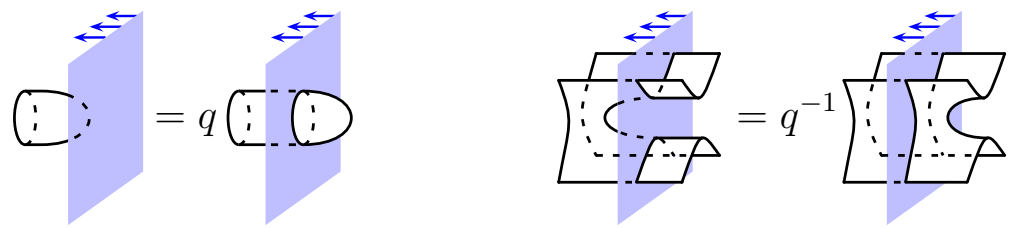

Because the local relations defining $\mathbf{B N}$ are homogeneous, we construct analogously the quantum annular Bar-Natan category as the additive closure of the quantum horizontal trace

$$
\mathscr{B} \mathcal{N}_{q}(\mathbb{A}):=\mathrm{hTr}_{q}^{\oplus}(\mathbf{B N}) .
$$

In important feature of $\mathscr{B} \mathcal{N}_{q}(\mathbb{A})$ is that a torus wrapped once along the annulus evaluates to $q+q^{-1}$ instead of 2 , see (6.5).

\section{Link Homologies via Traces}

Our next goal is to construct new functorial invariants of links in a solid torus and in a Möbius band. The first annular link homology theory was constructed by Asaeda, Przytycki, and Sikora [APS04], and it can be rephrased as applying the APS TQFT functor

$$
\mathscr{F}_{\mathbb{A}}: \mathscr{B} \mathcal{N}(\mathbb{A}) \longrightarrow \operatorname{Mod}(\mathbb{k})
$$

to the formal Khovanov bracket [BN05]. Grigsby, Licata, and Wehrli observed in [GLW15] that the annular link homology admits an action of $\mathfrak{s l}_{2}$ commuting with the maps induced by annular cobordisms. This motivates the search of a quantized annular homology, on which the quantum $\mathfrak{s l}_{2}$ acts. 
Our aim is to use $\mathscr{B} \mathcal{N}_{q}(\mathbb{A})$ to deform the APS construction. Even though it is immediate that a torus evaluates in $\mathscr{B N}_{q}(\mathbb{A})$ to $q+q^{-1}$ when it intersects the membrane in a circle, this does not extend naively to a TQFT functor. Instead, we use the whole algebraic machinery developed in the first part of the paper to construct such a TQFT, especially Theorem A, the universality of the horizontal trace, and the Lefschetz preshadow on the homotopy bicategory of Chen-Khovanov bimodules, induced by the quantum Hochschild homology.

For this purpose, let us examine the homology of tangles constructed by Chen and Khovanov. In [CK14] they defined a family of diagrammatic algebras $A^{n}$, commonly called arc algebras, associated a graded $\left(A^{m}, A^{n}\right)$-bimodule with each flat $(m, n)$-tangle, and constructed a bimodule map for any cobordism of flat $(m, n)$-tangles. This gives the bifunctor

$$
\mathbf{F}_{C K}: \mathbf{B N} \longrightarrow \text { gBirep }
$$

valued in the bicategory of sweet graded bimodules. A precomposition with the formal Khovanov bracket $\llbracket-\rrbracket$ results in

$$
\widetilde{\mathbf{F}}_{C K}: \operatorname{Tan} \longrightarrow C_{0 m}^{b}(\mathrm{gBirep}),
$$

which assigns to an $(m, n)$-tangle $T$ a chain complex $C_{C K}(T)$ of graded $\left(A^{m}, A^{n}\right)$-bimodules, the homotopy class of which is an isotopy invariant of $T$.

The algebra $A^{n}$ categorifies the $n$-th tensor power of the fundamental representation $V_{1}$ of $\boldsymbol{U}_{q}\left(\mathfrak{s l}_{2}\right)$. It was first introduced by Braden [Bra02] using generators and relations to describe the category of perverse sheaves on Grassmannians. The arc algebras and their representations were independently studied by Brundan and Stroppel [BS11, BS10].

We can now use our previous results to extend any given preshadow on gBirep to the homotopy category of complexes, and then pull it back along $\widetilde{\mathbf{F}}_{C K}$ to obtain a preshadow on Tan. Since all involved bicategories have duals, such a preshadow factors through $\mathrm{hTr}_{q}(\mathbf{T a n})$ defining an annular link homology theory.

An immediate choice of a preshadow on gBirep is the Hochschild homology $\mathrm{HH}_{\bullet}$ of Chen-Khovanov bimodules. However, we can also utilize the grading and use the quantum Hochschild homology $q H_{\bullet}$, a one parameter deformation of $H_{\bullet}$ that factors through the quantum horizontal trace of gBirep. This results in the following commuting diagram

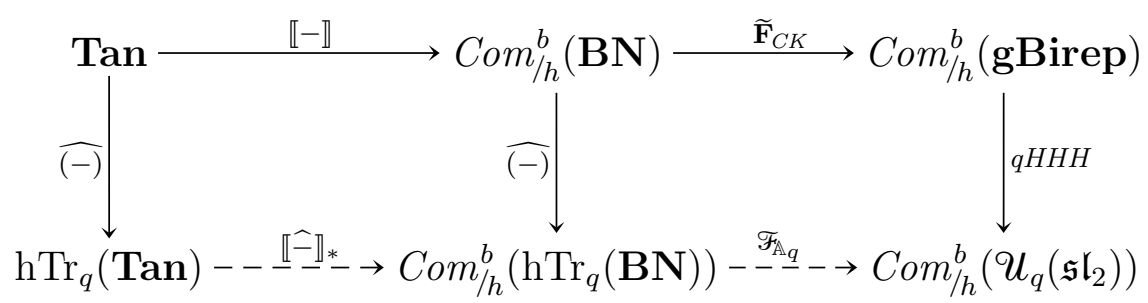

where the horizontal maps are functors and the vertical one are preshadows. The notation $q H H H$ means that we apply $q H H$ • to each bimodule in the Chen-Khovanov complex separately.

To identify the bottom right corner of this diagram we apply the $K$-theoretic invariance to arc algebras. We deduce that the Chern character map $K_{0}\left(A^{n}\right) \longrightarrow q H_{\bullet}\left(A^{n}\right)$ is actually an isomorphism. Hence, the higher Hochschild homology of $A^{n}$ vanishes and $q H H_{0}\left(A^{n}\right) \cong V_{1}^{\otimes n}$. This relates $\mathrm{hTr}_{q}^{\oplus}($ Birep $)=\mathscr{B} \mathcal{N}_{q}(\mathbb{A})$ with the graded representation category gRep $\left(\mathcal{U}_{q}\left(\mathfrak{s l}_{2}\right)\right)$. Finally, to construct our TQFT functor

$$
\mathscr{F}_{\mathbb{A}_{q}}: \mathscr{B} \mathcal{N}_{q}(\mathbb{A}) \longrightarrow \mathrm{g} \mathscr{R e p}\left(\boldsymbol{U}_{q}\left(\mathfrak{s l}_{2}\right)\right)
$$


we use the naturality of the Chern character map to identify $\mathscr{B} \mathcal{N}_{q}(\mathbb{A})$ with (the graded extension of) the Temperley-Lieb category $\mathscr{T}_{\mathscr{L}}$ and $\mathscr{F}_{\mathbb{A}_{q}}$ with the faithful functor from $\mathscr{T} \mathscr{L}$ to gRep $\left(\mathcal{U}_{q}\left(\mathfrak{s l}_{2}\right)\right)$.

This produces a well-defined homology for annular links. To any annular closure $L$ of an $(n, n)$-tangle $T$ it assigns a chain complex

$$
C K h_{\mathbb{A}_{q}}(L):=q H H H_{\bullet}\left(A^{n}, C_{C K}(T)\right)=q H H H_{0}\left(A^{n}, C_{C K}(T)\right)
$$

and to any annular cobordism an induced chain map. In addition to the homological and quantum grading, $C K h_{\mathbb{A}_{q}}(L)$ admits a third grading, called the annular grading, which comes from the weigh decomposition of the Chen-Khovanov invariant.

The chain maps induced by annular cobordisms are defined only up to multiplication by $\pm q^{ \pm 1}$ : the sign comes from the fact that $\widetilde{\mathbf{F}}_{C K}$ is merely a projective functor, and the overall power of $q$ is not well-defined, because it depends on the presentation of an annular cobordism as a horizontal closure of a surface with corners. We call this behavior ' $q$-projective'.

Theorem B. The quantum annular homology $K h_{\mathbb{A}_{q}}(L)$ is a triply graded invariant of an annular link $L$, which is q-projectively functorial with respect to annular link cobordisms. Moreover, it admits an action of the quantum group $\mathfrak{U}_{q}\left(\mathfrak{s l}_{2}\right)$ that commutes with the differential and the maps induced by annular link cobordisms intertwine this action.

It was conjectured by Auroux, Grigsby, and the third author in [AGW15] that the ASP homology of the braid closure coincides with the Hochschild homology of the ChenKhovanov complex associated to that braid. They checked this conjecture in the nextto-top annular grading.

Observe that the quantum annular homology arises as the second page of the spectral sequence associated to $q C H_{\bullet}\left(A^{n}, C_{C K}(T)\right)$, the bicomplex computing the quantum Hochschild complex for a complex of bimodules. Using the vanishing of the higher quantum Hochschild homology groups for arc algebras, we can actually show that this spectral sequence collapses at the second page. Hence we have the following.

Theorem C. Let $\widehat{T}$ be the annular closure of an $(n, n)$-tangle $T$. Then there is an isomorphism

$$
K h_{\mathbb{A}_{q}}(\widehat{T}) \cong q H_{\bullet}\left(A^{n} ; C_{C K}(T)\right),
$$

natural with respect to chain maps associated to tangle cobordisms. The annular grading in $K h_{\mathbb{A}}(\widehat{T})$ corresponds to the weight decomposition of $C_{C K}(T)$.

When $q=1$, Theorem $\mathrm{C}$ proves Conjecture 1.1 from [AGW15] and motivates us to call our new link invariant the quantum annular link homology.

We also show by explicit computation that the rank of the quantum annular link homology for $(2, n)$ torus links does depend on the quantum parameter $q$. Hence, the quantized theory is richer than APS annular link homology (which is the case $q=1$ ).

There are many papers devoted to fixing the sign issue to get a strictly functorial Khovanov homology [Vo15, Bla10, CMW09, Ca07]. In our next paper [BHPW18] we will reconstruct the Chen-Khovanov invariant using Blanchet foams to obtain a strictly functorial quantum annular link homology theory with an intertwining $\boldsymbol{u}_{q}\left(\mathfrak{g l}_{2}\right)$-action. 


\section{Applications and Generalizations}

Let us first argue why the quantum annular link homology is actually more sensible to 4D topology than APS.

A link cobordism $W: L \longrightarrow L$ induces a (projective) map $W_{*}: K h(L) \longrightarrow K h(L)$ on Khovanov homology of $L$. The trace class of this endomorphism is an invariant of the annular closure $\widehat{W}$ of $W$ and is characterised by its Lefschetz trace

$$
\Lambda\left(W_{*}\right)=\sum_{i, j}(-1)^{i} q^{j} \operatorname{tr} W_{*}^{i, j}
$$

where $W_{*}^{i, j}$ is the component of $W_{*}$ in homological grading $i$ and quantum grading $j$, and $t r$ is the Hattori-Stallings trace (which is the usual linear trace when we work over a field). We prove the following result.

Theorem D. Let $\widehat{W} \subset \mathbb{S}^{1} \times \mathbb{R}^{3}$ be a closed surface obtained as an annular closure of a link cobordism $W: L \longrightarrow L$ with $L \subset \mathbb{R}^{3}$. Then $K h_{\mathbb{A}_{q}}(\widehat{W})=\Lambda\left(W_{*}\right)$ is the graded Lefschetz trace of $W_{*}: K h(L) \longrightarrow K h(L)$, the endomorphism of the Khovanov homology of $L$. In particular, $K h_{\mathbb{A}_{q}}\left(\mathbb{S}^{1} \times L\right)$ coincides with the Jones polynomial $J(L)$.

Note that the APS invariant is trivial for closed surfaces. Therefore, our invariant is a nontrivial deformation.

Next, we establish a nontrivial braid group action on the quantum annular homology of cablings of a framed long knot. Consider a framed annular knot $K \subset \mathbb{A} \times I$. It defines an embedding $\nu_{K}: \mathbb{A} \times I \longrightarrow \mathbb{A} \times I$ with the tubular neighborhood of $K$ as its image, and hence induces a functor $K_{*}$ : Tan $\longrightarrow \operatorname{Links}(\mathbb{A})$ mapping oriented points $B \subset \mathbb{R}^{2}$ to a collection of circles $K^{B}:=\nu_{K}\left(\mathbb{S}^{1} \times B\right)$, and an oriented tangle $T \subset \mathbb{R}^{2} \times I$ to the oriented cobordism $K^{T}:=\nu_{K}\left(\mathbb{S}^{1} \times T\right)$ between these circles. Applying the quantum annular homology produces a map of homology

$$
K h_{\mathbb{A}_{q}}\left(K^{T}\right): K h_{\mathbb{A}_{q}}\left(K^{B}\right) \longrightarrow K h_{\mathbb{A}_{q}}\left(K^{B^{\prime}}\right)
$$

for any oriented tangle $T \in \operatorname{Tan}\left(B, B^{\prime}\right)$, defined up to an overall power of $q$ (we work in characteristic 2 here to avoid the sign issue). This gives rise to a $q$-projectively functorial action of $\mathscr{T} a n$, i.e.

$$
K h_{\mathbb{A}_{q}}\left(K^{T^{\prime} T}\right)=q^{k} K h_{\mathbb{A}_{q}}\left(K^{T^{\prime}}\right) \circ K h_{\mathbb{A}_{q}}\left(K^{T}\right),
$$

for any composable tangles $T, T^{\prime}$, and some $k \in \mathbb{Z}$. The action was first observed in [GLW15] in the non-quantized setting. We compute this action and show that it factors through the Jones skein relation.

Theorem E. Let $K$ be a framed annular link, considered as an object in Links . There is a functorial action of $\mathscr{T}$ an on the quantum annular homology of oriented cablings of $K$, that takes a tangle $T$ to the chain map $K h_{\mathbb{A}_{q}}\left(K^{T}\right)$, and which intertwines the action of $\mathcal{U}_{q}\left(\mathfrak{s l}_{2}\right)$. It factors through the Jones skein relation

$$
q^{2} K h_{\mathbb{A}_{q}}\left(K^{\nearrow^{\nwarrow}}\right)-q^{-2} K h_{\mathbb{A}_{q}}\left(K^{\mathrm{N}}\right)=\left(q-q^{-1}\right) K h_{\mathbb{A}_{q}}\left(K^{5 \nwarrow}\right)
$$

if $K$ intersects the membrane in one point. 
The condition on the characteristic of the ring of scalars can be dropped once a strictly functorial version of Chen-Khovanov is used. This will be address in our next paper [BHPW18].

Let us now discuss few modifications and generalizations of Theorem B. Recall that we have identified links in a thickened Möbius band with twisted closures of tangles. Therefore, one may hope to get a parallel quantization of the APS homology for links with diagrams on a Möbius band. Indeed, such a construction is described in Section 7.3. The basic algebraic ingredient is the twisted quantum Hochschild homology, defined for a pair of a graded algebra $A$ and its automorphism. Here, we consider the automorphism of arc algebras induced by the horizontal flip of diagrams. Again, setting $q=1$ recovers the original APS homology.

We have already observed that any preshadow applied to $C_{C K}(T)$ produces an annular link invariant. In particular, we can precompose an $(n, n)$-tangle $T$ with and $n$-cabling of a framed $(1,1)$-tangle, such as a sequence of full twists. Geometrically, these theories correspond to different embeddings of the annulus into $\mathbb{R}^{3}$. In the other generalization we again fix a $(1,1)$-tangle $T$ and we assign the quantum annular homology of either $\widehat{T}$ or its mirror image with an essential circle in an annulus. We then use the duality between the homology of a link and its mirror image to define the differential in the chain complex.

In a follow up [BHPW] we will define a quantum homology for colored annular links. A very surprising feature of this construction is that the Cooper-Krushkal infinite complex categorifying the $n$-th Jones-Wenzl idempotent becomes finite in the annular closure for a generic value of $q$, and it does coincide with the annular $n$-colored Khovanov complex.

\section{Strategy for the Proof of Theorem B}

To construct our new homology theory we follow the general recipe for Khovanov homology as described in [BN05]. There, a link $L$ in a thickened surface $F$ is assigned a formal complex $\llbracket L \rrbracket$ in the Bar-Natan skein category $\mathscr{B} \mathcal{N}(F)$, whose objects are non-intersecting curves in $F$ and morphisms are cobordisms in $F \times I$. To construct actual homology one then applies to $\llbracket L \rrbracket$ a certain TQFT functor $\mathscr{F}_{F}: \mathscr{B} \mathcal{N}(F) \longrightarrow \operatorname{Mod}(\mathbb{k})$, where $\mathbb{k}$ is a fixed ring of scalars. The main body of this paper is devoted to the construction of such a functor when $F=\mathbb{A}$. The theory of preshadows and horizontal traces on endobicategories is needed to guarantee the existence of the TQFT functor $\mathscr{F}_{\mathbb{A}_{q}}$ in (1.6).

Let us first explain why the two bottom arrows in (1.6) are dashed. Strictly speaking, a quantum preshadow on $\operatorname{Com}_{/ h}^{b}(\mathbf{B N})$ factorizes through $\mathrm{hTr}_{q}\left(\operatorname{Com}_{/ h}^{b}(\mathbf{B N})\right)$ rather than $\mathrm{Com}_{/ h}^{b}\left(\mathrm{~h} \operatorname{Tr}_{q}(\mathbf{B N})\right)$. Lemma 3.16 resolves this problem by showing that we actually can apply $q H H$. to each bimodule separately, rather than to the complex of bimodules, and deal with flat tangles rather than formal complexes of them. Hence, the pullback shadow $\left(\mathbf{F}_{C K}\right)^{*} q H H_{0}$ defined on $\mathbf{B N}$ factors through $\mathscr{B} \mathcal{N}_{q}(\mathbb{A})$, inducing a linear functor

$$
\mathscr{F}_{\mathbb{A}_{q}}: \mathscr{B} \mathcal{N}_{q}(\mathbb{A}) \longrightarrow \operatorname{Mod}(\mathbb{k})
$$

To identify the target of this functor with $\mathscr{R e p}\left(\mathcal{U}_{q}\left(\mathfrak{s l}_{2}\right)\right)$ we need to find an isomorphism

$$
V_{1}^{\otimes n} \cong q H H_{0}\left(A^{n}\right)
$$

where $A^{n}$ is the bimodule associated by $\mathbf{F}_{C K}$ with the trivial $(n, n)$-tangle, and $q H H_{0}\left(A^{n}\right)$ is the module assigned to $n$ parallel essential circles in $\mathbb{A}$. 
When $q=1$, the existence of the isomorphism (1.9) follows from the invariance result for Hochschild homology due to Keller [Ke98]: if $A$ is a finite dimensional algebra, $E \subset A$ is a separable subalgebra such that $A=E \oplus \operatorname{rad}(A)$, each simple $A$-module is onedimensional, and $A$ has finite global dimension, then $H_{\bullet}(E) \cong H_{\bullet}(A)$. It was proven by Brundan and Stroppel that arc algebras have finite global dimension when $\mathbb{k}$ is a field [BS11], and other conditions hold after setting $E=A_{0}^{n}$, the degree zero part of $A^{n}$. The latter consists of idempotents, and hence is isomorphic to $\mathbb{k}^{2^{n}}$. This provides the isomorphism (1.9) for $q=1$.

The 0th quantum Hochschild homology of $A^{n}$ can be computed by hands, which is all one needs to understand the construction of our invariant. However, computation of higher Hochschild homology is needed to identify the invariant with the total Hochschild homology of Chen-Khovanov bimodules, as conjectured in [AGW15]. For that we reprove the Keller's result for quantum and - more generally - for twisted Hochschild homology of an algebra $A$ by identifying the latter with quantum Hochschild-Mitchell homology of the category of finite dimensional representations of $A$, twisted by an appropriate endofunctor. The advantage of replacing algebras and bimodules with representation categories and functors is that the latter provides a more flexible framework, in which the action of bimodules on homology, and so the $K$-theoretic invariance, is easier to understand, see Section 4.3.

Further we observe that the canonical embedding of $\mathrm{vTr}_{q}(\mathbf{B N})$ into $h \operatorname{Tr}_{q}(\mathbf{B N})$ is an equivalence of categories. This implies that every cobordism in $h \operatorname{Tr}_{q}(\mathbf{B N})$ is a linear combination of those of the form $\mathbb{S}^{1} \times T$, where $T$ is a Temperley-Lieb diagram. Therefore, the isomorphism (1.9) determines $\mathscr{F}_{\mathbb{A}_{q}}$ completely, leading to a commuting diagram of functors

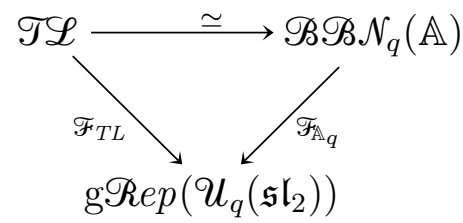

where the horizontal functor sends a flat tangle $T$ from $\mathscr{T} \mathscr{L}$ to the annular cobordism $\mathbb{S}^{1} \times T$, and $\mathscr{F}_{T L}$ is the Reshetikhin-Turaev realization of the Temperley-Lieb diagrams as $\mathcal{U}_{q}\left(\mathfrak{s l}_{2}\right)$ intertwiners between tensor powers of $V_{1}$, the fundamental representation of the quantum group. The category $\mathscr{B} \mathscr{B} \mathcal{N}_{q}(\mathbb{A})$ is a quotient of $\mathscr{B} \mathcal{N}_{q}(\mathbb{A})$ by a certain local relation defined in [Boe08].

It follows from that our annular quantum TQFTs functor $\mathscr{F}_{\mathbb{A}_{q}}$, when restricted to $\mathscr{B} \mathscr{B} \mathcal{N}_{q}(\mathbb{A})$, is faithful. Once the relation between $\mathscr{F}_{\mathbb{A}_{q}}$ and $\mathscr{F}_{T L}$ is established, we can directly see that setting $q=1$ recovers the APS TQFT functor.

\section{Outline}

The paper is organized as follows. In Section 2 we develop the theory of traces in endocategories. We discuss fundamental properties of the universal twisted trace, such as the universal property, functoriality, and connection to the additive Grothendieck group. The special cases of triangulated and graded endocategories are treated separately. The section ends with a number of examples.

Section 3 is devoted to the construction of categorical traces in endobicategories. We first introduce the notion a (pre)shadow and construct the twisted horizontal trace. Its universal property and functoriality are shown in Sections 3.2 and 3.3. Then we discuss 
the connection with the vertical trace and prove that the horizontal trace is a categorification of the universtal trace of an endocategory. Triangulated and quantum preshadows are discussed further. Again, all examples are gathered at the end of the section. Theorem A is proven in Section 3.8.7.

After the theory of categorified traces is established we take a closer look at Hochschild homology and its quantization in Section 4. We show that it is a preshadow on the category of small linear categories and prove its $K$-theoretic invariance.

With Section 5 we move to the topological part of the paper. Here we review link homology theories used in this paper: the formal bracket of Bar-Natan, the AsaedaPrzytycki-Sikora homology for links in a thickened annulus and in a thickened Möbius band, and the Chen-Khovanov homology for tangles.

We quantize the APS homology in Section 5. Theorems B and C are proven in Section 6.3, whereas 6.4 presents a computation of the invariant for torus links.

Section 7 contains further applications and generalizations: an extension to annular link cobordisms, the action of oriented tangles on cablings, quantization of the APS homology for links in a thickened Möbius band, and two generalizations of the annular homology.

Finally, we gathered in Appendix basic definitions and results concerning links and tangles, representations of $\boldsymbol{U}_{q}\left(\mathfrak{s l}_{2}\right)$, and elements of the (bi)category theory.

\section{Basic conventions and notation}

Throughout the paper $\mathbb{k}$ is a fixed unital commutative ring or field such as $\mathbb{Z}, \mathbb{Z}_{p}$, or $\mathbb{C}$, and and linearity means linearity over $\mathbb{k}$. An algebra means a $\mathbb{k}$-algebra that is projective over $\mathbb{k}$ and likewise for modules and bimodules.

Graded means always $\mathbb{Z}$-graded. We denote by $\{d\}$ the upwards degree shift, i.e. $M\{d\}_{i}=M_{i-d}$ for a graded module $M=\bigoplus_{d} M_{d}$.

A differential in a complex has homological degree +1 increases the homological degree. Thus we follow the cohomological notation and put indices as superscripts. The only exception is the Hochschild homology. The homological degree shift $[d]$ moves a complex downwards, i.e. $C[d]^{i}=C^{d+i}$.

Ordinary categories are typed with calligraphc letters $(\mathscr{C}, \mathscr{V}$ ect, etc.), whereas bold letters are reserved for bicategories (C, Rep, etc.). We usually use small latin letters for objects $(x, y$, etc.) and for morphisms $(f, g$, etc.), whereas 2 -morphisms are named by greek letters $(\alpha, \beta$, etc. $)$, with the exception of canonical isomorphisms in bicategories, for which gothic letters are used $(\mathfrak{a}, \mathfrak{l}, \mathfrak{m}$, etc.). Capital letters are mostly reserved for functors.

\section{Acknowledgements}

The authors are grateful to Adrien Brochier, Matthew Hogancamp, Mikhail Khovanov, Slava Krushkal, Aaron Lauda, David Rose, and Paul Wedrich for stimulating discussions. During an early stage of the research Robert Lipshitz suggested to look on higher Hochschild homology of the arc algebras and Ben Webster pointed a connection between Hochschild homology and the global dimension. The first two authors are supported by the NCCR SwissMAP founded by the Swiss National Science Foundation. The third author was supported by the NSF grant DMS-1111680. 


\section{Generalized traces}

We start with a discussion on twisted traces in general categories. Section 2.2 deals with traces on homotopy categories of complexes, whereas 2.3 describes how twisted traces can be deformed in case of graded categories. The section ends with a list of examples, the most important of which are the twisted Hattori-Stallings trace (2.4.6) and the annular closure of a tangle (2.4.8). For a brief list of constructions on categories see Appendix A.3.

\section{$2.1 \quad$ Twisted traces}

Choose a category $\mathscr{C}$ with an endofunctor $\Sigma: \mathscr{C} \longrightarrow \mathscr{C}$. The following definition extends the usual notion of a symmetric trace.

Definition 2.1. A collection $t=\left\{t_{x}\right\}_{x \in \mathscr{C}}$ of functions $t_{x}: \mathscr{C}(x, \Sigma x) \longrightarrow S$ valued in a set $S$ is a $\Sigma$-twisted trace or a trace on $\mathscr{C}$ with a monodromy $\Sigma$ if $t_{y}(\Sigma f \circ g)=t_{x}(g \circ f)$ for every pair of morphisms $\Sigma x \stackrel{g}{\longleftarrow} y \stackrel{f}{\longleftarrow} x$.

We shall sometimes refer to traces defined above as right traces, whereas a left trace is defined dually as a collection of morphisms from $\mathscr{C}(\Sigma x, x)$; the trace condition takes the form $t_{y}(f \circ \Sigma g)=t_{x}(g \circ f)$. The two definitions are clearly equivalent when $\Sigma$ is invertible. The naming convention is motivated by traces in pivotal categories, see Example 2.4.7.

Lemma 2.2. A trace $t$ with monodromy $\Sigma$ is $\Sigma$-invariant, i.e. $t_{\Sigma x}(\Sigma f)=t_{x}(f)$ for any $f \in \mathscr{C}(x, \Sigma x)$.

Proof. Take $y=\Sigma x$ and $g=\mathrm{id}_{\Sigma x}$ in the trace relation.

We often write $t:(\mathscr{C}, \Sigma) \longrightarrow S$ for a twisted trace on $\mathscr{C}$, despite that it is not defined for all morphisms. Further natural conditions on the components of twisted traces are imposed if $(\mathscr{C}, \Sigma)$ has an additional structure. For instance, when both $\mathscr{C}$ and $\Sigma$ are linear, then $S$ is assumed to be a module over the ring of coefficients $\mathbb{k}$ and each component $t_{x}: \mathscr{C}(x, \Sigma x) \longrightarrow S$ to be a linear homomorphism.

From the point of view of Category Theory, a twisted trace is a dinatural transformation from $\mathscr{C}(-, \Sigma(-))$ to the constant bifunctor $\Delta_{S}$ that assigns $S$ to any pair of objects and $\operatorname{id}_{S}$ to any pair of morphisms. It follows that there exists a universal $\Sigma$-twisted trace $\operatorname{tr}^{\Sigma}:(\mathscr{C}, \Sigma) \longrightarrow \operatorname{Tr}(\mathscr{C}, \Sigma)$ when $\mathscr{C}$ is small: the coend of $\mathscr{C}(-, \Sigma(-))$ [ML98, Chapter IX.6]. Explicitly,

$$
\operatorname{Tr}(\mathscr{C}, \Sigma):=\coprod_{x \in \operatorname{Ob}(\mathscr{C})} \mathscr{C}(x, \Sigma x) / \Sigma f \circ g \sim g \circ f
$$

or, when $\mathscr{C}$ is $\mathbb{k}$-linear,

$$
\operatorname{Tr}(\mathscr{C}, \Sigma):=\bigoplus_{x \in \mathrm{Ob}(\mathscr{C})} \mathscr{C}(x, \Sigma x) / \operatorname{span}_{\mathbb{k}}\{\Sigma f \circ g-g \circ f\}
$$

where $f$ and $g$ run through all pairs of morphisms $\Sigma x \stackrel{g}{\longleftarrow} y \stackrel{f}{\longleftarrow} x$ in $\mathscr{C}$. Each component of $\operatorname{tr}^{\Sigma}$ takes a morphism $f \in \mathscr{C}(x, \Sigma x)$ to its equivalence class $\operatorname{tr}^{\Sigma}(f) \in \operatorname{Tr}(\mathscr{C}, \Sigma)$, called the trace class of $f$. The universality means that every twisted trace $t:(\mathscr{C}, \Sigma) \longrightarrow S$ 
factorizes uniquely through $\operatorname{tr}^{\Sigma}$, i.e. there is a unique (linear) function $u$ : $\operatorname{Tr}(\mathscr{C}, \Sigma) \longrightarrow S$ such that the following triangle

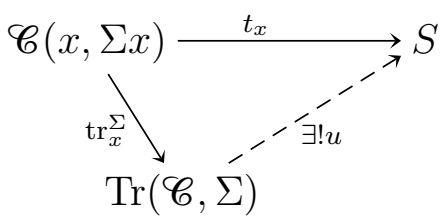

commutes for each object $x \in \mathscr{C}$. Explicitly, $u\left(\operatorname{tr}^{\Sigma}(f)\right)=t_{x}(f)$ for $f \in \mathscr{C}(x, \Sigma x)$.

We shall often refer to $\operatorname{Tr}(\mathscr{C}, \Sigma)$ as the $\Sigma$-twisted trace of $\mathscr{C}$, keeping in mind the components $\operatorname{tr}_{x}^{\Sigma}$. Moreover, following the standard convention, we omit $\Sigma$ from the notation if it is the identity functor, writing simply $\operatorname{Tr}(\mathscr{C})$.

The trace $\operatorname{Tr}(\mathscr{C}, \Sigma)$ can be seen as a coend parametrized by $\Sigma$. Therefore, by the Parameter Theorem for coends [ML98, cp. Theorem IX.7.2], a natural transformation of endofunctors induces a map between traces. In fact, such a map exists in a more general situation when $\mathscr{C}$ can vary as well. First let us create the appropriate framework.

Definition 2.3. An endocategory is a pair $(\mathscr{C}, \Sigma)$ consisting of a category $\mathscr{C}$ and a functor $\Sigma: \mathscr{C} \longrightarrow \mathscr{C}$. Functors between endocategories $(\mathscr{C}, \Sigma)$ and $\left(\mathscr{C}^{\prime}, \Sigma^{\prime}\right)$ are pairs $(F, \omega)$ consisting of a functor $F: \mathscr{C} \longrightarrow \mathscr{C}^{\prime}$ and a natural transformation $\omega: F \Sigma \longrightarrow \Sigma^{\prime} F$. It is finally understood that a natural transformation $\eta:(F, \omega) \longrightarrow\left(F^{\prime}, \omega^{\prime}\right)$ between two such functors intertwines $\omega$ with $\omega^{\prime}$.

The above describes $\mathscr{E}$ ndo $\mathscr{C} a t$, the 2-category of endocategories. The condition for a natural transformation $\eta$ can be rephrased by saying that the square

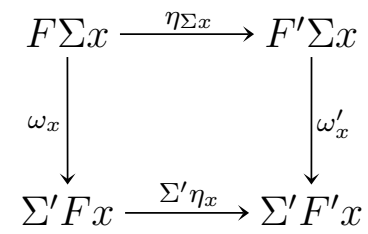

commutes for every $x \in \mathscr{C}$. In the following we argue that isomorphism classes of functors between endocategories induce maps between traces.

Choose a functor $(F, \omega):(\mathscr{C}, \Sigma) \longrightarrow\left(\mathscr{C}^{\prime}, \Sigma^{\prime}\right)$ and a trace $t:\left(\mathscr{C}^{\prime}, \Sigma^{\prime}\right) \longrightarrow S$. We check directly that $F^{*} t_{x}(f):=t_{F x}\left(\omega_{x} \circ F f\right)$ defines a $\Sigma$-twisted trace on $\mathscr{C}$; we call it the pullback of $t$ along $F$.

Proposition 2.4. Choose a trace $t:\left(\mathscr{C}^{\prime}, \Sigma^{\prime}\right) \longrightarrow S$. Then $(G F)^{*} t=F^{*}\left(G^{*} t\right)$ for a pair of functors $(F, \omega):(\mathscr{C}, \Sigma) \longrightarrow\left(\mathscr{C}^{\prime}, \Sigma^{\prime}\right)$ and $(G, \omega):\left(\mathscr{C}^{\prime}, \Sigma^{\prime}\right) \longrightarrow\left(\mathscr{C}^{\prime \prime}, \Sigma^{\prime \prime}\right)$. Morevoer, $F^{*} t=F^{* *} t$ if $(F, \omega)$ and $\left(F^{\prime}, \omega^{\prime}\right)$ are isomorphic functors.

Proof. The first equality follows from a direct computation

$$
F^{*}\left(G^{*} t\right)_{x}(f)=\left(G^{*} t\right)_{F x}\left(\omega_{x} \circ F f\right)=t_{G F x}\left(\omega_{F x}^{\prime} \circ G \omega_{x} \circ G F f\right)=(G F)^{*} t_{x}(f),
$$

while for the second statement we use (2.4) to compute

$$
\Sigma^{\prime} \eta_{x} \circ \omega_{x} \circ F f \circ \eta_{x}^{-1}=\omega_{x}^{\prime} \circ \eta_{\Sigma x} \circ F f \circ \eta_{x}^{-1}=\omega_{x}^{\prime} \circ F^{\prime} f
$$

where $\eta:(F, \omega) \longrightarrow\left(F^{\prime}, \omega^{\prime}\right)$ is a natural isomorphism.

Corollary 2.5. A trace $t$ on $(\mathscr{C}, \Sigma)$ can be twisted by a natural transformation $\eta: \Sigma \longrightarrow \Sigma$ to $\left(\eta^{*} t\right)_{x}:=t_{x}\left(\eta_{x} \circ f\right)$. 
Proof. The trace $\eta^{*} t$ is a pullback of $t$ along the functor of endocategories (Id, $\left.\eta\right)$.

Let $\mathscr{E} n d o \mathscr{C} a t^{\leqslant 1}$ be the truncation of $\mathscr{E} n d o \mathscr{C}$ at to a category obtained by forgetting natural transformation and identifying isomorphic functors. Proposition 2.4, when combined with the universal property of Tr, implies functoriality of the universal trace.

Corollary 2.6. There is a functor $\operatorname{Tr}: \mathscr{E}$ ndo $\mathscr{C} a t^{\leqslant 1} \longrightarrow$ Set that sends an endocategory $(\mathscr{C}, \Sigma)$ to its universal trace $\operatorname{Tr}(\mathscr{C}, \Sigma)$ and a functor $(F, \omega):(\mathscr{C}, \Sigma) \longrightarrow\left(\mathscr{C}^{\prime}, \Sigma^{\prime}\right)$ to the map $\operatorname{Tr}(F, \omega): \operatorname{Tr}(\mathscr{C}, \Sigma) \longrightarrow \operatorname{Tr}\left(\mathscr{C}^{\prime}, \Sigma^{\prime}\right)$ that takes $\operatorname{tr}^{\Sigma}(f)$ into $\operatorname{tr}^{\Sigma^{\prime}}(\omega \circ F f)$.

Recall that the split Grothendieck group $K_{0}(\mathscr{C})$ of an additive category is generated by isomorphism classes $[x]$ of objects of $\mathscr{C}$ modulo the relation $[x \oplus y]=[x]+[y]$. There is a natural additive map $h: K_{0}(\mathscr{C}) \longrightarrow \operatorname{Tr}(\mathscr{C})$ called the Chern character [BHLZ14] that takes $[x]$ to the trace class of $\mathrm{id}_{x}$. This map is no longer well-defined for twisted traces - identity morphisms does not represent trace classes in $\operatorname{Tr}(\mathscr{C}, \Sigma)$. However, the map can be 'twisted' in certain cases.

Theorem 2.7. Let $(\mathscr{C}, \Sigma)$ be an additive endocategory and $\eta:$ Id $\longrightarrow \Sigma$ a natural transformation of functors. Then there is a natural homomorphism of abelian groups

$$
h^{\eta}: K_{0}^{s p}(\mathscr{C}) \longrightarrow \operatorname{Tr}(\mathscr{C}, \Sigma), \quad[x] \cong \longmapsto \operatorname{tr}^{\Sigma}\left(\eta_{x}\right),
$$

called the twisted Chern character.

Proof. By Corollary 2.6 there is a map of traces $\eta_{*}: \operatorname{Tr}(\mathscr{C}) \longrightarrow \operatorname{Tr}(\mathscr{C}, \Sigma)$, which takes the trace class $\operatorname{tr}(f)$ of $f \in \mathscr{C}(x, x)$ into $\operatorname{tr}^{\Sigma}\left(\eta_{x} \circ f\right)$. Compose it with the usual Chern character to obtain $h^{\eta}$.

Being defined for not necessarily additive categories is a big advantage of traces when compared to the Grothendieck group. In fact, $\operatorname{Tr}(\mathscr{C}, \Sigma)$ can be often computed by considering only a certain subset of objects, which may not be preserved by $\Sigma$. For a precise statement, define the trace of $\mathscr{C}$ restricted to $B \subset \mathrm{Ob}(\mathscr{C})$ as the quotient

$$
\operatorname{Tr}(\mathscr{C} \mid B, \Sigma):=\bigoplus_{x \in B} \mathscr{C}(x, \Sigma x) / \operatorname{span}_{\mathbb{k}}\{\Sigma f \circ g-g \circ f\}
$$

where $f$ and $g$ run through all pairs of morphisms $\Sigma x \stackrel{g}{\longleftarrow} y \stackrel{f}{\longleftarrow} x$ in $\mathscr{C}$ with $x, y \in B$ (but $\Sigma x$ may not belong to $B$ ). There is a natural homomorphism

$$
\operatorname{Tr}(\mathscr{C} \mid B, \Sigma) \longrightarrow \operatorname{Tr}(\mathscr{C}, \Sigma)
$$

We say that $B$ generates additively $\mathscr{C}$ if every object of $\mathscr{C}$ is a direct summand of an object of the form $b_{1} \oplus \cdots \oplus b_{r}$ with $b_{i} \in B$. For example, $\mathrm{Ob}(\mathscr{C})$ generates additively both the additive closure $\mathscr{C}^{\oplus}$ and the idempotent completion $\operatorname{Kar}(\mathscr{C})$ of $\mathscr{C}$ (see Section A.3). Both categories come with natural endofunctors induced by $\Sigma$, for which we use the same symbol.

Proposition 2.8. Suppose that $\mathscr{C}$ is generated additively by B. Then (2.9) is an isomorphism. In particular, the inclusions of endocategories

$$
\begin{aligned}
& (\mathscr{C}, \Sigma) \longrightarrow\left(\mathscr{C}^{\oplus}, \Sigma\right), \quad x \longmapsto(x) \\
& (\mathscr{C}, \Sigma) \longrightarrow(\operatorname{Kar}(\mathscr{C}), \Sigma), \quad x \longmapsto \operatorname{id}_{x}
\end{aligned}
$$

induce natural isomorphisms of the universal traces. 
This is a generalization of [BHLZ14, Sections 3.4, 3.5] and a special case of Proposition 4.2. We leave the proof as an easy exercise.

Remark 2.9. Although we discuss here only right traces, all the results can be easily translated to left traces. In particular, an endocategory $(\mathscr{C}, \Sigma)$ admits the universal left trace $\operatorname{Tr}^{\ell}(\mathscr{C}, \Sigma)$. Unless stated otherwise, the universal trace always means the right trace.

\subsection{Triangulated traces}

Let $\mathscr{T}$ be a triangulated category and $\Sigma$ its triangulated endofunctor, meaning that there is a natural isomorphism $\alpha_{x}: \Sigma(X[1]) \stackrel{\cong}{\longrightarrow}(\Sigma X)[1]$ and for each distinguished triangle

$$
X \stackrel{f}{\longrightarrow} Y \stackrel{g}{\longrightarrow} Z \stackrel{h}{\longrightarrow} X[1]
$$

the triangle

$$
\Sigma X \stackrel{\Sigma f}{\longrightarrow} \Sigma Y \stackrel{\Sigma g}{\longrightarrow} \Sigma Z \stackrel{\alpha_{X} \circ \Sigma h}{\longrightarrow} \Sigma X[1]
$$

is also distinguished. We say that a trace $t$ on $(\mathscr{T}, \Sigma)$ is triangulated if it is additive with respect to homomorphisms of distinguished triangles, i.e.

$$
t(b)=t(a)+f(c)
$$

for any commutative diagram

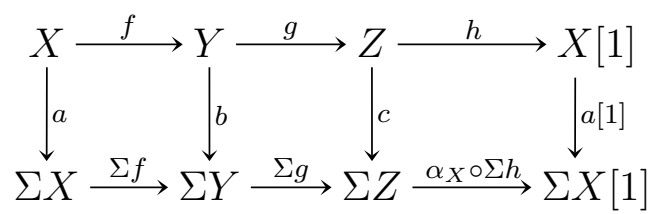

with distinguished triangles as rows.

Lemma 2.10. Assume that $t$ is a triangulated trace on $(\mathcal{T}, \Sigma)$. Then $t(f[1])=-t(f)$ for any $f \in \mathscr{T}(x, \Sigma x)$.

Proof. Apply the additivity of $t$ to the diagram $(2.15)$ with $X \longrightarrow 0 \longrightarrow X[1] \stackrel{- \text { id }}{\longrightarrow} X[1]$ as the top triangle and $(a, b, c)=(f, 0, f[1])$.

Likewise for usual traces, triangulated traces are stable under pullbacks along triangulated functors, and there is the universal triangulated trace $\operatorname{Tr}^{\triangle}(\mathscr{T}, \Sigma)$, through which all triangulated $\Sigma$-twisted traces on $\mathscr{T}$ factor.

A particular example of a triangulated category is $\operatorname{Com}_{/ h}^{b}(\mathscr{C})$, the homotopy category of bounded formal complexes over $\mathscr{C}$, i.e. sequences of objects and morphisms

$$
\ldots \longrightarrow C^{i} \stackrel{d^{i}}{\longrightarrow} C^{i+1} \stackrel{d^{i+1}}{\longrightarrow} C^{i+2} \longrightarrow \ldots
$$

with $d^{i+1} \circ d^{i}=0$ and $C^{i}=0$ except finitely many indices. The degree shift functor shifts the complex leftwards and negates its differential, whereas the distinguished triangles are of the form

$$
C^{\bullet} \stackrel{f^{\bullet}}{\longrightarrow} D^{\bullet} \stackrel{i n^{\bullet}}{\longrightarrow} \operatorname{cone}^{\bullet}(f) \stackrel{p r^{\bullet}}{\longrightarrow} C[1]^{\bullet},
$$

where cone $\bullet(f)$ stands for the mapping cone of $f$, see Section A.3 for the details. It is implicitly assumed that $\mathscr{C}$ is additive (if not, take its additive closure). 
Definition 2.11. A Lefschetz trace $\Lambda^{t}$ on $\operatorname{Com}_{/ h}^{b}(\mathscr{C})$ induced by a trace $t$ on $\mathscr{C}$ is defined by the formula

$$
\Lambda_{x}^{t}\left(f^{\bullet}\right):=\sum_{i}(-1)^{i} t_{x}\left(f^{i}\right)
$$

It is immediate that a Lefschetz trace satisfies the $\Sigma$-twisted trace relation if it is induced by a $\Sigma$-twisted trace. A little longer computation shows that it is triangulated. In fact, as explained below, all triangulated traces on $\operatorname{Com}_{/ h}^{b}(\mathscr{C})$ are Lefschetz traces. In what follows we consider $\mathscr{C}$ as a subcategory of $\mathrm{Com}_{/ h}^{b}(\mathscr{C})$ by understanding an object $X$ as the complex $(0 \longrightarrow X \longrightarrow 0)$ with $X$ at homological degree 0 .

Proposition 2.12. Every triangulated $\Sigma$-twisted trace on $\mathrm{Com}_{/ h}^{b}(\mathscr{C})$ is a Lefschetz trace, induced by its restriction to $\mathscr{C}$. In particular, $\operatorname{Tr}(\mathscr{C}, \Sigma) \cong \operatorname{Tr}^{\triangle}\left(\operatorname{Com}_{/ h}^{b}(\mathscr{C}), \Sigma\right)$.

Proof. Observe that a bounded complex $C^{\bullet}$ is a mapping cone of a map between smaller complexes: $\left(0 \longrightarrow C^{\text {min }} \longrightarrow 0\right)$, supported in homological degree $\min =\min \left\{i: C^{i} \neq 0\right\}$, and $C^{>\min }=\left(0 \longrightarrow C^{\min +1} \longrightarrow C^{\min +2} \longrightarrow \ldots\right)$. The first statement follows thus from the induction on the length of a complex together with additivity of triangulated traces (2.14). In particular, the universal triangulated $\Sigma$-twisted trace on $\mathrm{Com}_{/ h}^{b}(\mathscr{C})$ is the Lefschetz trace induced by the universal $\Sigma$-twisted trace on $\mathscr{C}$, which implies the second statement.

\subsection{Deformation of twisted traces}

A linear category $\mathscr{C}$ is graded if it admits an autoequivalence $\{1\}: \mathscr{C} \longrightarrow \mathscr{C}$, called the degree shift. The Grothendieck group $K_{0}^{s p}(\mathscr{C})$ of a graded category has a natural structure of a $\mathbb{Z}\left[q^{ \pm 1}\right]$-module by setting $q \cdot[x]:=[x\{1\}]$. Motivated by Theorem 2.7 we want to introduce a similar relation on the trace. There are two ways to do it: by defining the action of $q$ explicitly or by deforming the trace relation. In what follows we explore both approaches.

A graded endocategory is a quadruple $(\mathscr{C}, \Sigma,\{1\}, \alpha)$ consisting of a category $\mathscr{C}$, endofunctors $\Sigma$ and $\{1\}$, where the latter is an equivalence, and a natural isomorphism $\alpha: \Sigma(-)\{1\} \stackrel{\cong}{\longrightarrow} \Sigma(-\{1\})$. The equivalence $\{1\}$ is called the degree shift functor. We use it to equip the universal trace $\operatorname{Tr}(\mathscr{C}, \Sigma)$ with an action of $\mathbb{Z}\left[q^{ \pm 1}\right]$ by setting

$$
q \cdot \operatorname{tr}^{\Sigma}(f):=\operatorname{tr}^{\Sigma}\left(\alpha_{x} \circ f\{1\}\right)
$$

for any $f \in \mathscr{C}(x, \Sigma x)$. The following is an immediate consequence of this definition.

Corollary 2.13. Let $(\mathscr{C}, \Sigma,\{1\}, \alpha)$ be a graded additive endocategory and $\eta:$ Id $\longrightarrow \Sigma$ a natural transformation satisfying $\eta_{x\{1\}}=\alpha_{x} \circ \eta_{x}\{1\}$. Then the twisted Chern character $h^{\eta}: K_{0}^{s p}(\mathscr{C}) \longrightarrow \operatorname{Tr}(\mathscr{C}, \Sigma)$ is $\mathbb{Z}\left[q^{ \pm 1}\right]$-linear.

We shall now introduce the action differently by deforming the trace relation. For that recall the notion of a pregraded category: it is a category $\mathscr{C}$, morphism sets of which are graded $\mathbb{k}$-modules

$$
\mathscr{C}(x, y)=\bigoplus_{d \in \mathbb{Z}} \mathscr{C}(x, y)^{d}
$$

and the degree is additive with respect to the composition. Naturally, we say that $(\mathscr{C}, \Sigma)$ is a pregraded category when $\Sigma$ preserves the grading, i.e. $\Sigma\left(\mathscr{C}(x, y)^{d}\right) \subset \mathscr{C}(\Sigma x, \Sigma y)^{d}$ for all objects $x$ and $y$. 
A pregraded endocategory $(\mathscr{C}, \Sigma)$ can be transformed into a graded one $\left(\mathscr{C}^{g r}, \Sigma^{g r}\right)$ by introducing a formal degree shift and taking degree zero morphisms only. Thence, objects of $\mathscr{C}^{g r}$ are symbols $x\{d\}$ with $x \in \mathrm{Ob}(\mathscr{C})$ and $d \in \mathbb{Z}$, morphisms sets are $\mathscr{C}^{g r}(x\{a\}, y\{b\}):=\mathscr{C}(x, y)^{a-b}$, and the degree shift functor increases the number in brackets by one. The endofunctor $\Sigma^{g r}$ takes $x\{a\}$ to $(\Sigma x)\{a\}$.

Dually, a graded endocategory $(\mathscr{C}, \Sigma)$ can be extended to a pregraded $\left(\mathscr{C}^{\text {pre }}, \Sigma^{\text {pre }}\right)$ by forgetting the degree shift functor, while introducing morphisms of nonzero degrees by setting $\mathscr{C}^{\text {pre }}(x, y)^{d}:=\mathscr{C}(x\{d\}, y)$ and extending the composition with the formula

$$
\left(z \stackrel{g}{\longleftarrow} y\left\{d^{\prime}\right\}\right) \circ(y \stackrel{f}{\longleftarrow} x\{d\}):=\left(z \stackrel{g}{\longleftarrow} y\left\{d^{\prime}\right\} \stackrel{f\left\{d^{\prime}\right\}}{\longleftarrow} x\left\{d+d^{\prime}\right\}\right)
$$

To define $\Sigma^{\text {pre }}$ we use the natural isomorphism $\alpha$ :

$$
\begin{array}{ll}
\Sigma^{\text {pre }} x:=\Sigma x & \text { for } x \in \mathrm{Ob}\left(\mathscr{C}^{\text {pre }}\right)=\mathrm{Ob}(\mathscr{C}) \\
\Sigma^{\text {pre }} f:=\Sigma f \circ \alpha_{x}^{d} & \text { for } f \in \mathscr{C}^{\text {pre }}(x, y)^{d}=\mathscr{C}(x\{d\}, y)
\end{array}
$$

The two construction described above are clearly inverse to each other.

Definition 2.14. Choose a $\mathbb{k}\left[q^{ \pm 1}\right]$-module $S$. A quantum trace on a pregraded endocategory $(\mathscr{C}, \Sigma)$ is a linear trace $t:(\mathscr{C}, \Sigma) \longrightarrow S$ satisfying the deformed trace condition $t_{y}(\Sigma f \circ g)=q^{|f|} t_{x}(g \circ f)$ for every pair of homogeneous morphisms $\Sigma x \stackrel{g}{\longleftarrow} y \stackrel{f}{\longleftarrow} x$, where we write $|f|$ for the degree of $f$.

Write $\mathscr{C}_{q}$ for the category $\mathscr{C}$ with coefficients extended to $\mathbb{k}\left[q^{ \pm 1}\right]$. There exists a universal $\Sigma$-twisted quantum trace

$$
\operatorname{Tr}_{q}(\mathscr{C}, \Sigma):=\bigoplus_{x \in \mathrm{Ob}(\mathscr{C})} \mathscr{C}_{q}(x, \Sigma x) / \operatorname{span}_{\mathbb{k}\left[q^{ \pm 1}\right]}\left\{\Sigma f \circ g-q^{|f|} g \circ f\right\}
$$

with the obvious components $\operatorname{tr}_{q}^{\Sigma}: \mathscr{C}(x, \Sigma x) \longrightarrow \operatorname{Tr}_{q}(\mathscr{C}, \Sigma)$, through which every quantum trace on $(\mathscr{C}, \Sigma)$ factorizes. It is a graded $\mathbb{k}\left[q^{ \pm 1}\right]$-module, because the defining relation is homogeneous. One can see it as a one parameter deformation of $\operatorname{Tr}(\mathscr{C}, \Sigma)$. As before, we shall write $\operatorname{Tr}_{q}(\mathscr{C})$ when $\Sigma$ is the identity functor.

Quantum traces arise from a deformation of the endofunctor $\Sigma$. Indeed, a quantum trace on $(\mathscr{C}, \Sigma)$ is precisely a trace on $\left(\mathscr{C}_{q}, \Sigma_{q}\right)$ as defined in the previous section, where $\Sigma_{q} f:=q^{-|f|} \Sigma f$ for a homogeneous morphism $f$. In particular,

$$
\operatorname{Tr}_{q}(\mathscr{C}, \Sigma) \cong \operatorname{Tr}\left(\mathscr{C}_{q}, \Sigma_{q}\right)
$$

so that all the properties of traces can be easily translated to the quantum setting.

Proposition 2.15. A functor of pregraded endocategories $(F, \omega):(\mathscr{C}, \Sigma) \longrightarrow\left(\mathscr{C}^{\prime}, \Sigma^{\prime}\right)$ induces a map of universal quantum traces $\operatorname{Tr}_{q}(F, \omega): \operatorname{Tr}_{q}(\mathscr{C}, \Sigma) \longrightarrow \operatorname{Tr}_{q}\left(\mathscr{C}^{\prime}, \Sigma^{\prime}\right)$, which takes $\operatorname{tr}_{q}^{\Sigma}(f)$ into $\operatorname{tr}_{q}^{\Sigma^{\prime}}(\omega \circ F f)$.

Write as before $\operatorname{Tr}_{q}(\mathscr{C} \mid B, \Sigma)$ for the quantum trace restricted to $B \subset \mathrm{Ob}(\mathscr{C})$. We say that $\mathscr{C}$ is generated additively by $B$ in the graded sense if it is generated additively by $\tilde{B}:=\{x\{d\} \mid x \in B, d \in \mathbb{Z}\}$. The following is a special case of Proposition 4.2.

Proposition 2.16. The map $\operatorname{Tr}_{q}(\mathscr{C} \mid B, \Sigma) \longrightarrow \operatorname{Tr}_{q}(\mathscr{C}, \Sigma)$ is an isomorphism if $\mathscr{C}$ is generated additively by $B$ in a graded sense. In particular, $\operatorname{Tr}_{q}(\mathscr{C}, \Sigma) \cong \operatorname{Tr}_{q}\left(\mathscr{C}^{\oplus}, \Sigma\right)$ and $\operatorname{Tr}_{q}(\mathscr{C}, \Sigma) \cong \operatorname{Tr}_{q}(\operatorname{Kar}(\mathscr{C}), \Sigma)$. 
Because $\Sigma$ preserves the degree of morphisms, the universal quantum traces if a graded module. Its degree $d$ component

$$
\operatorname{Tr}_{q}^{d}(\mathscr{C}, \Sigma):=\bigoplus_{x \in \mathrm{Ob}(\mathscr{C})} \mathscr{C}_{q}(x, \Sigma x)^{d} / \operatorname{span}_{\mathbb{k}\left[q^{ \pm 1}\right]}\left\{\Sigma f \circ g-q^{|f|} g \circ f\right\}
$$

is annihilated by $\left(1-q^{d}\right)$, which follows immediately from Lemma 2.2.

Traces on graded endocategories and quantum traces on pregraded endocategories are closely related to each other.

Proposition 2.17. Let $(\mathscr{C}, \Sigma)$ be a pregraded extension of a graded endocategory $\left(\mathscr{C}^{0}, \Sigma^{0}\right)$. Then $\operatorname{Tr}\left(\mathscr{C}^{0}, \Sigma^{0}\right)$ and $\operatorname{Tr}_{q}^{0}(\mathscr{C}, \Sigma)$ are isomorphic $\mathbb{k}\left[q^{ \pm 1}\right]$-modules.

Proof. As the notation suggest, $\mathscr{C}^{0}\left(x, \Sigma^{0} x\right)$ are precisely the degree zero morphisms in $\mathscr{C}(x, \Sigma x)$. Therefore, there is an obvious surjective map $i: \operatorname{Tr}\left(\mathscr{C}^{0}, \Sigma^{0}\right) \longrightarrow \operatorname{Tr}_{q}^{0}(\mathscr{C}, \Sigma)$. We check first that it intertwines the action of $\mathbb{k}\left[q^{ \pm 1}\right]$. For that let $\iota_{x} \in \mathscr{C}(x, x\{1\})^{1}$ correspond to $\operatorname{id}_{x\{1\}} \in \mathscr{C}^{0}(x\{1\}, x\{1\})$ and notice the equality $f\{1\}=\iota_{\Sigma x} \circ f \circ \iota_{x}^{-1}$ for any morphism $f: x \longrightarrow \Sigma x$. Hence,

$$
\operatorname{tr}_{q}\left(\alpha_{x} \circ f\{1\}\right)=\operatorname{tr}_{q}\left(\alpha_{x} \circ \iota_{\Sigma x} \circ f \circ \iota_{x}^{-1}\right)=q \cdot \operatorname{tr}_{q}\left(\Sigma \iota_{x}^{-1} \circ \alpha_{x} \circ \iota_{\Sigma x} \circ f\right)=q \cdot \operatorname{tr}_{q}(f)
$$

in $\operatorname{Tr}_{q}^{0}(\mathscr{C}, \Sigma)$. To show that $i$ is injective, and hence an isomorphism, take any morphisms $f \in \mathscr{C}(x, y)^{d}$ and $g \in \mathscr{C}(y, \Sigma x)^{-d}$, and recall how $\Sigma$ and the composition of morphisms of nonzero degree is defined in $\mathscr{C}$ :

$$
\begin{aligned}
g \circ f & :=g \circ f\{-d\} \\
\Sigma f \circ g & :=\Sigma^{0} f \circ \alpha^{d} \circ g\{d\}
\end{aligned}
$$

where the left and right sides use the composition in $\mathscr{C}$ and $\mathscr{C}^{0}$ respectively. Therefore,

$$
\operatorname{tr}\left(\Sigma^{0} f \circ \alpha^{d} \circ g\{d\}\right)=\operatorname{tr}\left(\alpha^{d} \circ(g \circ f\{-d\})\{d\}\right)=q^{d} \operatorname{tr}(g \circ f\{-d\})
$$

in $\operatorname{Tr}\left(\mathscr{C}^{0}, \Sigma^{0}\right)$, revealing that the quantum trace relation in $\operatorname{Tr}_{q}^{0}(\mathscr{C}, \Sigma)$ is a consequence of the module structure on $\operatorname{Tr}\left(\mathscr{C}^{0}, \Sigma^{0}\right)$. In particular, the kernel of $i$ is trivial.

Remark 2.18. Triangulated traces also admit deformations as long as the triangulated category, on which they are defined, has an additional grading compatible with the triangulated structure. An example is the homotopy category $\mathrm{Com}_{/ h}(\mathscr{C})$ of a pregraded category $\mathscr{C}$, objects of which are formal complexes with a graded differential, and formal chain maps have graded components.

\subsection{Examples}

In what follows we provide a number of (twisted) traces that appear in algebraic or topological contexts. Of particular interest to us are the Hattori-Stallings trace (together with its twisted version) and annular closures of tangles. 


\subsubsection{The trace of a linear map}

Let $\mathscr{V}$ ect be the category of finite dimensional vector spaces over a field $\mathbb{F}$. It is a classical result from Linear Algebra that there is a unique collection of $\operatorname{maps} \operatorname{tr}_{V}: \operatorname{End}(V) \longrightarrow \mathbb{F}$ satisfying the trace relation and $\operatorname{such}$ that $\operatorname{tr}_{V}(\mathrm{id})=\operatorname{dim} V$. Explicitly,

$$
\operatorname{tr}(f):=\sum_{i} f_{i i} \in \mathbb{F}
$$

where $\left(f_{i j}\right)$ is a matrix representation of $f$ in some basis of $V$. This is the universal trace: $\mathscr{V}$ ect is generated additively by $B=\{\mathbb{F}\}$, so that $\operatorname{Tr}(\mathscr{V}$ ect $) \cong \operatorname{Tr}(\mathscr{V} e c t \mid\{\mathbb{F}\})=\mathbb{F}$.

\subsubsection{The graded trace}

In case of $\mathbb{Z}$-graded vector spaces the above trace admits a deformation

$$
\operatorname{tr}_{q}(f):=\sum_{n} q^{n} \operatorname{tr}\left(\left.f\right|_{V_{n}}\right) \in \mathbb{F}\left[q^{ \pm 1}\right]
$$

where $V_{n} \subset V$ is the subspace generated by homogeneous vectors of degree $n$. It satisfies the quantum trace relation:

$$
q^{|f|} \operatorname{tr}_{q}(g \circ f)=\sum_{n \in \mathbb{Z}} q^{n+|f|} \operatorname{tr}\left(\left.g \circ f\right|_{V_{n}}\right)=\sum_{n \in \mathbb{Z}} q^{n+|f|} \operatorname{tr}\left(\left.f \circ g\right|_{W_{n+|f|}}\right)=\operatorname{tr}_{q}(f \circ g)
$$

for any pair of homogeneous maps $V \stackrel{g}{\longleftarrow} W \stackrel{f}{\longleftarrow} V$. Again, $\operatorname{Tr}_{q}(g \mathscr{V}$ ect $) \cong \mathbb{F}\left[q^{ \pm 1}\right]$ as $B=\{\mathbb{F}\}$ generates the category additively in a graded sense.

\subsubsection{The Lefschetz number}

Recall that the Lefschetz number $\Lambda\left(f^{\bullet}\right)$ of an endomorphism $f^{\bullet}$ of a chain complex of vector spaces is the alternating sum

$$
\Lambda\left(f^{\bullet}\right):=\sum_{i}(-1)^{i} \operatorname{tr}\left(f^{i}\right)
$$

It is straightforward to check that $\Lambda$ satisfies the trace relation. Even more, Lefschetz numbers of homotopic maps are equal, so that $\Lambda$ descends to a trace on $\operatorname{Com}_{/ h}^{b}(\mathbb{F})$, the homotopy category of complexes. It is the universal triangulated trace as stated in Proposition 2.12. When complexes of graded vector spaces are considered, one constructs the graded Lefschetz number by replacing $\operatorname{tr}$ with $\operatorname{tr}_{q}$ in (2.26).

\subsubsection{The spectrum of a linear map}

The spectrum of an endomorphism $f: V \longrightarrow V$ of a vector space is the set of nonzero eigenvalues. It is an example of a non-linear trace on $\mathscr{V}$ ect. Indeed,

$$
(f \circ g)(x)=\lambda x \quad \Rightarrow \quad(g \circ f)(g(x))=\lambda g(x)
$$

and $g(x) \neq 0$ if $\lambda \neq 0$. Scaling a homomorphism scales elements of its spectrum accordingly, but the spectum of $f+g$ cannot be expressed in general in terms of eigenvalues of $f$ and $g$. 


\subsubsection{Hattori-Stallings trace}

Let $\mathscr{R e p}(A)$ be the category of finitely generated projective right modules over a $\operatorname{ring} A$. The Hattori-Stallings trace $\left[\right.$ Ha65, Sta65] is a homomorphism $t_{P}^{H S}: \operatorname{End}_{A}(P) \longrightarrow A /[A, A]$ defined as the composition

$$
\operatorname{End}_{A}(P) \stackrel{\cong}{\longrightarrow} P \underset{A}{\otimes} P^{*} \stackrel{\epsilon_{P}}{\longrightarrow} A /[A, A]
$$

where $P^{*}=\operatorname{Hom}_{A}(P, A)$ is the left $A$-module dual to $P$ and $A /[A, A]$ is the quotient of $\mathbb{k}$-modules with $[A, A]$ generated by differences $a b-b a$ for all $a, b \in A$. The left isomorphism takes an endomorphism $f$ to $(f \otimes \mathrm{id})(\operatorname{coev}(1))$ and the right map is given as $\epsilon_{P}(x \otimes \alpha):=\alpha(x) .{ }^{3}$ In particular, $\epsilon_{P} \circ(g \otimes$ id $)=\epsilon_{Q} \circ\left(\right.$ id $\left.\otimes g^{*}\right)$ for any $g \in \operatorname{Hom}_{A}(Q, P)$, which guarantees that the trace relation is satisfied. When $P$ is free of finite rank, $t_{P}^{H S}(f)=\sum_{i} f_{i i}$ for $f \in \operatorname{End}_{A}(P)$ represented by the matrix $\left(f_{i j}\right)$.

Projective modules are direct summands of free modules. Hence, $\mathscr{R} e p(A)$ is generated additively by $A$, seen as a right $A$-module, and the universal trace

$$
\operatorname{Tr}(\mathscr{R} e p(A)) \cong \operatorname{Tr}(\mathscr{R} e p A \mid\{A\})=A /[A, A]
$$

coincides with the Hattori-Stallings trace.

\subsubsection{Twisted traces}

Choose an $(A, A)$-bimodule $M$ that is finitely generated and projective as a right module, so that $(-) \otimes_{A} M$ is an endofunctor of $\mathscr{R} e p(A)$. We construct a twisted Hattori-Stallings trace $\operatorname{tr}^{M}$ as the composition

$$
\operatorname{Hom}_{A}(P, P \underset{A}{\otimes} M) \stackrel{c_{P}}{\longrightarrow} P \underset{A}{\otimes} M \underset{A}{\otimes} P^{*} \stackrel{\epsilon_{P}}{\longrightarrow} M /[A, M]=: \operatorname{coInv}(M),
$$

where the $\mathbb{k}$-module $[A, M]$ is generated by differences $a m-m a$ with $a \in A$ and $m \in M$, $c_{P}(f)=(f \otimes \mathrm{id})(\operatorname{coev}(1))$, and $\epsilon_{P}(x \otimes m \otimes \alpha):=\alpha(x) m$. The quotient $\operatorname{coInv}(M)$ is called the space of coinvariants in $M$. Again, the trace condition is satisfied because $\epsilon_{P} \circ(g \otimes \mathrm{id} \otimes \mathrm{id})=\epsilon_{Q} \circ\left(\mathrm{id} \otimes \mathrm{id} \otimes g^{*}\right)$ for any $g \in \operatorname{Hom}_{A}(Q, P)$.

The twisted Hattori-Stallings trace is the universal trace on $(\mathscr{R} e p(A), M)$. Indeed,

$$
\operatorname{Tr}(\mathscr{R} e p(A), M) \cong \operatorname{Tr}(\mathscr{R} e p(A) \mid\{A\}, M) \cong \operatorname{coInv}(M)
$$

by Proposition 2.8, where the right isomorphism arises from the identifications of $\operatorname{End}_{A}(A)$ and $\operatorname{Hom}_{A}(A, M)$ with $A$ and $M$ respectively, each evaluating a function at $1 \in A$.

Given an algebra endomorphism $\varphi \in \operatorname{End}_{\mathrm{Alg}}(A)$ one constructs a twisted trace on $\mathscr{R} e p(A)$ as follows. For a right module $V$ define its $\varphi$-twisting $V_{\bar{\varphi}}:=V \otimes_{A} \varphi$, where ${ }_{\varphi} A$ is obtained from $A$ by redefining the action as $a \cdot x:=\varphi(a) x$. In case $\varphi$ is invertible, there is an isomorphism $V_{\bar{\varphi}} \cong V_{\varphi^{-1}}$ that takes $v \otimes a$ to $v \varphi^{-1}(a)$, where $V_{\varphi^{-1}}$ is obtained from $V$ simply by twisting the action of $A$. If so, a homomorphism $f: W \longrightarrow V_{\bar{\varphi}}$ is precisely a $\mathbb{k}$-linear map $f: W \longrightarrow V$ satisfying $f(w \varphi(a))=f(w) a$. This leads to a twisted Hattori-Stallings trace

$$
\operatorname{tr}^{\varphi}: \mathscr{R e p}(A) \longrightarrow A /[A, A]_{\varphi}
$$

\footnotetext{
${ }^{3}$ Notice the difference between $\epsilon$ and the evaluation map $e v: P^{*} \otimes P \longrightarrow A$, the latter being a homomorphism of $(A, A)$-bimodules.
} 
where $[A, A]_{\varphi}=\operatorname{span}_{\mathbb{k}}\{\varphi(a) b-b a \mid a, b \in A\}$. Notice that $\operatorname{tr}^{\varphi}(f)=\sum_{i} f_{i i}$ for any endomorphism $f$ of a free $A$-module of finite rank. This is the same formula as in the untwisted case, but computed in a different quotient of $A$.

Choose an invertible scalar $q \in \mathbb{k}$. If $A=\bigoplus_{d \in \mathbb{Z}} A_{d}$ is a graded algebra and $M$ a graded bimodule, then the twisted Hattori-Stallings trace can be deformed to take values in the quantum space of coinvariants of $M$

$$
\operatorname{coInv}_{q}(M):=M /[A, M]_{q},
$$

where $[A, M]_{q}=\operatorname{span}_{\mathbb{k}}\left\{a m-q^{d} m a \mid a \in A_{d}, m \in M\right\}$. The details are left to the reader.

\subsubsection{Traces in a pivotal category}

A pivotal category is a monoidal category $\mathscr{C}$ with left duals and a natural isomorphism $\delta_{x}: x \longrightarrow^{* *} x$ for any $x \in \mathscr{C}$. Particular examples are

- the category $\mathscr{V}$ ect of finite dimensional vector spaces together with $\delta=\delta^{\text {vect }}$ the standard isomorphism, and

- the representation category $\mathscr{R} e p(H)$ of a Hopf algebra $H$ with a pivot, a grouplike element $u \in H$ satisfying $S^{2}(h)=u h u^{-1}$; the isomorphism $\delta_{V}$ is given by the formula $\delta_{V}(x)=u \cdot \delta^{\text {vect }}(x)$.

It is common to represent $\mathscr{C}$ diagrammatically: products of objects as sequences of points labeled by the factors, and morphisms as graphs with coupons, read from bottom to top and understood as follows:

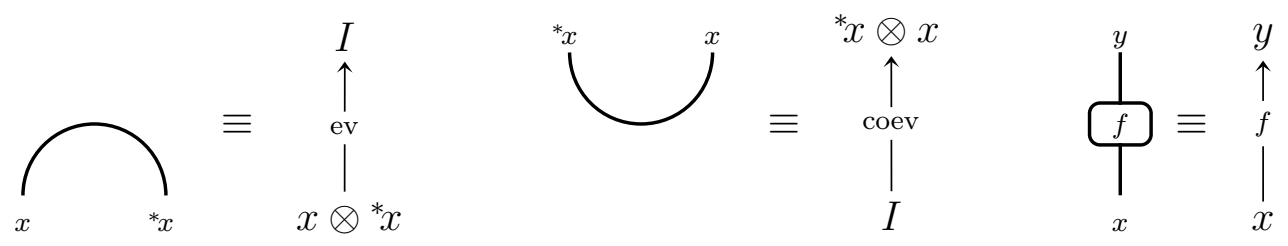

The relations between evaluation and coevaluation translates into straightening zig-zags

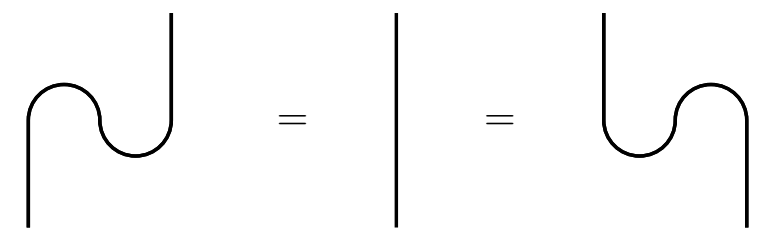

so that isotopic diagrams represent the same morphism. The left and right pivotal traces $t_{\ell}(f)$ and $t_{r}(f)$ of an endomorphism $f \in \mathscr{C}(x, x)$ are defined as the compositions

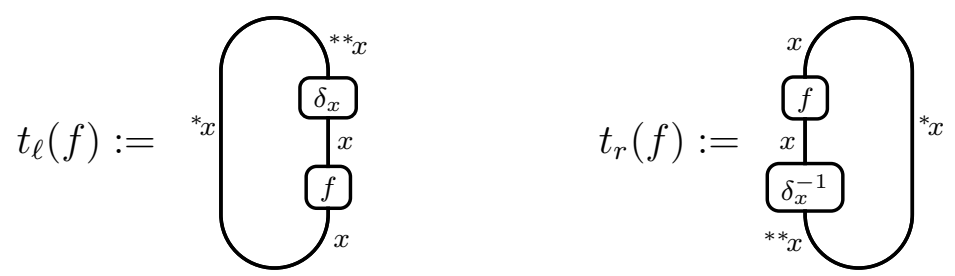

They satisfy left and right version of the trace relation (see the discussion below Definition 2.1)

$$
t_{\ell}\left(f \circ g^{\delta}\right)=t_{\ell}(g \circ f) \quad t_{r}\left(f^{\delta} \circ g\right)=t_{r}(g \circ f)
$$

with $(-)^{\delta}:=\delta \circ * *(-) \circ \delta^{-1}$. The two traces coincide when $\mathscr{C}$ is spherical, but in general they are different. See [BBG18] for more details. 
When $\mathscr{C}=\mathscr{R} e p(H)$ is the representation category of a pivotal Hopf algebra, one can compute the universal right trace

$$
\operatorname{Tr}(\mathscr{R} e p(H), \delta) \cong H / \operatorname{span}_{\mathbb{k}}\left\{S^{2}(y) x-x y \mid x, y \in H\right\}
$$

using the same methods as before. The resemblence with the twisted trace from Section 2.4.6 is not a coincidence: there is a natural isomorphism of functors on $\mathscr{R} e p(H)$

$$
\eta:(-)^{\delta} \longrightarrow(-)_{\bar{u}}, \quad \eta_{V}(x)=x u^{-1},
$$

where $u$ acts on $H$ by conjugation. Indeed, $\eta_{V}(x h)=\left(x u^{-1}\right)\left(u h u^{-1}\right)=\eta_{V}(x) \cdot h$. Analogous statments hold for the left trace.

\subsubsection{The annular closure of a tangle}

Let $q: \mathbb{R}^{2} \times I \longrightarrow \mathbb{R}^{2} \times \mathbb{S}^{1}$ be the quotient map that identifies $(x, y, 0)$ with $(x, y, 1)$ for any $(x, y) \in \mathbb{R}^{2}$. If the tangle $T \subset \mathbb{R}^{2} \times I$ has the same endpoints on both boundary planes, then $q(T)$ is a link in $\mathbb{R}^{2} \times \mathbb{S}^{1}$. It is called the annular closure of $T$, because it can visualized at the level of tangle diagrams by connecting the endpoints inside an annulus, see Figure 2. We consider it as a link $\widehat{T}$ in $\mathbb{R}^{3}$ by using the standard embedding of $\mathbb{R}^{2} \times \mathbb{S}^{1} \cong D^{2} \times \mathbb{S}^{1}$ into the 3 -space.

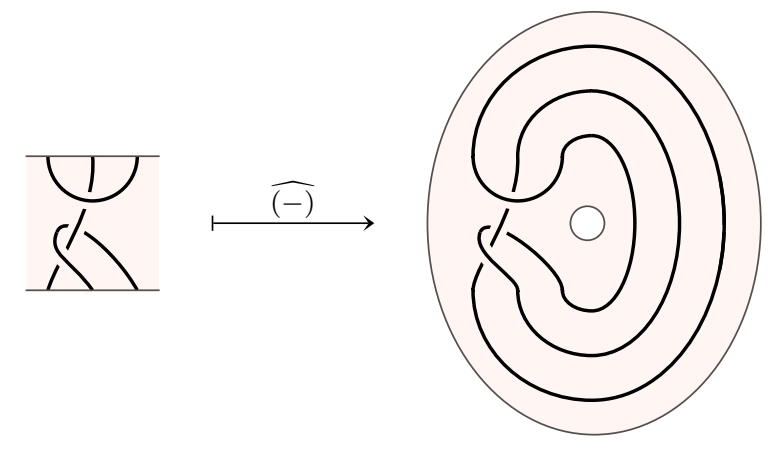

Figure 2: The annular closure of a tangle.

The annular closure described above is a trace function on $\mathscr{T} a n\left(\mathbb{R}^{2}\right)$ valued in $\operatorname{Links}\left(\mathbb{R}^{3}\right)$. Indeed, the links $\widehat{T^{\prime} T}$ and $\widehat{T T^{\prime}}$ are isotopic by rotation.

\subsubsection{Satellite links as traces of tangles}

Embeddings of $\mathbb{R}^{2} \times \mathbb{S}^{1}$ into $\mathbb{R}^{3}$ are parametrized by framed knots - the tubular neighborhood of a knot is homeomorphic to $\mathbb{R}^{2} \times \mathbb{S}^{1}$, but it is the framing that fixes the isotopy class of the homeomorphism. Let $h: \mathbb{R}^{2} \times \mathbb{S}^{1} \longrightarrow \mathbb{R}^{3}$ be the embedding corresponding to a framed knot $K$. The image $h(L)$ of a link $L \subset \mathbb{R}^{2} \times \mathbb{S}^{1}$ is called a satellite link with companion $K$.

Write $K$ as an annular closure of a framed $(1,1)$-tangle $J$. The tangle $J$ describes a tangled embedding of $\mathbb{R}^{2} \times I$ into itself. Let $J_{B}$ be the image under this embedding of the trivial tangle $B \times I$, where $B$ is any set of points on $\mathbb{R}^{2}$. Then $J_{B^{\prime}} T$ and $T J_{B}$ are isotopic for any tangle $T$ with $B$ and $B^{\prime}$ as its bottom and top boundary, where the isotopy slides $T$ along $J$. In other words, $J_{\bullet}$ is a natural transformation of the identity functor on $\mathscr{T} a n\left(\mathbb{R}^{2}\right)$. Using Proposition 2.4 we construct a new trace function $\widehat{(-)}^{J}$ on $\operatorname{Tan}\left(\mathbb{R}^{2}\right)$. It takes a tangle $T$ with same top and bottom boundary to $\widehat{T}^{J}:=h(\widehat{T})$, a satellite knot with compagnion $K$ (see Figure 3 ). 


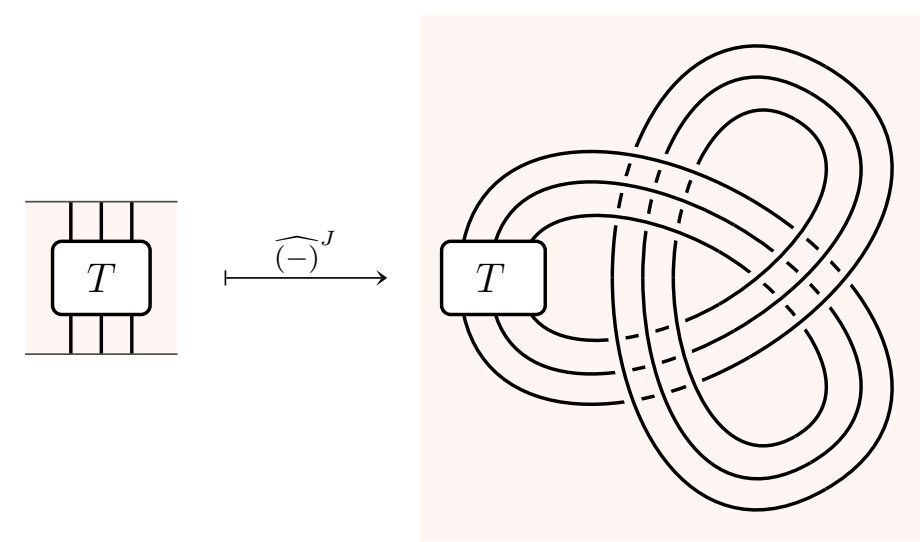

Figure 3: The closure of a $(3,3)$-tangle along a framed trefoil knot.

\subsubsection{Links in surface bundles}

Choose a surface $F$ with a diffeomorphism $\phi \in \operatorname{Diff}(F)$. The mapping torus

$$
M:=F \times I /(p, 1) \sim(\phi(p), 0)
$$

is called the surface bundle with fiber $F$ and monodromy $\phi$. The annular closure of tangles can be generalized to a procedure producing links in $M$.

Given a tangle $T \subset F \times I$ with input $\phi(B)$ and output $B$ we construct its closure $\widehat{T}:=\pi(T)$ in $M$, where $\pi: F \times I \longrightarrow M$ is the quotient map. This operation satisfies the left trace relation with respect to the endofunctor $\phi_{*}$ on $\mathscr{T} a n(F)$ that takes a tangle $T \subset F \times I$ into $(\phi \times$ id $)(T)$. In another words, closing tangles describes a twisted trace $\widehat{(-)}:\left(\operatorname{Tan}(F), \phi_{*}\right) \longrightarrow \operatorname{Links}(M)$.

Theorem 2.19. The closure $\widehat{(-)}$ is the universal left trace on $\left(\operatorname{Tan}(F), \phi_{*}\right)$.

Proof. $\operatorname{Links}(M)$ is a quotient set of $\operatorname{Tr}\left(\operatorname{Tan}(F), \phi_{*}\right)$, because each link in $M$ is a closure of a certain tangle. Suppose that closures $\widehat{T}$ and $\widehat{T}^{\prime}$ are isotopic in $M$. The isotopy can be expressed as a sequence of isotopies supported in small 3-balls, so that we may assume that it fixes some fiber $F^{\prime} \subset M$. Then the cuts $\widehat{T}$ and $\widehat{T}^{\prime}$ along $F^{\prime}$ are isotopic in $F \times I$ and the trace relation implies that the images of $T$ and $T^{\prime}$ in $\operatorname{Tr}^{\ell}\left(\operatorname{Tan}(F), \phi_{*}\right)$ coincide.

\subsubsection{Kauffman Bracket Skein Module}

The Kauffman Bracket Skein Module $\mathcal{S}(M)$ of an oriented $3-$ manifold $M$ is a $\mathbb{Z}\left[A^{ \pm 1}\right]$ module generated by isotopy classes of framed tangles in $M$ (we require the isotopies to fix $\partial M$ if nonempty) modulo the local relations

$$
\begin{aligned}
& \left.\searrow=A \cong+A^{-1}\right)( \\
& \bigcirc=-A^{2}-A^{-2}
\end{aligned}
$$

where the diagrams represent pieces of tangles picked by some small ball, outside of which the tangles coincide. In particular, the circle in the second relation bounds a disk. When $F$ is a surface, then $\mathcal{S}(F \times I)$ is a category and any $\phi \in \operatorname{Diff}(F)$ induces an endofunctor $\phi_{*}$ as in the previous example. The proof of Theorem 2.19 can be easily modified to show that the universal left trace $\operatorname{Tr}^{\ell}\left(\mathcal{S}(F \times I), \phi_{*}\right)$ computes $\delta(M)$, the skein module of the surface bundle $M$ with fiber $F$ and monodromy $\phi$. 


\section{Traces of bicategories}

In this section we categorify twisted traces. We begin with the definition of a preshadow and the construction of the (twisted) horizontal trace of an endobicategory. The universal property and functoriality of the latter are shown in Sections 3.2 and 3.3 respectively, whereas Sections 3.4 and 3.5 discuss two ways to obtain traces from shadows: by restricting to the vertical trace or by decategorifying. These are followed by a material on Lefschetz preshadows and a quantization of this framework. Examples are listed at the end of the section.

\subsection{Categorified traces}

Choose a bicategory $\mathbf{C}$ together with a strong bifunctor $\Sigma: \mathbf{C} \longrightarrow \mathbf{C}$. These come together with natural 2-isomorphisms, the coherence isomorphisms

$$
\begin{array}{ll}
\mathfrak{a}: h \circ(g \circ f) \stackrel{\cong}{\Longrightarrow}(h \circ g) \circ f, & \mathfrak{m}: \Sigma g \circ \Sigma f \stackrel{\cong}{\Longrightarrow} \Sigma(g \circ f), \\
\mathfrak{l}: \operatorname{id}_{y} \circ f \stackrel{\cong}{\Longrightarrow} f, & \mathfrak{i}: \operatorname{id}_{\Sigma x} \stackrel{\cong}{=} \Sigma\left(\operatorname{id}_{x}\right), \\
\mathfrak{r}: f \circ \operatorname{id}_{x} \stackrel{\cong}{\Longrightarrow} f, &
\end{array}
$$

which are often omitted for clarity, see Appendix A.4.

Definition 3.1. A $\Sigma$-twisted preshadow or a preshadow with monodromy $\Sigma$ on $\mathbf{C}$ valued in a category $\mathscr{T}$ is a collection of functors $\langle\langle-\rangle\rangle_{x}: \mathbf{C}(\Sigma x, x) \longrightarrow \mathscr{T}$, one per object $x \in \mathbf{C}$, and natural morphisms $\theta_{g, f}:\langle\langle f \circ \Sigma g\rangle\rangle_{y} \longrightarrow\langle\langle g \circ f\rangle\rangle_{x}$ in $\mathscr{T}$, one for each pair of 1-morphisms $x \stackrel{g}{\longleftarrow} y \stackrel{f}{\longleftarrow} \Sigma x$, such that the following diagrams commute

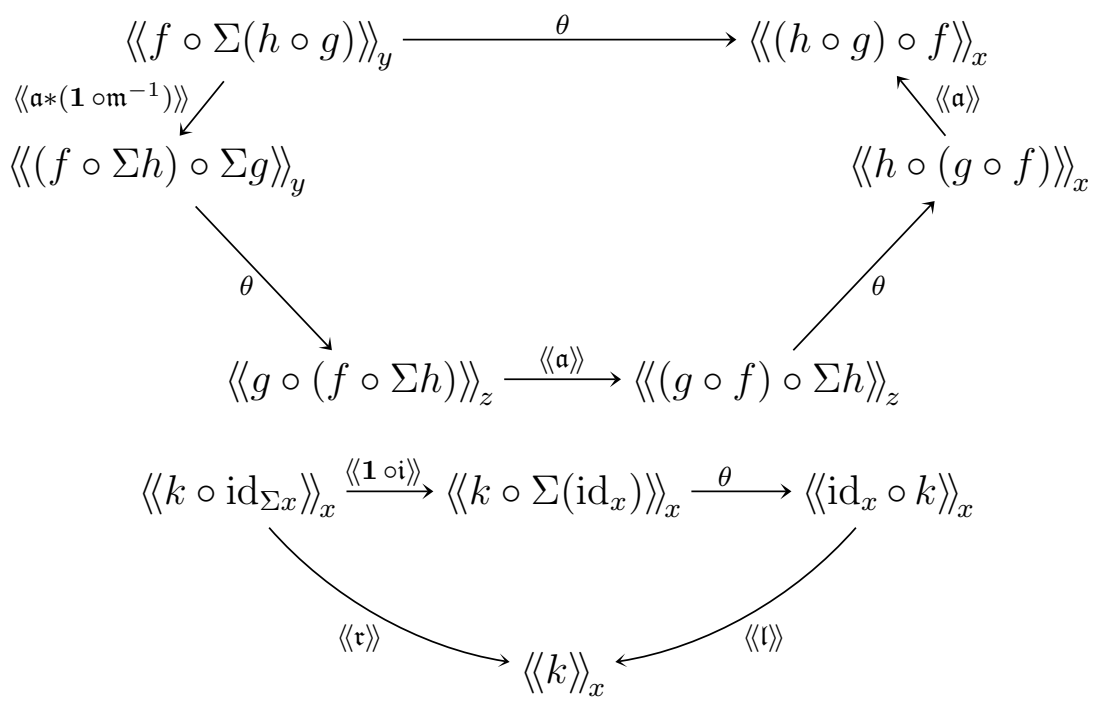

for all $x \stackrel{h}{\longleftarrow} z \stackrel{g}{\longleftarrow} y \stackrel{f}{\longleftarrow} \Sigma x$ and $x \stackrel{k}{\longleftarrow} \Sigma x$. A preshadow is a shadow if each $\theta_{g, f}$ is an isomorphism. It is symmetric when $\theta_{f, g} \circ \theta_{g, f}=\mathrm{id}$.

Remark 3.2. The naturality of $\theta$ means that $\theta_{g^{\prime}, f^{\prime}} \circ\langle\langle\alpha \circ \Sigma \beta\rangle\rangle_{y}=\langle\langle\beta \circ \alpha\rangle\rangle_{x} \circ \theta_{g, f}$ for any 2-morphisms $\alpha: f \Longrightarrow f^{\prime}$ and $\beta: g \Longrightarrow g^{\prime}$.

We often write $\langle\langle-\rangle:(\mathbf{C}, \Sigma) \longrightarrow \mathscr{T}$ for a twisted preshadow on $\mathbf{C}$, despite that it is not a bifunctor. It is understood that when morphism categories $\mathbf{C}(x, y)$ are linear, then so are both $\mathscr{T}$ and the functors $\left\langle\langle-\rangle_{x}\right.$. 
Twisted shadows are categorified versions of traces in the sense, that the equality $t(f \circ \Sigma g)=t(g \circ f)$ defining a (left) twisted trace is replaced by a choice of a natural isomorphism $\langle\langle f \circ \Sigma g\rangle \rrbracket \cong\langle\langle g \circ f\rangle\rangle$. When $\mathbf{C}$ are $\Sigma$ are strict, then the diagrams (3.1) and (3.2) simplify to $\theta_{h \circ g, f}=\theta_{h, g \circ f} \theta_{g, f \circ \Sigma h}$ and $\theta_{\mathrm{id}, k}=\mathrm{id}_{k}$.

The definition of a preshadow coincides with the one of a categorified trace from [HPT15] when $\Sigma=$ Id and $\mathbf{C}$ has only one object, i.e. it is a monoidal category. In comparison to $[\mathrm{PS} 13]$ we do not require $\theta_{f, g} \circ \theta_{g, f}=\mathrm{id}$.

In what follows we construct a twisted preshadow for every bicategory - a generalization of the horizontal trace [BHLZ14]. The next section is devoted to show its functoriality as well as universality.

Definition 3.3. The $\Sigma$-twisted horizontal trace of a bicategory $\mathbf{C}$ is the category $\operatorname{hTr}(\mathbf{C}, \Sigma)$, objects of which are 1-morphisms $f \in \mathbf{C}(\Sigma x, x)$, morphisms from $f \in \mathbf{C}(\Sigma x, x)$ to $g \in \mathbf{C}(\Sigma y, y)$ are equivalence classes $[p, \alpha]$ of squares

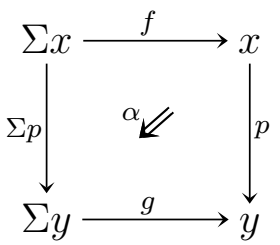

modulo the relation

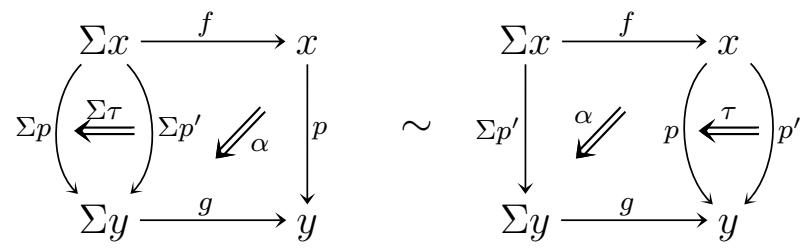

with $\left[\operatorname{id}_{x}, \mathbf{1}_{f}\right]$ the identity on $f$, and the composition

$$
[q, \beta] \circ[p, \alpha]:=\left[q \circ p,\left(\beta \circ \mathbf{1}_{\Sigma p}\right) *\left(\mathbf{1}_{q} \circ \alpha\right)\right]
$$

can be visualized as stacking squares one on top of the other:
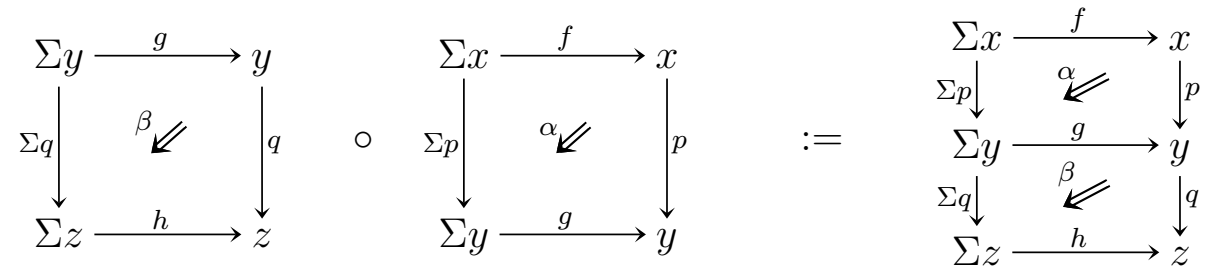

Unitarity and associativity follows from (3.4) with an appropriate composition of associators and unitors as $\tau$.

Proposition 3.4. The horizontal trace is the target of a preshadow $\langle\langle-\rangle\rangle^{\mathrm{h}}$ on $(\mathbf{C}, \Sigma)$ with components $\langle\langle-\rangle\rangle_{x}^{\mathrm{h}}: \mathbf{C}(\Sigma x, x) \longrightarrow \mathrm{h} \operatorname{Tr}(\mathbf{C}, \Sigma)$ the obvious functors and $\theta_{g, f}^{\mathrm{h}}:=[\mathrm{g}, \mathfrak{a}]$. It is a shadow when $\mathbf{C}$ has right duals.

Proof. Commutation of both (3.1) and (3.2) is proven by a direct computation, which is left to the reader. When $\mathbf{C}$ has right duals, consider a morphism represented by

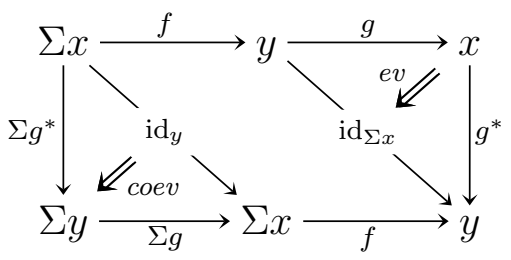


where the triangles are filled with the coevaluation and evaluation 2-morphisms, and the middle parallelogram with a suitable composition of unitors. It is a two-sided inverse of $\theta_{g, f}^{\mathrm{h}}$ due to the relations (A.17) between $e v$ and coev.

The idea underlying the definition of the horizontal trace is to represent 1-endomorphisms by boundary circles of a cylinder, the interior of which carries a 2-morphism. Indeed, such a cylinder can be obtained by gluing the vertical boundaries of the square (3.3) together, but keeping the seam line, see Figure 4. The seam is cooriented and

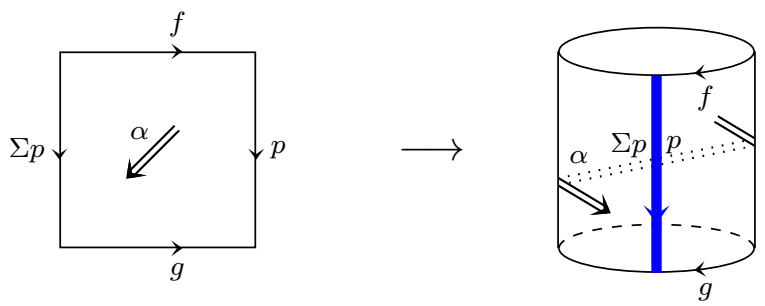

Figure 4: Visualization of the horizontal trace.

a 2-morphism can "slide" through it, in which case it is acted upon by $\Sigma$ as described by (3.4). The morphism $\theta^{\mathrm{h}}$ is represented in this picture by the cylinder obtained from the identity on $g \circ f$ by applying a half-twist to its top boundary circle, see Figure 5.

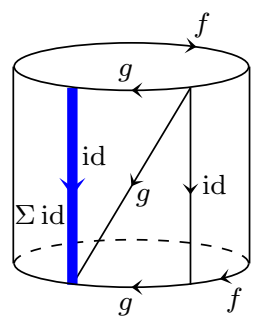

$\underset{\text { by } \pi \text { clockwise }}{\stackrel{\text { twist the top circle }}{\longrightarrow}}$

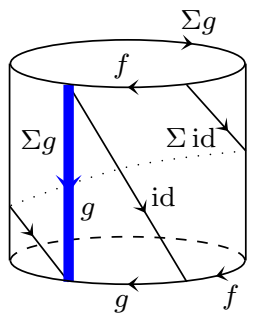

Figure 5: Visualization of the cyclicity morphism in hTr.

The symmetric horizontal trace (i.e. for $\Sigma=\mathrm{Id}$ ) is also called the annularization functor [MW10]. Its generalization to any surface is known as factorization homology [BZBJ15].

\subsection{The universal property of the horizontal trace}

Let $\langle\langle-\rangle\rangle:(\mathbf{C}, \Sigma) \longrightarrow \mathscr{T}$ and $\langle\langle-\rangle\rangle^{\prime}:(\mathbf{C}, \Sigma) \longrightarrow \mathscr{T}^{\prime}$ be two preshadows. We say that $\langle\langle-\rangle\rangle^{\prime}$ factorizes through $\langle\langle-\rangle\rangle$ if there exist a functor $T: \mathscr{T} \longrightarrow \mathscr{T}^{\prime}$ and a collection of natural isomorphisms $\eta_{x}: T \circ\langle\langle-\rangle\rangle_{x} \longrightarrow\langle\langle-\rangle\rangle_{x}^{\prime}$ such that $\eta_{x} \circ T\left(\theta_{g, f}\right)=\theta_{g, f}^{\prime} \circ \eta_{y}$ for any $x \stackrel{g}{\longleftarrow} y \stackrel{f}{\longleftarrow} \Sigma x$. Factorizations $(T, \eta)$ and $\left(T^{\prime}, \eta^{\prime}\right)$ are equivalent if there exists a natural isomorphism $\epsilon: T \longrightarrow T^{\prime}$ such that $\eta_{x}=\eta_{x}^{\prime} *\left(\epsilon \circ \mathbf{1}_{\langle-\rangle\rangle}\right)$, i.e.

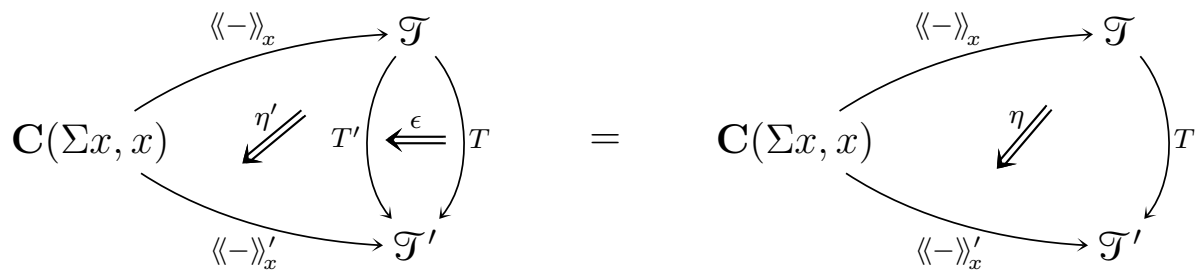

The following result states that the horizontal trace twisted by $\Sigma$ is the universal $\Sigma$ twisted preshadow. 
Theorem 3.5. If $\mathbf{C}$ has left duals, then every preshadow on $(\mathbf{C}, \Sigma)$ factorizes through $\mathrm{h} \operatorname{Tr}(\mathbf{C}, \Sigma)$ uniquely up to an equivalence.

Proof. Given a preshadow $\langle\langle-\rangle:(\mathbf{C}, \Sigma) \longrightarrow \mathscr{T}$ we construct a functor $T: h \operatorname{Tr}(\mathbf{C}, \Sigma) \longrightarrow \mathscr{T}$ by taking $f: \Sigma x \longrightarrow x$ to $\langle\langle f\rangle\rangle_{x}$ and a morphism $[p, \alpha]: f \longrightarrow g$ to the composition

$$
\begin{aligned}
& \langle\langle f\rangle\rangle_{x} \stackrel{\langle\text { coev } \circ \mathbf{1}\rangle_{x}}{\longrightarrow}\left\langle\left\langle{ }^{*} p \circ p \circ f\right\rangle\right\rangle_{x} \stackrel{\langle\mathbf{1} \circ \alpha\rangle\rangle_{y}}{\longrightarrow}\left\langle\left\langle{ }^{*} p \circ g \circ \Sigma p\right\rangle\right\rangle_{x} \\
& \stackrel{\theta_{p,{ }^{*} \circ \circ g}}{\longrightarrow}\left\langle\left\langle p \circ{ }^{*} p \circ g\right\rangle\right\rangle_{y} \stackrel{\langle\langle e v \circ \mathbf{1}\rangle\rangle_{y}}{\longrightarrow}\langle\langle g\rangle\rangle_{y},
\end{aligned}
$$

where associators and unitors are omitted for clarity. Notice that $\langle\langle-\rangle\rangle=T \circ\langle\langle-\rangle\rangle^{\mathrm{h}}$ and $\theta_{g, f}=T\left(\theta_{g, f}^{\mathrm{h}}\right)$. For uniqueness, suppose that $\left(T^{\prime}, \eta\right):\langle\langle-\rangle\rangle^{\mathrm{h}} \longrightarrow\langle\langle-\rangle\rangle$ is another factorization of a morphism of shadows with each $\eta_{x}$ an isomorphism. Then $\eta$ is a natural isomorphism of functors $\eta: T^{\prime} \longrightarrow T$, because $\langle\langle f\rangle\rangle^{\mathrm{h}}=f$ and $T f=\langle\langle f\rangle\rangle$; naturality follows from (3.9) and the observation that a similar sequence determines $T^{\prime}([p, \alpha])$.

Corollary 3.6. Suppose $\mathbf{C}$ has both left and right duals. Then any preshadow on $\mathbf{C}$ is a shadow.

Proof. It follows from Theorem 3.5 that a preshadow $(\langle\langle-\rangle\rangle, \theta)$ factorizes through the horizontal trace. In particular, $\theta$ is an image of $\theta^{\mathrm{h}}$, which is an isomorphism by Proposition 3.4.

\subsection{Functoriality}

To discuss functoriality of hTr we begin with constructing a suitable 3-category of endobicategories.

A morphism of endobicategories $(\mathbf{F}, \omega):(\mathbf{C}, \Sigma) \longrightarrow\left(\mathbf{C}^{\prime}, \Sigma^{\prime}\right)$ consists of a strong bifunctor $\mathbf{F}: \mathbf{C} \longrightarrow \mathbf{C}^{\prime}$, so that the coherence 2-morphisms $\mathfrak{m}_{\mathbf{F}}: \mathbf{F} g \circ \mathbf{F} f \Longrightarrow \mathbf{F}(g \circ f)$ and $\mathfrak{i}_{\mathbf{F}}: \operatorname{id}_{\mathbf{F} x} \Longrightarrow \mathbf{F}\left(\mathrm{id}_{x}\right)$ are invertible, and a weak natural transformation $\omega: \Sigma^{\prime} \mathbf{F} \longrightarrow \mathbf{F} \Sigma$. The latter comes with a collection of 2-morphisms

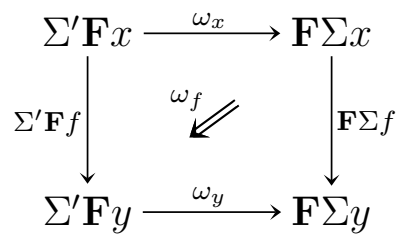

parametrized with 1-morphisms $f \in \mathbf{C}(x, y)$. The 2-morphisms $\omega_{f}$ are not required to be invertible. If they are, we say that $(\mathbf{F}, \omega)$ is strong.

Suppose that $(\mathbf{F}, \omega)$ and $\left(\mathbf{F}^{\prime}, \omega^{\prime}\right)$ are morphisms between the same endobicategories. A natural transformation $(\eta, \mathfrak{n}):(\mathbf{F}, \omega) \longrightarrow\left(\mathbf{F}^{\prime}, \omega^{\prime}\right)$ consists of a strong natural transformation of bifunctors $\eta: \mathbf{F} \longrightarrow \mathbf{F}^{\prime}$ and a natural 2-morphism $\mathfrak{n}_{x}: \eta_{\Sigma x} \circ \omega_{x} \Longrightarrow \omega_{x}^{\prime} \circ \Sigma^{\prime} \eta_{x}$ that - in addition to the usual coherence conditions - fits into the commutative hexagon

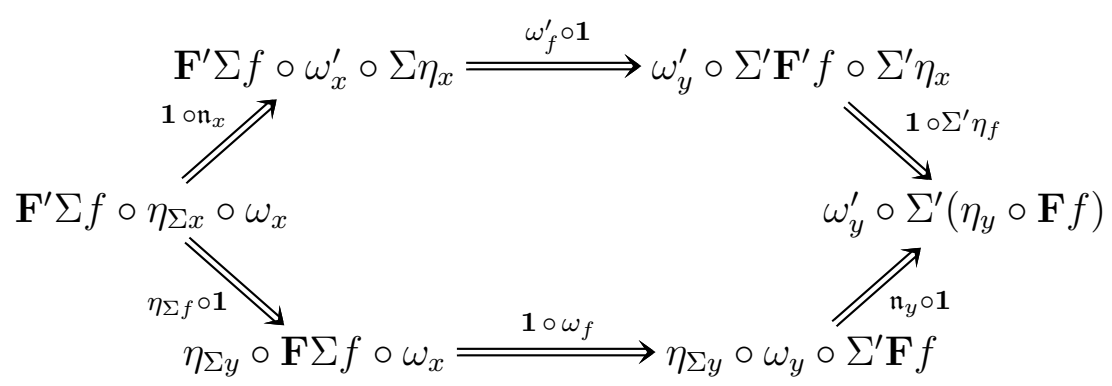


for any 1-morphism $f \in \mathbf{C}(x, y)$, where canonical isomorphisms are omitted.

Finally, we require that a modification $\Gamma: \eta \longrightarrow \eta^{\prime}$ of natural transformations $(\eta, \mathfrak{n})$ and $\left(\eta^{\prime}, \mathfrak{n}^{\prime}\right)$ satisfies $\left(\mathbf{1} \circ \Sigma \Gamma_{x}\right) * \mathfrak{n}_{x}=\mathfrak{n}_{x}^{\prime} *\left(\Gamma_{\Sigma^{\prime} x} \circ \mathbf{1}\right)$ in addition to the usual coherence condition.

Definition 3.7. We write EndoBicat for the 3-category of endobicategories, morphisms, natural transformations and modifications, as described above. Its restriction to endobicategories with left duals is denoted by EndoBicat*.

Preshadows can be pulled back along morphisms of endobicategories. Indeed, given a preshadow $\langle\langle-\rangle\rangle$ on $\left(\mathbf{C}^{\prime}, \Sigma^{\prime}\right)$ and a morphism $(\mathbf{F}, \omega):(\mathbf{C}, \Sigma) \longrightarrow\left(\mathbf{C}^{\prime}, \Sigma^{\prime}\right)$ we define a its pullback $\left(\mathbf{F}^{*}\langle\langle-\rangle\rangle, \mathbf{F}^{*} \theta\right)$ on $(\mathbf{C}, \Sigma)$ as follows:

$$
\begin{aligned}
& \mathbf{F}^{*}\langle\langle f\rangle\rangle_{x}:=\left\langle\left\langle\mathbf{F} f \circ \omega_{x}\right\rangle\right\rangle_{F x} \\
& \mathbf{F}^{*}\langle\langle\alpha\rangle\rangle_{x}:=\langle\langle\mathbf{F} \alpha \circ \mathbf{1}\rangle\rangle_{F x}
\end{aligned}
$$

with the cyclicity morphism $\left(\mathbf{F}^{*} \theta\right)_{g, f}$ the composition

$$
\begin{aligned}
& \mathbf{F}^{*}\langle\langle f \circ \Sigma g\rangle\rangle_{y}=\left\langle\left\langle\mathbf{F}(f \circ \Sigma g) \circ \omega_{y}\right\rangle\right\rangle_{F y} \stackrel{\left.\left\langle\mathfrak{m}_{\mathbf{F}}^{-1} \circ \mathbf{1}\right\rangle\right\rangle}{\longrightarrow}\left\langle\left\langle\mathbf{F} f \circ \mathbf{F} \Sigma g \circ \omega_{y}\right\rangle\right\rangle_{F y} \\
& \stackrel{\left.\left\langle\mathbf{1} \circ \omega_{g}\right\rangle\right\rangle}{\longrightarrow}\left\langle\left\langle\mathbf{F} f \circ \omega_{x} \circ \Sigma^{\prime} \mathbf{F} g\right\rangle\right\rangle_{F y}
\end{aligned}
$$

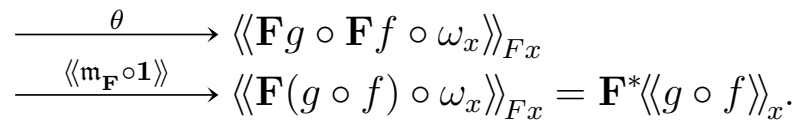

Notice that the second arrow is invertible when $(\mathbf{F}, \omega)$ is a strong morphism.

Proposition 3.8. The datum $\left(\mathbf{F}^{*}\langle\langle-\rangle\rangle, \mathbf{F}^{*} \theta\right)$ is a preshadow on $(\mathbf{C}, \Sigma)$. It is a shadow when $(\mathbf{F}, \omega)$ is a strong morphism and $(\langle\langle-\rangle, \theta)$ is a shadow.

Proof. Left as an exercise.

Let $\langle\langle-\rangle\rangle,\langle\langle-\rangle\rangle^{\prime}:(\mathbf{C}, \Sigma) \longrightarrow \mathscr{T}$ be two preshadows. A collection of natural transformations $\eta_{x}:\langle\langle-\rangle\rangle_{x} \longrightarrow\langle\langle-\rangle\rangle_{x}^{\prime}$ is a morphism of preshadows if it is coherent with the cyclicity morphisms: $\theta_{g, f}^{\prime} \circ \eta_{y}=\eta_{x} \circ \theta_{g, f}$. There is a morphism of pullback shadows associated to any natural transformation of morphisms of endobicategories

$(\mathbf{C}, \Sigma)$

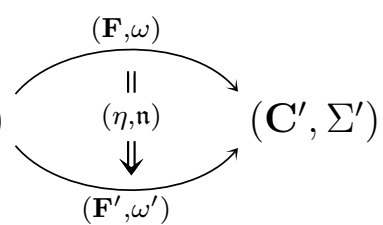

Indeed, there is a morphism in $h \operatorname{Tr}\left(\mathbf{C}^{\prime}, \Sigma^{\prime}\right)$

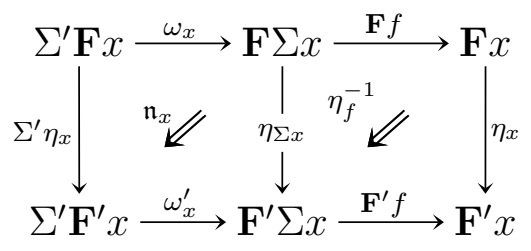

which, assuming that $\mathbf{C}^{\prime}$ has left duals, descend to a morphism $(\eta, \mathfrak{n})_{f}$ in $\mathscr{T}$ between $\mathbf{F}^{*}\langle\langle f\rangle\rangle$ and $\mathbf{F}^{*}\langle\langle f\rangle\rangle$ for any shadow $\langle\langle-\rangle\rangle$ on $\left(\mathbf{C}^{\prime}, \Sigma^{\prime}\right)$.

Lemma 3.9. The collection $(\eta, \mathfrak{n})_{*}$ is a morphism of shadows from $\mathbf{F}^{*}\langle\langle-\rangle\rangle$ to $\mathbf{F}^{*}\langle\langle-\rangle\rangle$. Morphisms induced by isomorphic transformations are equal. 
Proof. The naturality and compatibility with cyclicity morphisms can be checked in $\mathrm{h} \operatorname{Tr}\left(\mathbf{C}^{\prime}, \Sigma^{\prime}\right)$. They follow directly from the coherence conditions of $\eta_{f}$ and $\mathfrak{n}_{x}$. The details are left as an exercise.

Everything together implies functoriality of the horizontal trace. Let EndoBicat ${ }^{*, \leqslant 2}$ be the restriction of EndoBicat* to a 2-category, obtained by forgetting modifications and identifying isomorphic natural transformations.

Theorem 3.10 (Functoriality of the horizontal trace). The horizontal trace hTr extends to a strict 2-functor $\mathrm{hTr}$ : EndoBicat ${ }^{*, \leqslant 2} \longrightarrow$ Cat, which assigns to a morphism of endobicategories $(\mathbf{F}, \omega):(\mathbf{C}, \Sigma) \longrightarrow\left(\mathbf{C}^{\prime}, \Sigma^{\prime}\right)$ the functor $\mathrm{h} \operatorname{Tr}(\mathbf{F}, \omega): \mathrm{h} \operatorname{Tr}(\mathbf{C}, \Sigma) \longrightarrow \mathrm{h} \operatorname{Tr}\left(\mathbf{C}^{\prime}, \Sigma^{\prime}\right)$ that takes an object $f: \Sigma x \longrightarrow x$ into $\mathbf{F} f \circ \omega_{x}$ and a morphism $[p, \alpha]$ to the class of the diagram

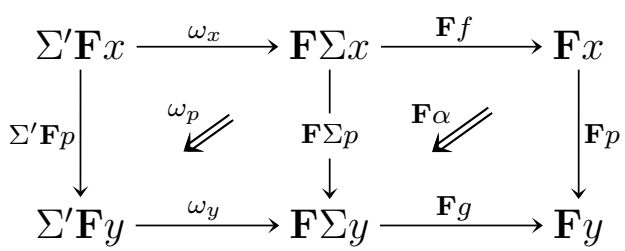

and a natural transformation $(\eta, \mathfrak{n})$ is sent to the natural transformation $\mathrm{h} \operatorname{Tr}(\eta, \mathfrak{n})$ with components (3.13).

Proof. Apply Theorem 3.5 to the pullback along $(\mathbf{F}, \omega)$ of the universal preshadow on $\left(\mathbf{C}^{\prime}, \Sigma^{\prime}\right)$ to construct $\operatorname{hTr}(\mathbf{F}, \omega)$. The coherence condition for a natural transformation guarantees that $h \operatorname{Tr}(\eta, \mathfrak{n})$ is a morphism of shadows.

Corollary 3.11. Let $(\mathbf{F}, \omega):(\mathbf{C}, \Sigma) \longrightarrow\left(\mathbf{C}^{\prime}, \Sigma^{\prime}\right)$ be a local equivalence, i.e. $\omega$ is invertible and $\mathbf{F}$ restricts to equivalences of morphism categories $\mathbf{C}(x, y) \approx \mathbf{C}^{\prime}(\mathbf{F} x, \mathbf{F} y)$ for all objects $x, y \in \mathbf{C}$. Then $\mathrm{h} \operatorname{Tr}(\mathbf{F}, \omega)$ is full and faithful.

Proof. By replacing $\mathbf{C}^{\prime}$ with the image of $\mathbf{F}$ we may assume that the bifunctor is a biequivalence. Then $\mathrm{h} \operatorname{Tr}(\mathbf{F}, \omega)$ is an equivalence of categories by Theorem 3.10 and Lemma 3.9, hence, full and faithful.

\subsection{A connection with the vertical trace}

Assume now that $\Sigma$ fixes objects, i.e. $\Sigma x=x$ for any object $x \in$ C. Then $\Sigma$ can be restricted to each morphism category $\mathbf{C}(x, y)$. Replacing each of them by its trace $\operatorname{Tr}(\mathbf{C}(x, y), \Sigma)$ results in the vertical trace $\operatorname{vTr}(\mathbf{C}, \Sigma)$ : a category with the same objects as $\mathbf{C}$, morphisms twisted trace classes of 2-morphisms $\alpha: f \Longrightarrow \Sigma f$, and composition induced by the horizontal composition of 2-morphisms. The composition in $\operatorname{vTr}(\mathbf{C}, \Sigma)$ is both unital and associative, because the trace class of a morphism is invariant under conjugation.

There is a natural functor $v \operatorname{Tr}(\mathbf{C}, \Sigma) \longrightarrow h \operatorname{Tr}(\mathbf{C}, \Sigma)$, defined by expanding objects to identity morphisms. Explicitly,

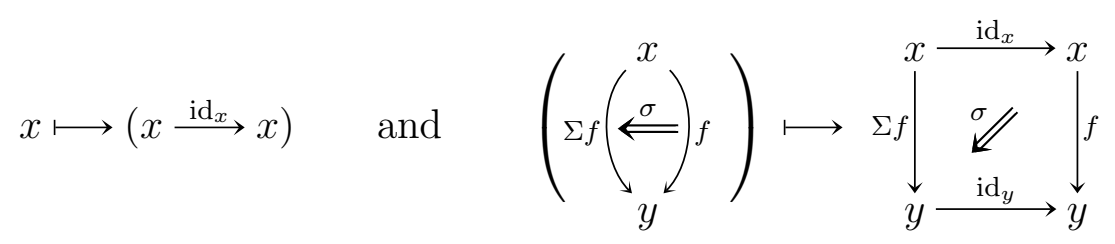

It is clearly full and faithful, but not necessarily surjective on objects. 
When $\mathbf{C}$ has left duals, then the above functor can be used to restrict a preshadow $\langle\langle-\rangle\rangle:(\mathbf{C}, \Sigma) \longrightarrow \mathscr{T}$ to a trace $t^{\langle-\rangle}:(\mathbf{C}(x, y), \Sigma) \longrightarrow \mathscr{T}\left(\left\langle\left\langle\mathrm{id}_{x}\right\rangle\right\rangle,\left\langle\left\langle\mathrm{id}_{y}\right\rangle\right\rangle\right)$ using the formula

$$
t^{《-\rangle}(p \stackrel{\alpha}{\longrightarrow} \Sigma p):=\langle\langle[p, \alpha]\rangle\rangle
$$

where we identify $\langle\langle-\rangle\rangle$ with the corresponding functor on $\operatorname{hTr}(\mathbf{C}, \Sigma)$. The following is an immediate consequence of this construction.

Proposition 3.12. The restriction to $\mathbf{C}(x, y)$ of the universal $\Sigma$-twisted preshadow is the universal $\Sigma$-twisted trace.

\subsection{Decategorification}

An $n$-category $\mathbf{C}$ can be truncated to an $(n-1)$-category by forgetting its highest level morphisms and identifying isomorphic $(n-1)$-morphisms. Thence, we have a functor $\Pi: n$ Cat $\longrightarrow(n-1)$ Cat. Clearly, this construction applies to endo- $n$-categories as well. We are mostly interested in the case $n=1,2$.

Applying $\Pi$ to a shadow $\langle\langle-\rangle\rangle: \mathbf{C} \longrightarrow \mathscr{T}$ results in a (left) trace $\Pi\langle\langle-\rangle\rangle: \Pi \mathbf{C} \longrightarrow \Pi \mathscr{T}$. Conversely, any left trace on $\Pi \mathbf{C}$ can be lifted to a shadow if $\mathbf{C}$ is small. ${ }^{4}$

Lemma 3.13. Choose a small endobicategory $(\mathbf{C}, \Sigma)$ and a (left) trace $\operatorname{tr}:(\Pi \mathbf{C}, \Pi \Sigma) \longrightarrow S$ for some set $S$. There exists a shadow $T:(\mathbf{C}, \Sigma) \longrightarrow \mathcal{S}$, such that $\Pi \mathcal{S}=S$ and $\Pi T=\operatorname{tr}$.

Proof. In what follows we write $\operatorname{dom}(\alpha):=f$ and $\operatorname{cod}(\alpha):=f^{\prime}$ for the domain and codomain of a 2-morphism $\alpha: f \Longrightarrow f^{\prime}$. Let $\tilde{\mathcal{S}}$ be the category with $\operatorname{Ob}(\tilde{\mathcal{S}})=S$ and morphisms $\tilde{S}(s, t)$ the finite sequences $\left(\alpha_{n}, \ldots, \alpha_{1}\right)$ of 2 -morphisms $\alpha_{i} \in \mathbf{C}\left(\Sigma x_{i}, x_{i}\right)$ satisfying $\operatorname{tr}\left(\operatorname{dom}\left(\alpha_{i+1}\right)\right)=\operatorname{tr}\left(\operatorname{cod}\left(\alpha_{i}\right)\right)$ for $i=1, \ldots, n-1$, such that $s=\operatorname{tr}\left(\operatorname{dom}\left(\alpha_{1}\right)\right)$ and $t=\operatorname{tr}\left(\operatorname{cod}\left(\alpha_{n}\right)\right)$. Composition of morphisms is defined as concatenation of sequences. The category $\delta$ is a quotient of $\tilde{S}$ by the relations

$$
\begin{aligned}
(\ldots, \beta, \alpha, \ldots) & \sim(\ldots, \beta * \alpha, \ldots) \quad \text { whenever } \beta * \alpha \text { exists, and } \\
(\ldots, \mathbf{1}, \ldots) & \sim(\ldots, \ldots) .
\end{aligned}
$$

They allow us to reduce a given sequence of 2-morphism to a sequence of noncomposable morphisms, none of which is the identity. An easy application of the Bergman Diamond Lemma [Be78] shows that this reduced sequence is unique. In particular,

$$
\left(\beta_{m}, \ldots, \beta_{1}\right) \circ\left(\alpha_{1}, \ldots, \alpha_{n}\right) \sim()
$$

implies $m=n$ and each $\beta_{i} \circ \alpha_{i}=1$ if both sequences are reduced. Hence, each $\alpha_{i}$ is a 2-isomorphism in $\mathbf{C}$ if $\left(\alpha_{1}, \ldots, \alpha_{n}\right)$ is an isomorphism in $\delta$, which implies that $\operatorname{tr}\left(\operatorname{dom}\left(\alpha_{i}\right)\right)=\operatorname{tr}\left(\operatorname{cod}\left(\alpha_{i}\right)\right)$ and

$$
\operatorname{dom}\left(\alpha_{n}, \ldots, \alpha_{1}\right)=\operatorname{tr}\left(\operatorname{dom}\left(\alpha_{1}\right)\right)=\operatorname{tr}\left(\operatorname{cod}\left(\alpha_{n}\right)\right)=\operatorname{cod}\left(\alpha_{n}, \ldots, \alpha_{1}\right) .
$$

Therefore, $\delta$ does not have isomorphisms between different objects, and $\Pi \delta=S$. The desired shadow $T:(\mathbf{C}, \Sigma) \longrightarrow \mathcal{S}$ is defined as $T(f):=\operatorname{tr}(f)$ and $T(\alpha):=(\alpha)$.

The above result allows us to formally prove that the horizontal trace is a categorification of the universal trace of a category. Despite proving it only for small bicategories, we believe that with a slight modification of our argument the result can be generalized to locally small bicategories.

\footnotetext{
${ }^{4}$ A bicategory is small if its 2-morphisms (and so objects as well as 1-morphisms) form a set.
} 
Theorem 3.14. There is a natural bijection $\Pi(\mathrm{h} \operatorname{Tr}(\mathbf{C}, \Sigma)) \approx \operatorname{Tr}^{\ell}(\Pi \mathbf{C}, \Pi \Sigma)$ for each small endobicategory $(\mathbf{C}, \Sigma)$ with left and right duals.

Proof. Because $\mathbf{C}$ has right duals, $\operatorname{hTr}(\mathbf{C}, \Sigma)$ is a shadow and we can apply $\Pi$ to get a trace $\Pi(h \operatorname{Tr}(\mathbf{C}, \Sigma))$. We must show that it is the universal left trace, i.e. that every left trace $t:(\Pi \mathbf{C}, \Pi \Sigma) \longrightarrow S$ factorizes through it. For that use Lemma 3.13 to find a shadow $T:(\mathbf{C}, \Sigma) \longrightarrow \delta$ that lifts $t$. According to Theorem 3.5, $T$ factorizes through $h \operatorname{Tr}(\mathbf{C}, \Sigma)$, and applying $\Pi$ results in a desired factorization of $t$.

Thence, the horizontal trace is a categorification of the universal left trace. Right traces are categorified by a dual version of shadows, defined on morphism categories $\mathbf{C}(x, \Sigma x)$. The convention for the twisting of $\mathrm{hTr}$ used in this paper is justified by the relation of hTr with the vertical trace discussed in Section 3.4.

\subsection{Categorified Lefschetz traces}

Let $\mathrm{Com}_{/ h}^{b}(\mathbf{C})$ be a bicategory obtained from a locally additive bicategory $\mathbf{C}$ by replacing each morphism category $\mathbf{C}(x, y)$ with the corresponding homotopy category of bounded complexes $\mathrm{Com}_{/ h}^{b}(\mathbf{C}(x, y))$. It is locally triangulated, i.e. the functors $f \circ(-)$ and $(-) \circ f$ preserve distinguished triangles and commute with homological degree shifts. Having chosen an endobifunctor $\Sigma$ on $\mathbf{C}$ we extend it naturally over $\mathrm{Com}_{/ h}^{b}(\mathbf{C})$.

Definition 3.15. A preshadow $\langle\langle-\rangle\rangle:\left(\operatorname{Com}_{/ h}^{b}(\mathbf{C}), \Sigma\right) \longrightarrow \mathscr{T}$ is triangulated if $\mathscr{T}$ is a triangulated category and the components $\langle\langle-\rangle\rangle_{x}$ are triangulated functors.

A twisted preshadow $\langle\langle-\rangle\rangle:(\mathbf{C}, \Sigma) \longrightarrow \mathscr{T}$ can be extended to a twisted triangulated preshadow $\langle\langle-\mid\rangle\rangle: \operatorname{Com}_{/ h}^{b}(\mathbf{C})(\Sigma x, x) \longrightarrow \operatorname{Com}_{/ h}^{b}(\mathscr{T})$, which we call the Lefschetz preshadow induced by $\langle\langle-\rangle\rangle$. For each object $x \in \mathbf{C}$ the functor $\langle\langle-\mid\rangle\rangle_{x}$ is constructed by applying $\langle\langle-\rangle\rangle_{x}$ component-wise:

$$
\left.\left\langle\left\langle f^{\bullet}\right\rangle\right\rangle\right\rangle_{x}=\left(\ldots \longrightarrow\left\langle\left\langle f^{i}\right\rangle\right\rangle_{x} \stackrel{\left\langle\langle d\rangle_{x}\right.}{\longrightarrow}\left\langle\left\langle f^{i+1}\right\rangle\right\rangle_{x} \stackrel{\langle\langle d\rangle\rangle_{x}}{\longrightarrow}\left\langle\left\langle f^{i+2}\right\rangle\right\rangle_{x} \longrightarrow \ldots\right)
$$

and likewise for 2-morphisms. Notice that $\left\langle\left\langle\left\langle g^{\bullet} \circ f^{\bullet}\right\rangle\right\rangle_{x}^{i}=\bigoplus_{p+q=i}\left\langle\left\langle g^{p} \circ f^{q}\right\rangle\right\rangle_{x}\right.$ for a pair of 1-morphisms $x \stackrel{g^{\bullet}}{\longleftarrow} y \stackrel{f^{\bullet}}{\longleftarrow} \Sigma x$. We check below that the sums

$$
\Theta_{g^{\bullet}, f}^{i}:=\sum_{p+q=i}(-1)^{p q} \theta_{g^{p}, f^{q}}
$$

are components of a formal chain map $\Theta_{g^{\bullet}, f} \bullet:\left\langle\left\langle f^{\bullet} \circ \Sigma g^{\bullet}\right\rangle\right\rangle_{x} \longrightarrow\left\langle\left\langle\left\langle g^{\bullet} \circ f^{\bullet}\right\rangle\right\rangle\right\rangle_{y}$. It is invertible if $(\langle\langle-\rangle\rangle, \theta)$ is a shadow.

Lemma 3.16. The datum $(《\langle-\rangle\rangle, \Theta)$ is a triangulated preshadow on $\operatorname{Com}_{/ h}^{b}(\mathbf{C})$. It is a shadow if so is $\langle\langle-\rangle$.

Proof. The functors $\langle\langle-\rangle\rangle_{x}$ are additive and, as such, they preserve mapping cones. Hence, they are triangulated. To check that $\Theta_{g^{\bullet}, f} \bullet$ is a chain map, we compute

$$
\begin{aligned}
d_{g \bullet \circ f}^{i} \bullet \circ \Theta_{g^{\bullet}, f}^{i} \bullet & =\sum_{p+q=i}(-1)^{p q}\left\langle\left\langle d_{g^{\bullet}}^{p} \circ \mathrm{id}+(-1)^{p} \mathrm{id} \circ d_{f}^{q} \bullet\right\rangle \circ \theta_{g^{p}, f^{q}}\right. \\
& =\sum_{p+q=i}(-1)^{p q}\left(\theta_{g^{p+1}, f^{q}} \circ\left\langle\left\langle\mathrm{id} \circ d_{\Sigma g^{\bullet}}^{p}\right\rangle\right\rangle+(-1)^{p} \theta_{g^{p}, f^{q+1}} \circ\left\langle\left\langle d_{f}^{q} \bullet \mathrm{id}\right\rangle\right\rangle\right)
\end{aligned}
$$




$$
=\sum_{p+q=i+1}(-1)^{p q} \theta_{g^{p}, f^{q}} \circ\left\langle\left\langle(-1)^{q} \mathrm{id} \circ d_{\Sigma g^{\bullet}}^{p}+d_{f}^{q} \bullet \circ \mathrm{id}\right\rangle\right\rangle=\Theta_{g^{\bullet}, f}^{i+1} \bullet \circ d_{f}^{i} \bullet \Sigma g^{\bullet}
$$

and the naturality follows from the naturality of $\theta$ :

$$
\begin{aligned}
\Theta_{g_{1}, f_{1}^{\bullet}}^{i} \circ\left\langle\left\langle\left\langle\alpha^{\bullet} \circ \Sigma \beta^{\bullet}\right\rangle\right\rangle\right\rangle^{i} & =\sum_{p+q=i}(-1)^{p q} \theta_{g_{1}^{p}, f_{1}^{q}} \circ\left\langle\left\langle\alpha^{p} \circ \Sigma \beta^{q}\right\rangle\right\rangle \\
& \left.=\sum_{p+q=i}(-1)^{p q}\left\langle\left\langle\beta^{q} \circ \alpha^{p}\right\rangle\right\rangle \circ \theta_{g_{0}^{p}, f_{0}^{q}}=\left\langle\left\langle\beta^{\bullet} \circ \alpha^{\bullet}\right\rangle\right\rangle\right\rangle^{i} \circ \Theta_{g_{0}^{\bullet}, f_{0}^{\bullet}}^{\bullet}
\end{aligned}
$$

for any 2-morphisms $\alpha^{\bullet}: f_{0}^{\bullet} \longrightarrow f_{1}^{\bullet}$ and $\beta^{\bullet}: g_{0}^{\bullet} \longrightarrow g_{1}^{\bullet}$. Finally, commutativity of (3.1) and (3.2) can be easily checked component-wise.

Recall that a triangulated trace is additive with respect to (twisted) endomorphisms of distinguished triangles. An analoguous statement holds for Lefschetz preshadows.

Proposition 3.17. Let $\mathbf{C}$ have left duals and choose a commuting diagram in $\mathrm{Com}_{/ h}^{b}(\mathbf{C})$

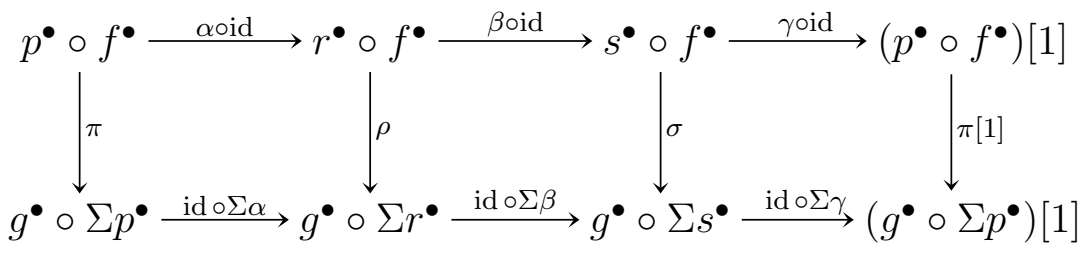

where $f^{\bullet} \in \operatorname{Com}_{/ h}^{b}(\mathbf{C})(\Sigma x, x), g^{\bullet} \in \operatorname{Com}_{/ h}^{b}(\mathbf{C})(\Sigma y, y)$, and $p^{\bullet} \stackrel{\alpha}{\longrightarrow} r^{\bullet} \stackrel{\beta}{\longrightarrow} s^{\bullet} \stackrel{\gamma}{\longrightarrow} p^{\bullet}[1]$ is a distinguished triangle in $\operatorname{Com}_{/ h}^{b}(\mathbf{C})(x, y)$. Then $\langle\langle[r, \rho]\rangle\rangle=\langle\langle[p, \pi]\rangle\rangle+\langle\langle\langle[s, \sigma]\rangle\rangle$.

Proof. The 1-morphism $s^{\bullet}$ is homotopy equivalent to the mapping cone of $\alpha: p^{\bullet} \longrightarrow r^{\bullet}$. In particular, $\sigma^{n}:\left(s^{\bullet} \circ f^{\bullet}\right)^{n} \longrightarrow\left(g^{\bullet} \circ \Sigma s^{\bullet}\right)^{n}$ consists of components

$$
\begin{array}{ll}
\sigma_{p, p}: p^{j+1} \circ f^{i} \longrightarrow g^{i^{\prime}} \circ \Sigma p^{j^{\prime}+1} & \sigma_{r, p}: r^{j} \circ f^{i} \longrightarrow g^{i^{\prime}} \circ \Sigma p^{j^{\prime}+1} \\
\sigma_{p, r}: p^{j+1} \circ f^{i} \longrightarrow g^{i^{\prime}} \circ \Sigma r^{j^{\prime}} & \sigma_{r, r}: r^{j} \circ f^{i} \longrightarrow g^{i^{\prime}} \circ \Sigma r^{j^{\prime}}
\end{array}
$$

for $i+j=i^{\prime}+j^{\prime}=n$. A simple diagram chasing shows that $\sigma_{p, p}$ and $\sigma_{r, r}$ are chain maps homotopic to $\pi[1]$ and $\rho$ respectively. We can thus take $\sigma_{p, p}^{\bullet}=\pi[1]$ and $\sigma_{r, r}^{\bullet}=\rho^{\bullet}$.

Recall that the left dual to $\left(C^{\bullet}, d\right)$ is given by the complex

$$
\ldots \longrightarrow{ }^{*} C^{-i-1} \stackrel{*^{-i}}{\longrightarrow}{ }^{*} C^{-i} \stackrel{{ }^{*} d^{-i+1}}{\longrightarrow}{ }^{*} C^{-i+1} \longrightarrow \ldots
$$

with ${ }^{*} C^{-i}$ in homological degree $i$. The evaluation and coevaluation maps do not vanish only in homological degree zero, where they are given by the obvious maps

$$
A \stackrel{\sum \operatorname{coev}_{C^{i}}}{\longrightarrow} \bigoplus_{i}^{*} C^{i} \otimes C^{i}=\left({ }^{*} C^{\bullet} \otimes C^{\bullet}\right)^{0}
$$

and

$$
\left(C^{\bullet} \otimes{ }^{*} C^{\bullet}\right)^{0}=\bigoplus_{i} C^{i} \otimes{ }^{*} C^{i} \stackrel{\sum e{ }^{i}}{\longrightarrow} A
$$

In particular, $s^{j}=p^{j+1} \oplus r^{j}$ leads to the decomposition

$$
\left({ }^{*} s\right)^{-j} \circ s^{j}=\left({ }^{*} p^{j+1} \circ p^{i+j}\right) \oplus\left({ }^{*} r^{j} \circ r^{j}\right) \oplus\left({ }^{*} p^{j+1} \circ r^{j}\right) \oplus\left({ }^{*} r^{j} \circ p^{j+1}\right) .
$$


The coevaluation takes values only in the first two terms, which are also the only components on which the evaluation does not vanish. Hence, the only components of $\langle\langle[s, \sigma]\rangle \rrbracket$ that contribute are

$$
\left\langle\left\langle f^{i}\right\rangle\right\rangle \stackrel{\langle\text { coevo } \mathbf{1}\rangle\rangle}{\longrightarrow}\left\langle\left\langle p^{*} p^{j+1} \circ p^{j+1} \circ f^{i}\right\rangle\right\rangle \stackrel{\left.\left\langle\mathbf{1} \circ \sigma_{p, p}\right\rangle\right)}{\stackrel{(-1)^{(i+j) j} \theta}{\longrightarrow}}\left\langle\left\langle{ }^{*} p^{j+1} \circ g^{i} \Sigma \circ p^{j+1} \circ{ }^{*} p^{j+1} \circ g^{i}\right\rangle\right\rangle \stackrel{\langle e v \circ \mathbf{1}\rangle)}{\longrightarrow}\left\langle\left\langle g^{i}\right\rangle\right\rangle,
$$

and those with $p^{j+1}$ replaced by $r^{j}$. Because $\sigma_{p, p}^{\bullet}=\pi[1] \bullet$ and $\sigma_{r, r}^{\bullet}=\rho^{\bullet}$, the compositions (3.28) (resp. those with $r^{j}$ instead of $p^{j+1}$ ) coincide with the components of $\left.\langle\langle[p[1], \pi[1]]\rangle\rangle\right\rangle$ (resp. $\langle\langle[r, \rho]\rangle\rangle)$. Hence, $\langle\langle[s, \sigma]\rangle\rangle=\langle\langle\langle[r, \rho]\rangle\rangle+\langle\langle[p[1], \pi[1]]\rangle\rangle\rangle=\langle\langle\langle[r, \rho]\rangle\rangle\rangle-\langle\langle\langle[p, \pi]\rangle\rangle$.

Corollary 3.18. Assume that $\mathbf{C}$ has left duals and $\Sigma$ fixes objects of $\mathbf{C}$. Then the Lefschetz preshadow $\langle\langle-\rangle\rangle$ on $\mathrm{Com}_{/ h}^{b}(\mathbf{C})$ induced by $\langle\langle-\rangle\rangle$ restricts to the Lefschetz trace on $\mathrm{Com}_{/ h}^{b}(\mathbf{C}(x, y))$ induced by $t^{《-\rangle}$, the trace defined as in (3.15).

Proof. The restricted trace is triangulated due to Proposition 3.17 applied to the case $f=\mathrm{id}_{x}$ and $g=\mathrm{id}_{y}$. Hence, the thesis follows from Proposition 2.12.

\subsection{Deformation of categorified traces}

A bicategory $\mathbf{C}$ is locally pregraded if each morphism category $\mathbf{C}(x, y)$ is pregraded, the horizontal composition preserves the degree of 2-morphisms, and the canonical 2isomorphisms $\mathfrak{a}, \mathfrak{l}$, and $\mathfrak{r}$ are homogeneous of degree 0 . As usual, we write $|\alpha|$ for the degree of a homogeneous 2-morphism $\alpha$. We say that $\mathbf{C}$ is locally graded if each morphism category $\mathbf{C}(x, y)$ comes with a translation functor $\{1\}$ that is an equivalence, such that for any pair of composable 1-morphism $x \stackrel{f}{\longrightarrow} y \stackrel{g}{\longrightarrow} z$ the horizontal compositions $g \circ f\{1\}$, $g\{1\} \circ f$, and $(g \circ f)\{1\}$ are naturally isomorphic. Likewise for categories, both frameworks are equivalent: a locally pregraded bicategory can be formally extended to a locally graded one, and vice versa. In what follows we choose the pregraded framework.

Let $(\mathbf{C}, \Sigma)$ be a locally pregraded endobicategory. This means that $\mathbf{C}$ is locally pregraded and $\Sigma$ is graded, i.e. it preserves the degree of 2-morphisms and the structural 2-isomorphisms $\mathfrak{m}$ and $\mathfrak{i}$ are homogeneous in degree 0 .

Definition 3.19. A $\Sigma$-twisted quantum preshadow on $\mathbf{C}$ valued in a pregraded $\mathbb{k}\left[q^{ \pm 1}\right]-$ linear category $\mathscr{T}$ is a collection of graded functors $\langle\langle-\rangle\rangle_{x}: \mathbf{C}(\Sigma x, x) \longrightarrow \mathscr{T}$ together with graded morphisms $\theta_{g, f}:\langle\langle f \circ \Sigma g\rangle\rangle_{y} \longrightarrow\langle\langle g \circ f\rangle\rangle_{x}$ in $\mathscr{T}$, one for each pair of 1 -morphisms $x \stackrel{g}{\longleftarrow} y \stackrel{f}{\longleftarrow} \Sigma x$, which are natural in the graded sense, i.e.

$$
\theta_{g^{\prime}, f^{\prime}} \circ\langle\langle\alpha \circ \Sigma \beta\rangle\rangle=q^{|\beta|}\langle\langle\beta \circ \alpha\rangle\rangle \circ \theta_{g, f}
$$

for homogeneous 2-morphisms $\alpha: f \Longrightarrow f^{\prime}$ and $\beta: g \Longrightarrow g^{\prime}$, and such that the defining diagrams for preshadows (3.1) and (3.2) commute. We say that $(\langle\langle-\rangle, \theta)$ is a quantum shadow if each $\theta_{f, g}$ is invertible.

Likewise in the case of traces, quantum preshadows arise by extending coefficients of $\mathbf{C}$ to $\mathbb{k}\left[q^{ \pm 1}\right]$ and deforming $\Sigma$ into $\Sigma_{q}$ by redefining it on homogeneous 2 -morphisms as

$$
\Sigma_{q}(\alpha):=q^{-|\alpha|} \Sigma(\alpha)
$$

Quantum preshadows on $(\mathbf{C}, \Sigma)$ are then precisely preshadows on $\left(\mathbf{C}, \Sigma_{q}\right)$. It follows that there is a deformed twisted horizontal trace $\operatorname{hTr}_{q}(\mathbf{C}, \Sigma)$, the quantum $\Sigma$-twisted horizontal 
trace of $\mathbf{C}$, obtained by deforming the defining relation into

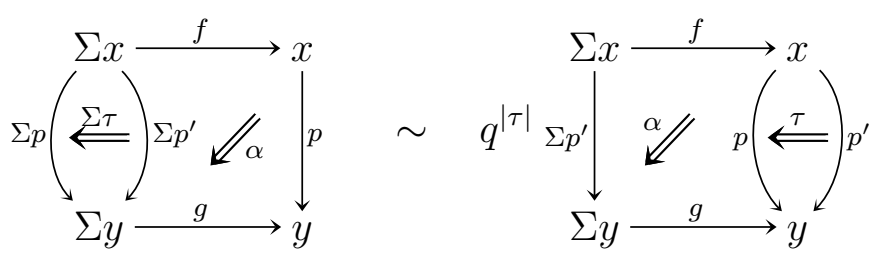

where $\alpha$ and $\tau$ are homogeneous. When $\mathbf{C}$ is locally graded, then, in an analogy to traces of categories, the $\mathbb{k}\left[q^{ \pm 1}\right]$-linear structure on $\operatorname{hTr}_{q}(\mathscr{C}, \Sigma)$ satisfies $q \cdot[p, \alpha]:=[p\{1\}, \alpha\{1\}]$. Diagrammatically,

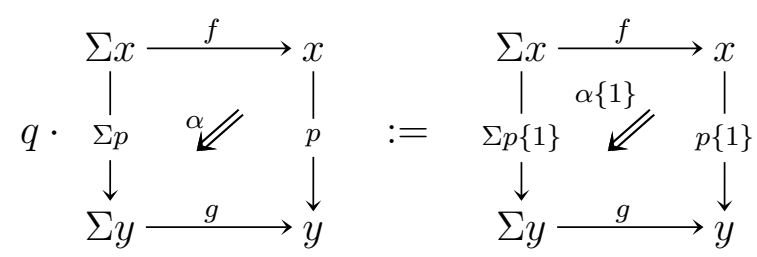

The following is a direct generalization from the undeformed framework. In particular, every $\Sigma$-twisted quantum preshadow factorizes through $\operatorname{hT}_{q}(\mathbf{C}, \Sigma)$ uniquely up to an equivalence.

Proposition 3.20. Assume that $\mathbf{C}$ has left duals. Then $\mathrm{hTr}_{q}(\mathbf{C}, \Sigma)$ with $\langle\langle-\rangle\rangle_{x}^{\mathrm{h}}$ the obvious functors is the universal $\Sigma$-twisted quantum preshadow. It is a quantum shadow if $\mathbf{C}$ has right duals.

Locally pregraded endobicategories form a 3-category if we require all bifunctor to preserve degrees of 2-morphisms and all structural 2-morphisms (such as $\omega_{f}$ in the definition of a morphism or $\eta_{f}$ and $\mathfrak{n}_{x}$ in the definition of a natural transformation) to be graded. A pullback of a twisted quantum preshadow along a morphism of locally pregraded endobicategories is again a twisted quantum preshadow, and natural transformations of such morphisms induce morphisms of preshadows. The following is an immediate consequence of that and the universality of $\mathrm{hTr}_{q}$.

Theorem 3.21 (Functoriality of the quantum horizontal trace). The quantum horizontal trace $\mathrm{hTr}_{q}$ extends to a strict 2-functor from the 2-category of locally pregraded endobicategories with duals to pregraded categories.

We omit the explicit construction of the 2-functor, because it is a verbatim copy of the one from Theorem 3.10.

\subsection{Examples}

\subsubsection{The twisted trace}

Choose a pair of functors $\mathscr{C} \longleftarrow F \mathscr{D} G \mathscr{C}$. The pair $(G$, id) is a morphism of endocategories, from $(\mathscr{C}, F G)$ to $(\mathscr{D}, G F)$, and as such it induces a map between their universal traces

$$
\operatorname{Tr}(G, \mathrm{id}): \operatorname{Tr}(\mathscr{C}, F G) \longrightarrow \operatorname{Tr}(\mathscr{D}, G F), \quad \operatorname{tr}(f) \longmapsto \operatorname{tr}(G f) .
$$

To show naturality, choose natural transformations $\eta: F \longrightarrow F^{\prime}$ and $\mu: G \longrightarrow G^{\prime}$. They determine morphisms of endocategories

$$
(\mathrm{Id}, \mu \circ F \eta):(\mathscr{C}, F G) \longrightarrow\left(\mathscr{C}, F^{\prime} G^{\prime}\right)
$$




$$
(\mathrm{Id}, \eta \circ G \mu):(\mathscr{D}, G F) \longrightarrow\left(\mathscr{D}, G^{\prime} F^{\prime}\right)
$$

and hence maps on traces. Using $\mu \circ F \eta=F^{\prime} \eta \circ \mu$ we check directly that

$$
\begin{aligned}
\left(\operatorname{Tr}\left(G^{\prime}, \mathrm{Id}\right) \circ \operatorname{Tr}(\mathrm{Id}, \mu \circ F \eta)\right)(\operatorname{tr}(f))=\operatorname{tr}\left(G^{\prime} F^{\prime} \eta \circ G^{\prime} \mu \circ G^{\prime} f\right)= \\
\operatorname{tr}\left(G^{\prime}(\mu \circ f) \circ \eta\right)=\operatorname{tr}(\eta \circ G(\mu \circ f))=(\operatorname{Tr}(\mathrm{Id}, \eta \circ G \mu) \circ \operatorname{Tr}(G, \mathrm{id}))(\operatorname{tr}(f)) .
\end{aligned}
$$

Because $\operatorname{tr}(F G f)=\operatorname{tr}(f)$ by Lemma 2.2, the above upgrades $\operatorname{Tr}(-)$ to a symmetric shadow on Cat, the 2-category of small categories. The same argument shows that $\operatorname{Tr}_{q}(-)$ is a quantum shadow on gCat, the 2-category of small graded categories, with the cyclicity map satisfying $\operatorname{tr}(F G f)=\operatorname{tr}(F G f \circ \mathrm{id})=q^{|f|} \operatorname{tr}(f)$ for a homogeneous $f$.

Although not every functor has a left dual (left dualizable functors are exactly those with a left adjoint), $\operatorname{Tr}(-)$ factors through $\mathrm{h} \operatorname{Tr}\left(\right.$ Cat) (resp. $\operatorname{Tr}_{q}(-)$ factors through $\left.\mathrm{hTr}_{q}(\mathbf{g C a t})\right)$. Indeed, a representant $(F, \omega)$ of a morphism in the horizontal trace is precisely a morphism of endocategories, and it can be checked directly that $\operatorname{Tr}(F, \omega)$ (resp. $\left.\operatorname{Tr}_{q}(F, \omega)\right)$ depends only on the image of $(F, \omega)$ in the horizontal trace; see also Theorem 4.5 .

\subsubsection{The space of coinvariants}

Write Bimod for the bicategory of $\mathbb{k}$-algebras, bimodules, and bimodule maps; the horizontal composition of bimodules $N \in \operatorname{Bimod}(A, B)$ and $N^{\prime} \in \operatorname{Bimod}(B, C)$ is defined as their tensor product: $N^{\prime} \circ N:=N \otimes_{B} N^{\prime}$. It admits a symmetric shadow that assigns to an $(A, A)$-bimodule $M$ the space of coinvariants $\operatorname{coInv}(M):=M /[A, M]$ and the cyclicity morphism of which is the standard twist: $\theta_{N^{\prime}, N}\left(\left[n^{\prime} \otimes n\right]\right)=\left[n \otimes n^{\prime}\right]$, where $N \in \operatorname{Bimod}(A, B)$ and $N^{\prime} \in \operatorname{Bimod}(B, A)$.

Likewise, the quantum space of coinvariants $\operatorname{coInv}_{q}(-)$ defined in (2.32) determines a shadow on gBimod, the locally graded bicategory of graded algebras, graded bimodules, and graded bimodule maps. The cyclicity morphism is a noninvolutive deformation of the standard twist: $\theta_{N^{\prime}, N}\left(\left[n^{\prime} \otimes n\right]\right)=q^{\left|n^{\prime}\right|}\left[n \otimes n^{\prime}\right]$ for homogeneous $n^{\prime} \in N^{\prime}$ and $n \in N$.

Let $\mathbf{g R e p}$ be the restriction of $\mathbf{g B i m o d}$ to those bimodules that are finitely generated and projective as right modules. It can be realized as a sub-2-category of gCat by interpreting an algebra $A$ as its representation category gRep $(A)$, and an $(A, B)$-bimodule $N$ as the functor $(-) \otimes_{A} N$. Therefore, it admits a preshadow as described in the previous example, which factorizes through $\mathrm{hTr}_{q}$ (gRep). Explicitly, it assigns to

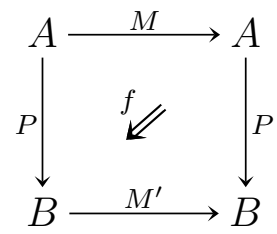

the linear map $[m] \longmapsto \operatorname{tr}_{q}^{P}(p \longmapsto f(m \otimes p))$, where $\operatorname{tr}_{q}^{P}$ is the quantum deformation of the twisted Hattori-Stallings trace from Section 2.4.5. We claim that this shadow coincides with the one restricted from Bimod. Indeed, given $N \in \operatorname{gR} \operatorname{ep}(A, B)$ and $N^{\prime} \in \operatorname{gRep}(B, A)$ with homogeneous $n \in N$ and $n^{\prime} \in N^{\prime}$, the cyclicity map takes the class of $\left[n^{\prime} \otimes n\right]$ to

$$
\begin{aligned}
& \operatorname{tr}_{q}^{N^{\prime}}\left(n^{\prime} \otimes n \otimes \operatorname{id}_{N^{\prime}}\right)=\epsilon_{q, N^{\prime}}\left(n^{\prime} \otimes n \otimes \operatorname{coev}_{N^{\prime}}(1)\right)= \\
& \quad=q^{\left|n^{\prime}\right|}\left[n \otimes\left(\operatorname{id}_{N^{\prime}} \otimes e v_{N^{\prime}}\right)\left(\operatorname{coev}_{N^{\prime}}(1) \otimes n^{\prime}\right)\right]=q^{\left|n^{\prime}\right|}\left[n \otimes n^{\prime}\right]=\theta_{N^{\prime}, N}\left(\left[n^{\prime} \otimes n\right]\right) .
\end{aligned}
$$




\subsubsection{Twisted coinvariants}

Let $\widetilde{\mathbf{B i m o d}}$ be an extension of Bimod, objects of which are pairs $(A, \varphi)$ consisting of an algebra $A$ and its automorphism $\varphi$, and whose 1- and 2-morphisms are bimodules and bimodule maps respectively with no restriction imposed. It can be upgraded to an endobicategory via an endobifunctor $\Sigma$ that affects only 1-morphisms:

$$
\Sigma N:={ }_{\bar{\varphi}} N_{\bar{\psi}} \cong A_{\varphi} \underset{A}{\otimes} N \underset{B}{\otimes}{ }_{\psi} B
$$

for $N \in \widetilde{\operatorname{Bimod}}(A, \varphi ; B, \psi)$, where $A_{\varphi}$ is the $(A, A)$-bimodule $A$ with the right action $x \cdot a:=x \varphi(a)$, and likewise for ${ }_{\psi} B$. For any object $(A, \varphi)$ there is an isomorphism of bimodules $A \cong{ }_{\varphi} A_{\varphi}$ that takes $a$ to $\varphi(a)$. Hence, $\Sigma\left(N \otimes_{B} N^{\prime}\right) \cong \Sigma N \otimes_{A} \Sigma N^{\prime}$ as desired.

Twisted spaces of coinvariants from Section 2.4.6 constitute a shadow on Bimod with the cyclicity map

$$
\begin{aligned}
& \operatorname{coInv}^{\psi}\left(\Sigma N^{\prime}{\underset{A}{\otimes}} N\right)=\operatorname{coInv}\left({ }_{\psi} B_{\psi} \underset{B}{\otimes} N^{\prime}{\underset{A}{\otimes}}_{\varphi} A \underset{A}{\otimes} N\right) \cong \\
& \operatorname{coInv}\left({ }_{\varphi} A \underset{A}{\otimes} N \underset{B}{\otimes} N^{\prime}\right)=\operatorname{coInv}^{\varphi}\left(N \underset{B}{\otimes} N^{\prime}\right)
\end{aligned}
$$

for $N \in \widetilde{\boldsymbol{B}} \boldsymbol{\operatorname { i m o d }}(A, \varphi ; B, \psi)$ and $N^{\prime} \in \widetilde{\boldsymbol{B} i m o d}(B, \psi ; A, \varphi)$. Recalling that $\Sigma N^{\prime}$ and $N^{\prime}$ coincide as $\mathbb{k}$-modules, we find out that (3.34) is the twist $\left[n^{\prime} \otimes n\right] \longmapsto\left[n \otimes n^{\prime}\right]$.

Let $\widetilde{\mathbf{R} e p} \subset \widetilde{\mathbf{B i m o d}}$ be the restriction to bimodules that are finitely generated and projective as right modules. Such an $(A, B)$-bimodule $N$ has a left dual ${ }^{*} N:=\operatorname{Hom}_{B}(N, B)$, the $(B, A)$-bimodule of right $B$-linear functions on $N .^{5}$ Thus the above shadow can be restricted to a trace on $\mathrm{v} \operatorname{Tr}(\widetilde{\boldsymbol{R} e p}(A, \varphi ; B, \psi), \Sigma)$ that takes $f \in \operatorname{Hom}(N, \Sigma N)$ to the composition

$$
\begin{aligned}
\operatorname{coInv}^{\varphi}(A) \stackrel{\text { coev }}{\longrightarrow} \operatorname{coInv}^{\varphi}\left(N \underset{B}{\otimes}{ }^{*} N\right) \stackrel{f \otimes i \mathrm{id}}{\longrightarrow} \operatorname{coInv}^{\psi}\left(\Sigma N \underset{B}{\otimes}{ }^{*} N\right) \\
\stackrel{\theta}{\longrightarrow} \operatorname{coInv}^{\psi}\left({ }^{*} N \underset{A}{\otimes} N\right) \stackrel{e v}{\longrightarrow} \operatorname{coInv}^{\psi}(B),
\end{aligned}
$$

which, when evaluated at $[a] \in \operatorname{coInv}^{\varphi}(A)$, recovers the $\psi$-twisted Hattori-Stallings trace of $f_{a}(n):=f(a n)$.

\subsubsection{Hochschild homology}

Let $M$ be an $(A, A)$-bimodule and write $R_{\bullet}(A)$ for the bar resolution of $A$ [Lo, 1.1.11]. The Hochschild homology of $A$ with coefficients in $M$ or, shortly, the Hochschild homology of $M$ is the homology of the chain complex $C H_{\bullet}(A, M):=\operatorname{coInv}\left(M \otimes_{A} R_{\bullet}(A)\right)$, to which we refer as the Hochschild complex. Explicitly, $C_{n}(A, M)=M \otimes A^{\otimes n}$ and the Hochschild differential is the alternating sum

$$
\begin{aligned}
\partial\left(m \otimes a_{1} \otimes \cdots \otimes\right. & \left.a_{n}\right)=m a_{1} \otimes a_{2} \otimes \cdots \otimes a_{n} \\
+ & \sum_{i=1}^{n-1}(-1)^{i} m \otimes a_{1} \otimes \cdots \otimes a_{i} a_{i+1} \otimes \cdots \otimes a_{n} \\
& +(-1)^{n} a_{n} m \otimes a_{1} \otimes \cdots \otimes a_{n-1} .
\end{aligned}
$$

${ }^{5}$ Notice that ${ }^{*} N$ is projective as a left module, but not necessarily as a right one. Hence, in general, it is not a 1-morphism in $\widetilde{\mathbf{R e p}}$. 
In particular, $H H_{0}(A, M) \cong \operatorname{coInv}(M)$ is the ordinary space of coinvariants.

Hochschild homology is not directly a shadow on Bimod: for that one has to extend it to derived categories of bimodules and use the derived tensor product as the composition. It is not necessary in the case of Rep, because an $(A, A)$-bimodule of the form $N \otimes_{B} N^{\prime}$ has two projective resolutions

$$
P_{\bullet}\left(N, N^{\prime}\right):=R_{\bullet}(A) \underset{A}{\otimes}\left(N \underset{B}{\otimes} N^{\prime}\right)
$$

and

$$
R_{\bullet}\left(N, N^{\prime}\right):=\operatorname{Tot}\left(R_{\bullet}(A) \underset{A}{\otimes} N \underset{B}{\otimes} R_{\bullet}(B) \underset{B}{\otimes} N^{\prime}\right)
$$

which, by the uniqueness of the resolution, are homotopy equivalent. Therefore, there is a sequence of homotopy equivalences

$$
C H_{\bullet}\left(B, N^{\prime} \underset{A}{\otimes} N\right) \simeq \operatorname{coInv}\left(R_{\bullet}\left(N^{\prime}, N\right)\right) \cong \operatorname{coInv}\left(R \bullet\left(N, N^{\prime}\right)\right) \simeq C H_{\bullet}\left(A, N \underset{B}{\otimes} N^{\prime}\right),
$$

where the middle map is the standard twist $\left[\underline{b} \otimes n^{\prime} \otimes \underline{a} \otimes n\right] \longmapsto\left[\underline{a} \otimes n \otimes \underline{b} \otimes n^{\prime}\right]$, in which $\underline{a}$ stands for a tensor product of elements of $A$, and likewise for $\underline{b}$. Doing this twice results in a map chain homotopic to the identity, so that $\mathrm{CH}_{\bullet}$ is a symmetric shadow on Rep.

Let $\varphi \in \operatorname{Aut}(A)$. The $\varphi$-twisted Hochschild complex $C H_{\bullet}^{\varphi}(A, M)$ of an $(A, A)$-bimodule $M$ is obtained by replacing $M$ with ${ }_{\varphi} M$ (or, equivalently, coInv with $\operatorname{coInv}^{\varphi}$ ):

$$
C H_{\bullet}^{\varphi}(A, M):=\operatorname{coInv}\left({ }_{\varphi} M \underset{A}{\otimes} R_{\bullet}(A)\right) .
$$

It coincides with $C H_{\bullet}(A, M)$ except that the last term in the differential is replaced with

$$
(-1)^{n} \varphi\left(a_{n}\right) m \otimes a_{1} \otimes \cdots \otimes a_{n-1} .
$$

Hochschild chains can be visualized by oriented circles divided into segments, one labeled with $m \in M$ and the others with $a_{0}, \ldots, a_{n}$. Each of the terms of the differential merges two segments multiplying their labels:

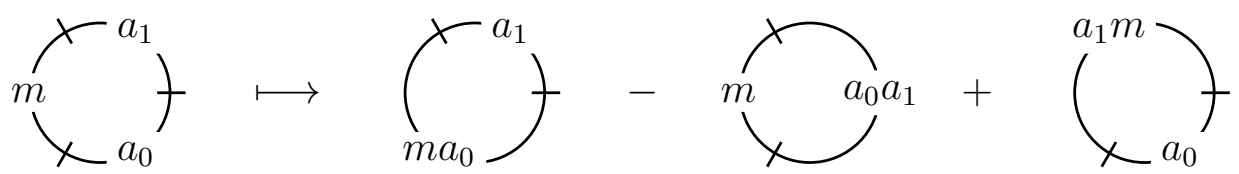

In the twisted case add a mark on the circle between segments labeled $m$ and $a_{n}$. To merge these two segments, one has to move the mark over $a_{n}$, acting upon it with $\varphi$ :

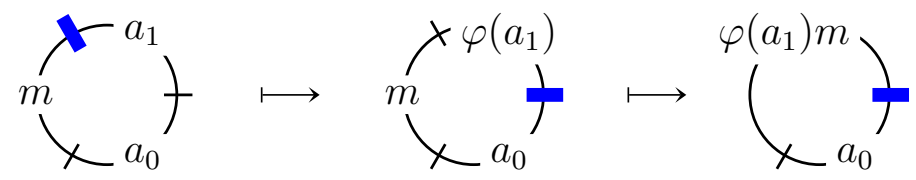

Defined a priori for the untwisted case, the cyclicity map (3.39) can be easily extended to the twisted homology following (3.34):

$$
\begin{aligned}
& C H_{\bullet}^{\psi}\left(B, \Sigma N^{\prime}{\underset{A}{\otimes}} N\right)=C H_{\bullet}\left(B,{ }_{\psi} B_{\psi} \underset{B}{\otimes} N^{\prime} \underset{A}{\otimes}{ }_{\varphi} N\right) \simeq \\
& C H_{\bullet}\left(A,{ }_{\varphi} N \underset{B}{\otimes} \psi_{\psi} B_{\psi} \underset{B}{\otimes} N^{\prime}\right) \cong C H_{\bullet}\left(A,{ }_{\varphi} N \underset{B}{\otimes} N^{\prime}\right)=C H_{\bullet}^{\varphi}\left(A, N \underset{B}{\otimes} N^{\prime}\right),
\end{aligned}
$$

which upgrades the twisted homology to a symmetric shadow on $\widetilde{\mathbf{R e p}}$. 


\subsubsection{Quantum Hochschild homology}

Fix an invertible elements $q \in \mathbb{k}$. We define the quantum Hochschild complex of a graded $(A, A)$-bimodule $M$ by replacing $\operatorname{coInv}(M)$ with its deformation:

$$
q C H_{\bullet}(A, M):=\operatorname{coInv}_{q}\left(M \underset{A}{\otimes} R_{\bullet}(A)\right) .
$$

It has the same chain groups as $C H \bullet(A, M)$ with coefficients extended to $\mathbb{k}^{\prime}$, but the last term of the differential is replaced with

$$
(-1)^{n} q^{-\left|a_{n}\right|} a_{n} m \otimes a_{1} \otimes \cdots \otimes a_{n-1} .
$$

The quantum Hochschild homology of $M$, denoted by $q H H_{\bullet}(A, M)$, is the homology of this complex.

The quantum Hochschild complex is a nonsymmetric shadow on gRep. Indeed, replacing the middle isomorphism in (3.39) with $\left[\underline{b} \otimes n^{\prime} \otimes \underline{a} \otimes n\right] \longmapsto q^{\left|\underline{b} \otimes n^{\prime}\right|}\left[\underline{a} \otimes n \otimes \underline{b} \otimes n^{\prime}\right]$ results in a homotopy equivalence

$$
\theta_{N^{\prime}, N}: q C H_{\bullet}\left(B, N^{\prime} \underset{A}{\otimes} N\right) \stackrel{\simeq}{\longrightarrow} q C H_{\bullet}\left(A, N \underset{B}{\otimes} N^{\prime}\right)
$$

that is no longer involutive: $\theta_{N, N^{\prime}} \circ \theta_{N^{\prime}, N}$ scales a chain of degree $d$ by $q^{d}$. The twisted Hochschild homology can be deformed likewise, leading to the twisted quantum Hochschild complex $q C H_{\bullet}^{\varphi}(A, M)$ of a graded $(A, A)$-bimodule $\mathrm{M}$ and its homology $q H H_{\bullet}^{\varphi}(A, M)$. The details are left to the reader.

\subsubsection{The functor $H H H$}

The construction of Hochschild homology extends naturally to complexes of bimodules, by letting $C H_{\bullet}\left(A, C^{\bullet}\right):=\operatorname{coInv}\left(C^{\bullet} \otimes_{A} R_{\bullet}(A)\right)$ for a complex of $(A, A)$-bimodules. It can be checked that it is a triangulated shadow on $\operatorname{Com}_{h}^{b}(\mathbf{R e p})$.

Instead of computing the total Hochschild homology of the complex $C^{\bullet}$ one can apply $H H$. component-wise. This leads to a Lefschetz shadow $H H H$ on $\mathrm{Com}_{/ h}^{b}(\mathbf{R e p})$ that replaces $C^{\bullet}$ with

$$
\ldots \longrightarrow H_{\bullet}\left(A, C^{i}\right) \longrightarrow H_{\bullet}\left(A, C^{i+1}\right) \longrightarrow H H_{\bullet}\left(A, C^{i+2}\right) \longrightarrow \ldots
$$

It is the second page of the spectral sequence associated to $\operatorname{coInv}\left(C^{\bullet} \otimes_{A} R \bullet(A)\right)$ seen as a bicomplex. This functor appears quite often in categorification of link invariants, when links are considered as closures of braids.

Naturally, when dealing with complexes of graded bimodules, one can replace $H H_{\bullet}$ with $q H H$. to obtain $q H H H$, which is a quantum shadow. According to Corollary 3.18, it restricts to a quantum trace on $\operatorname{Com}_{/ h}^{b}(\mathbf{g R e p})(A, B)$ for any graded algebras $A$ and $B$. It takes a particularly simple form when $A=B=\mathbb{k}$, because then $q H H_{0}(\mathbb{k})=\mathbb{k}$ and higher homology vanishes.

Corollary 3.22. Let $C^{\bullet} \in \operatorname{Com}_{/ h}^{b}(\mathbf{g R e p}(\mathbb{k}, \mathbb{k}))$ be a bounded complex and $f^{\bullet} \in \operatorname{End}\left(C^{\bullet}\right)$ its graded endomorphism. The endomorphism of $q H H(\mathbb{k}) \cong \mathbb{k}$ induced by $\left(C^{\bullet}, f^{\bullet}\right)$ is the multiplication by $\Lambda_{q}\left(f^{\bullet}\right)=\sum_{i, j}(-1)^{i} q^{j} \operatorname{tr}\left(f^{i, j}\right)$, where $i$ and $j$ are the homological and internal gradings in $C^{\bullet}$ respectively, and tr is the Hattori-Stallings trace on $\mathscr{R} e p(\mathbb{k})$. 


\subsubsection{Cobordisms of links in surface bundles}

It is generally a hard problem to identify the horizontal trace $h \operatorname{Tr}(\mathbf{C}, \Sigma)$ for a given bicategory $\mathbf{C}$, but the answer is very natural for $\operatorname{Tan}(F)$, the bicategory of points in a surface $F$, tangles in $F \times I$, and tangle cobordisms in $F \times I \times I$. Recall that the quotient space

$$
M:=F \times I /(p, 1) \sim(\varphi(p), 0),
$$

where $\varphi$ is a diffeomorphism of $F$, is a manifold called the surface bundle with fiber $F$ and monodromy $\varphi$.

Theorem A. Let $M$ be a surface bundle with fiber $F$ and monodromy $\phi \in \operatorname{Diff}(F)$. There is an equivalence of categories

$$
\mathrm{h} \operatorname{Tr}\left(\operatorname{Tan}(F), \phi_{*}\right) \simeq \mathscr{L i n k s}(M)
$$

where $\phi_{*}(S):=(\phi \times \operatorname{id} \times \mathrm{id})(S)$ for a cobordism $S \subset F \times I \times I$.

Proof. Let $\pi: F \times I \longrightarrow M$ be the quotient map and consider the fiber $F_{0}:=\pi(F \times\{0\})$ along which $M$ can be cut open to $F \times I$. Objects of $h \operatorname{Tr}\left(\operatorname{Tan}(F), \phi_{*}\right)$ can be identified with links in $M$ transverse to $F_{0}$, while morphisms are represented by link cobordisms in $M \times I$ transverse to the 3 -dimensional membrane $F_{0} \times I$. The cobordism can be deformed by an ambient isotopy that fixes the membrane, and the trace relation allows us to isotope the embedding of the membrane (although $F_{0} \times\{i\}$ is fixed for $i=0,1$ ).

There is an obvious functor $\operatorname{h} \operatorname{Tr}\left(\operatorname{Tan}(F), \phi_{*}\right) \longrightarrow \operatorname{Links}(M)$ that forgets the membrane. By the transversality argument it is essentially surjective on objects (each link is isotopic to a link transverse to $F_{0}$ ) and full on morphisms (each surface between links transverse to $F_{0}$ can be isotoped to be transverse to the standard membrane $\left.F_{0} \times I\right)$. It remains to show that if two surfaces $S, S^{\prime} \subset F \times I \times I$ represent isotopic cobordisms $\widehat{S}$ and $\widehat{S}^{\prime}$ in $M \times I$, then their images in $\operatorname{hTr}\left(\operatorname{Tan}(F), \phi_{*}\right)$ coincide.

Assume there is an isotopy $\varphi_{t}$ of $M \times I$ taking $\widehat{S}$ to $\widehat{S}^{\prime}$ with $\operatorname{support}{ }^{6} \operatorname{supp}(\varphi)$ disjoint from $M \times \partial I$ and a membrane $F^{\prime} \times I$ for some fiber $F^{\prime} \subset M$. It is enough to consider only such isotopies, because every two isotopic surfaces in $M \times I$ are connected by a sequence of them. If $F^{\prime}=F_{0}$, then $S$ and $S^{\prime}$ are already isotopic in $F \times I \times I$ and we are done. Otherwise, let $p \in \mathbb{S}^{1}$ be the point over which $F^{\prime}$ lives and consider a bump function $\beta: I \longrightarrow \mathbb{S}^{1}$ with $\beta(0)=\beta(1)=1$ and $\beta(t)=p$ for $t \in[\epsilon, 1-\epsilon]$ for some $\epsilon>0$ such that $\operatorname{supp}(\varphi) \subset M \times[\epsilon, 1-\epsilon]$, see Fig. 6. The preimage in $M \times I$ of the graph of $\beta$ is a membrane isotopic to $F_{0} \times I$; it can be visualized as pushing the interior of $F_{0} \times I$ onto $F^{\prime} \times I$. Because the new membrane is disjoint from the support of $\varphi$, the cuts of $\widehat{S}$ and $\widehat{S}^{\prime}$ along it are isotopic. This proves the faithfulness, because the cuts represent in $\mathrm{h} \operatorname{Tr}\left(\boldsymbol{T a n}(F), \phi_{*}\right)$ the same morphisms as the surfaces $S$ and $S^{\prime}$.

Each orientation preserving diffeomorphism $\phi$ of $\mathbb{R}^{2}$ is isotopic to identity, so that $\mathrm{h} \operatorname{Tr}\left(\operatorname{Tan}, \phi_{*}\right)$ is equivalent to the category of links in a solid torus. Because tangles in a thickened plane $\mathbb{R}^{2} \times I$ can be represented by diagrams on the stripe $\{0\} \times \mathbb{R} \times I$, is it worth to consider those diffeomorphisms that preserve the line $\{0\} \times \mathbb{R}$. There are two of them:

- the identity, in which case the stripe is closed to an annulus $\mathbb{A}$, and

- the rotation by 180 degrees, for which the image of the stripe is a Möbius band $\mathbb{M}$.

\footnotetext{
${ }^{6}$ A support of an isotopy is the closure of the set of points that are not stationary under the isotopy.
} 


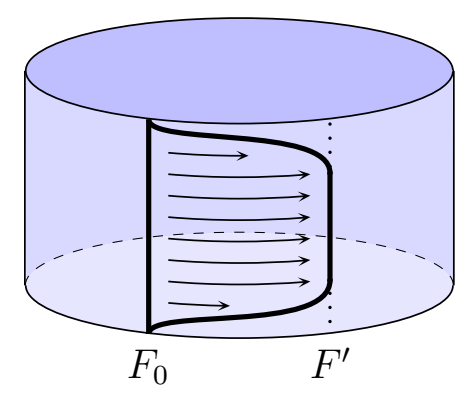

Figure 6: A visualization of the isotopy pushing $F_{0} \times I$ onto $F^{\prime} \times I$. Each point of the cylinder represents a fiber of the $F$-bundle $M \times I \longrightarrow \mathbb{S}^{1} \times I$. The thick straight line is the standard membrane $F_{0} \times I$, whereas the curve is its isotopic deformation. The flat part of the curve corresponds to the piece of the deformed membrane contained in $F^{\prime} \times I$.

The solid torus is a trivial line bundle over $\mathbb{A}$ and a twisted one over $\mathbb{M}$ respectively. Hence, given an invariant of tangles computed from their diagrams, there are two ways to get invariants of links in a solid torus.

Corollary 3.23. There are equivalences of categories

$$
\mathrm{h} \operatorname{Tr}(\operatorname{Tan}) \simeq \mathscr{L i n k s}\left(\mathbb{S}^{1} \times \mathbb{R}^{2}\right) \simeq \mathrm{h} \operatorname{Tr}\left(\operatorname{Tan}, \rho_{*}\right),
$$

where $\rho \in \operatorname{Diff}\left(\mathbb{R}^{2}\right)$ is the half-rotation. Thence, a bifunctor $\mathbf{I}:$ Tan $\longrightarrow \mathbf{C}$ induces invariants of links in a solid torus

$$
\begin{aligned}
\mathrm{h} \operatorname{Tr}(\mathbf{I}): \operatorname{Links}\left(\mathbb{R}^{2} \times \mathbb{S}^{1}\right) & \longrightarrow \mathrm{h} \operatorname{Tr}(\mathbf{C}), \text { and } \\
\mathrm{h} \operatorname{Tr}\left(\mathbf{I}, \rho_{*}\right): \mathscr{L i n k s}\left(\mathbb{R}^{2} \times \mathbb{S}^{1}\right) & \longrightarrow \mathrm{h} \operatorname{Tr}(\mathbf{C}, \Sigma),
\end{aligned}
$$

where $\Sigma$ is an endofunctor of $\mathbf{C}$ satisfying $\Sigma \circ \mathbf{I} \cong \mathbf{I} \circ \rho_{*}$. In particular, $\langle\langle\mathrm{h} \operatorname{Tr}(\mathbf{I})\rangle\rangle$ is a link invariant for any symmetric preshadow $\langle\langle-\rangle\rangle$ on $\mathbf{C}$.

\section{Quantum Hochschild homology}

Here we develop a machinery to show that higher quantum Hochschild homology of ChenKhovanov algebras vanish, which is used to proof the conjecture of Auroux, Grigsby, and Wehrli. In particular, we prove the invariance of quantum Hochschild homology under $K$-theoretic equivalences. This section can be skipped by a reader interested only in the construction of the quantum link homology. Hereafter we fix an invertible $q \in \mathbb{k}$.

\subsection{Twisted Hochschild-Mitchell homology}

Choose a small pregraded endocategory $(\mathscr{C}, \Sigma)$. The (twisted) quantum HochschildMitchell complex of $(\mathscr{C}, \Sigma)$ is the chain complex $q \mathscr{C} \mathscr{H}_{\bullet}(\mathscr{C}, \Sigma)$ with components

$$
q \mathscr{C} \mathscr{H}_{n}(\mathscr{C}, \Sigma):=\bigoplus_{x_{0}, \ldots, x_{n} \in \operatorname{Ob}(\mathscr{C})} \mathscr{C}\left(x_{0}, \Sigma x_{n}\right) \otimes \mathscr{C}\left(x_{1}, x_{0}\right) \otimes \cdots \otimes \mathscr{C}\left(x_{n}, x_{n-1}\right)
$$

and differential the alternating sum $\partial_{n}=\sum_{i=0}^{n}(-1)^{i} d_{n}^{i}$, where

$$
d_{n}^{i}\left(f_{0} \otimes \ldots \otimes f_{n}\right):= \begin{cases}f_{0} \otimes \ldots \otimes\left(f_{i} \circ f_{i+1}\right) \otimes \ldots \otimes f_{n} & \text { if } i<n, \\ q^{-\left|f_{n}\right|}\left(\Sigma f_{n} \circ f_{0}\right) \otimes f_{1} \otimes \ldots \otimes f_{n-1} & \text { if } i=n .\end{cases}
$$


Its homology is called the (twisted) quantum Hochschild-Mitchell homology of $(\mathscr{C}, \Sigma)$ and denoted by $q \mathscr{H} \mathscr{H} \cdot(\mathscr{C}, \Sigma)$. We also use the symbols $\mathscr{C} \mathscr{H} \bullet$ and $\mathscr{H} \mathscr{H} \bullet$ when $q=1$, dropping the adjective 'quantum'. In particular, $\mathscr{H} \mathscr{H} \bullet(\mathscr{C}, \mathrm{Id})$ recovers the usual HochschildMitchell homology of a category [Mit72]. Furthermore, $\mathscr{H} \mathscr{H}_{0}(\mathscr{C}, \Sigma)$ and $\mathscr{q} \mathscr{H}_{0}(\mathscr{C}, \Sigma)$ are precisely the universal $\Sigma$-twisted trace of $\mathscr{C}$ and its quantum deformation.

Expressing the differential as an alternating sum makes it evident that the HochschildMitchell complex arises from a presimplicial module [Lo, 1.0]. Despite not working in this framework, we will often use the following characterisation of chain homotopies.

Lemma 4.1. Choose a collection $\left\{h_{n}^{k}: q \mathscr{C H}_{n}(\mathscr{C}, \Sigma) \longrightarrow q \mathscr{C} \mathscr{H}_{n+1}\left(\mathscr{C}^{\prime}, \Sigma^{\prime}\right)\right\}_{0 \leqslant k \leqslant n}$ of linear maps, satisfying

$$
d_{n+1}^{i} \circ h_{n}^{k}= \begin{cases}h_{n-1}^{k-1} \circ d_{n}^{i} & \text { for } i<k, \\ d_{n+1}^{k} \circ h_{n}^{k} & \text { for } i=k+1, \\ h_{n-1}^{k} \circ d_{n}^{i-1} & \text { for } i>k+1 .\end{cases}
$$

Then the alternating sums $h_{n}:=\sum_{k=0}^{n}(-1)^{k} h_{n}^{k}$ are components of a chain homotopy from $f_{n}:=d_{n+1}^{0} \circ h_{n}^{0}$ to $g_{n}:=d_{n+1}^{n+1} \circ h_{n}^{n}$.

Given a morphism of endocategories $(F, \omega):(\mathscr{C}, \Sigma) \longrightarrow\left(\mathscr{C}^{\prime}, \Sigma^{\prime}\right)$ define a chain map $(F, \omega)_{*}: q \mathscr{C} \mathscr{H}_{\bullet}(\mathscr{C}, \Sigma) \longrightarrow q \mathscr{C} \mathscr{H}_{\bullet}\left(\mathscr{C}^{\prime}, \Sigma^{\prime}\right)$ by the formula

$$
(F, \omega)_{*}\left(f_{0} \otimes \ldots \otimes f_{n}\right):=\left(\omega \circ F f_{0}\right) \otimes F f_{1} \otimes \ldots \otimes F f_{n},
$$

where $\omega$ is used to fix the codomain of $F f_{0}$. It is straightfoward to check that the assignment $(F, \omega) \longmapsto(F, \omega)_{*}$ is functorial and it can be shown that $(F, \omega)_{*}$ and $\left(F^{\prime}, \omega^{\prime}\right)_{*}$ are chain homotopic if the morphisms are naturally isomorphic (this also follows from the results of the following section).

For a set of objects $B \subset \mathrm{Ob}(\mathscr{C})$ define a subcomplex $q \mathscr{C} \mathscr{H}_{\bullet}(\mathscr{C} \mid B, \Sigma) \subset q \mathscr{C} \mathscr{H}_{\bullet}(\mathscr{C}, \Sigma)$ by assuming that all $x_{i}$ in $(4.1)$ are from $B$. Recall that $B$ generates additively $\mathscr{C}$ in a graded sense if each object of $\mathscr{C}$ admits a homogeneous isomorphism, possibly of nonzero degree, to a direct summand of some $x_{1} \oplus \cdots \oplus x_{r}$ with $x_{i} \in B$.

Proposition 4.2. The inclusion $I: q \mathscr{C} \mathscr{H}_{\bullet}(\mathscr{C} \mid B, \Sigma) \longrightarrow q \mathscr{C} \mathscr{H}_{\bullet}(\mathscr{C}, \Sigma)$ is a homotopy equivalence if $B$ generates additively $\mathscr{C}$ in a graded sense. In particular, there are homotopy equivalences

$$
\begin{aligned}
& q \mathscr{C H} \cdot(\mathscr{C}, \Sigma) \stackrel{\simeq}{\longrightarrow} q \mathscr{C H} \cdot\left(\mathscr{C}^{\oplus}, \Sigma\right), \quad \text { and } \\
& q \mathscr{C H} \cdot(\mathscr{C}, \Sigma) \stackrel{\simeq}{\longrightarrow} \mathscr{C} \mathscr{H}_{\bullet}(\operatorname{Kar}(\mathscr{C}), \Sigma)
\end{aligned}
$$

induced by the canonical inclusions.

Proof. We proof the statement in two steps. First assume each object $x \in \mathscr{C}$ admits a decomposition $x=x_{1} \oplus \cdots \oplus x_{r}$ with $x_{i} \in B$, together with inclusions $\iota_{i}: x_{i} \longrightarrow x$ and projections $\pi_{i}: x \longrightarrow x_{i}$, both homogeneous of degree 0 . Pick the trivial decomposition if $x$ is already in $B$. This leads to a chain map $P: q \mathscr{C} \mathscr{H}_{\bullet}(\mathscr{C}, \Sigma) \longrightarrow q \mathscr{C} \mathscr{H} \cdot(\mathscr{C} \mid B, \Sigma)$

$$
P_{n}\left(f_{0} \otimes \cdots \otimes f_{n}\right):=\sum_{i_{0}, \ldots, i_{n}}\left(\Sigma \pi_{i_{n}} \circ f_{0} \circ \iota_{i_{0}}\right) \otimes\left(\pi_{i_{0}} \circ f_{1} \circ \iota_{i_{1}}\right) \otimes \cdots \otimes\left(\pi_{i_{n-1}} \circ f_{n} \circ \iota_{i_{n}}\right),
$$

which satisfies $P \circ I=\mathrm{id}$. To show that $I \circ P \simeq \mathrm{id}$, consider the family of maps 


$$
\begin{aligned}
h_{n}^{k}\left(f_{0} \otimes \cdots \otimes f_{n}\right):= & \sum_{i_{0}, \ldots, i_{k}}\left(f_{0} \circ \iota_{i_{0}}\right) \otimes\left(\pi_{i_{0}} \circ f_{1} \circ \iota_{i_{1}}\right) \otimes \cdots \\
& \otimes\left(\pi_{i_{k-1}} \circ f_{k} \circ \iota_{i_{k}}\right) \otimes \pi_{i_{k}} \otimes f_{k+1} \otimes \cdots \otimes f_{n}
\end{aligned}
$$

and apply Lemma 4.1. Indeed, one checks directly that $d_{n+1}^{0} \circ h_{n}^{0}=\mathrm{id}, d_{n+1}^{n} \circ h_{n}^{n}=I_{n} \circ P_{n}$, and the conditions (4.3) are satisfied.

Hence, by extending $B$, we can assume that for each object $x \in \mathscr{C}$ there is $b(x) \in B$ together with homogeneous morphisms $\iota_{x}: x \longrightarrow b(x)$ and $\pi_{x}: b(x) \longrightarrow x$, such that $\pi_{x} \circ \iota_{x}=\mathrm{id}_{x}$. Consider now the chain map $P: \mathscr{q}_{\mathscr{C}} \mathscr{H}_{\bullet}(\mathscr{C}, \Sigma) \longrightarrow q \mathscr{C} \mathscr{H}_{\bullet}(\mathscr{C} \mid B, \Sigma)$

$$
P_{n}\left(f_{0} \otimes \cdots \otimes f_{n}\right):=q^{\left|\iota_{n}\right|}\left(\Sigma \iota_{x_{n}} \circ f_{0} \circ \pi_{x_{0}}\right) \otimes\left(\iota_{x_{0}} \circ f_{1} \circ \pi_{x_{1}}\right) \otimes \cdots \otimes\left(\iota_{x_{n-1}} \circ f_{n} \circ \pi_{x_{n}}\right)
$$

where $x_{i}=\operatorname{dom}\left(f_{i}\right)$. Again, $P \circ I=\mathrm{id}$, whereas the other composition is chain homotopic to the identity by Lemma 4.1 applied to the collection of linear maps

$$
\begin{aligned}
h_{n}^{k}\left(f_{0} \otimes \cdots \otimes f_{n}\right):=\left(f_{0} \circ \pi_{x_{0}}\right) \otimes\left(\iota_{x_{0}} \circ f_{1} \circ \pi_{x_{1}}\right) \otimes \cdots & \\
& \otimes\left(\iota_{x_{i-1}} \circ f_{i} \circ \pi_{x_{i}}\right) \otimes \iota_{x_{i}} \otimes f_{i+1} \otimes \cdots \otimes f_{n} .
\end{aligned}
$$

Indeed, $\pi_{x} \circ \iota_{x}=\mathrm{id}_{x}$ implies that $d_{n+1}^{0} \circ h_{n}^{0}=\mathrm{id}$, and $d_{n+1}^{n+1} \circ h_{n}^{n}=I_{n} \circ P_{n}$ is immediate.

A graded $(A, A)$-bimodule $M$ can be understood as an endofunctor $(-) \otimes_{A} M$ on $\mathrm{g} \mathscr{R} e p(A)$ if it is finitely generated and projective as a right module. This leads to the identification of the quantum Hochschild-Mitchell homology of the representation category of $A$ with the quantum Hochschild homology of the algebra.

Corollary 4.3. Choose a graded algebra $A$ and a graded $(A, A)$-bimodule $M$ that is finitely generated and projective as a right module. Then the chain complexes $q C H \bullet$ and $q \mathscr{C H} .(\mathrm{g} \mathscr{R} e p(A), M)$ are homotopy equivalent.

Proof. The category gRep $(A)$ is generated additively by $B=\{A\}$ in a graded sense, where $A$ is seen as a right $A$-module. Therefore,

$$
q \mathscr{C} \mathscr{H}_{\bullet}(\mathrm{g} \mathscr{R e p}(A), M) \simeq q \mathscr{C} \mathscr{H}_{\bullet}(\mathrm{g} \mathscr{R} e p(A) \mid\{A\}, M) \cong q C H_{\bullet}(A, M)
$$

where the second isomorphism identifies $f \in \operatorname{End}_{A}(A)$ with $f(1) \in A$ and $g \in \operatorname{Hom}_{A}(A, M)$ with $g(1) \in M$.

Remark 4.4. Considering $\mathscr{R} e p(A)$ as a trivially graded category, the above result can be rephrased to say that $\mathscr{C} \mathscr{H}_{\bullet}(\mathscr{R e p}(A), M)$ is homotopy equivalent to $C_{\bullet}(A, M)$.

\subsection{Twisted Hochschild-Mitchell complex is a preshadow}

Let $\mathbb{k}$-gCat be the 2-category of pregraded linear categories. It is locally pregraded with a natural transformation $\eta$ being homogeneous of degree $d$ if each component $\eta_{x}$ is homogeneous of degree $d$. Objects of $\operatorname{hTr}_{q}(\mathbb{k}$-gCat $)$ are precisely pregraded endocategories. Recall that a morphism in the horizontal trace from $(\mathscr{C}, \Sigma)$ to $\left(\mathscr{C}^{\prime}, \Sigma^{\prime}\right)$ is represented by a pair $(F, \omega)$ consisting of a functor $F: \mathscr{C} \longrightarrow \mathscr{C}^{\prime}$ and a natural transformation $\omega: F \Sigma \longrightarrow \Sigma^{\prime} F$. Hence, $(F, \omega)$ is actually a functor of endocategories. It is then natural to ask, whether the chain map $(F, \omega)_{*}: q \mathscr{C} \mathscr{H}_{\bullet}(\mathscr{C}, \Sigma) \longrightarrow q \mathscr{C} \mathscr{H}_{\bullet}\left(\mathscr{C}^{\prime}, \Sigma^{\prime}\right)$ depends only on the image of the functor in the horizontal trace. 
Theorem 4.5. The quantum Hochschild-Mitchell complex descends to a functor

$$
q \mathscr{C H}: \operatorname{hTr}_{q}(\mathbb{k}-\text { gCat }) \longrightarrow \operatorname{Com}_{/ h}^{b}(\mathbb{k}) .
$$

In particular, it is a quantum preshadow on $\mathbb{k}$-gCat.

Proof. Consider the following two diagrams in $\mathbb{k}-\mathbf{g C a t}$
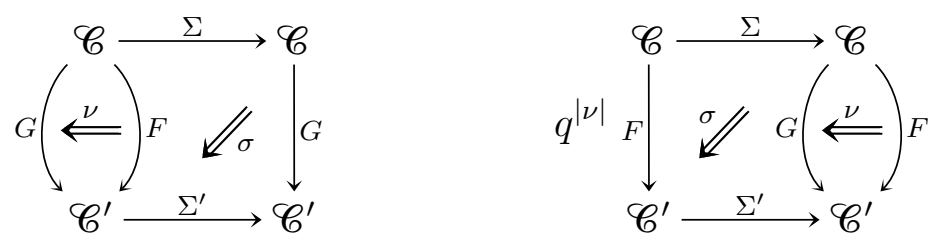

Composing the 2-morphisms results in morphisms $(G,(\nu \circ \mathbf{1}) * \sigma)$ and $\left(F, q^{|\nu|} \sigma *(\mathbf{1} \circ \nu)\right)$ of endocategories. We check directly that the family of maps

$$
h_{n}^{k}\left(f_{0} \otimes \ldots \otimes f_{n}\right):=q^{|\nu|}\left(\sigma_{x_{n}} \circ G f_{0}\right) \otimes G f_{1} \otimes \ldots \otimes G f_{k} \otimes \nu_{x_{k}} \otimes F f_{k+1} \otimes \ldots \otimes F f_{n}
$$

where $x_{i}=\operatorname{dom}\left(f_{i}\right)$, defines a chain homotopy between the induced chain maps. Indeed, $d_{n+1}^{0} \circ h_{n}^{0}=q^{|\nu|}(F, \sigma *(\mathbf{1} \circ \nu))_{n}$ by the naturality of $\nu$, whereas $d_{n+1}^{n+1} \circ h_{n}^{n}=(G,(\nu \circ \mathbf{1}) * \sigma)_{n}$ is straightforward.

Corollary 4.6. If $(F, \omega)$ and $\left(F^{\prime}, \omega^{\prime}\right)$ are isomorphic functors, then $(F, \omega)_{*}=\left(F^{\prime}, \omega^{\prime}\right)_{*}$.

Proof. Suppose $\eta:(F, \omega) \longrightarrow\left(F^{\prime}, \omega^{\prime}\right)$ is an isomorphism. Then

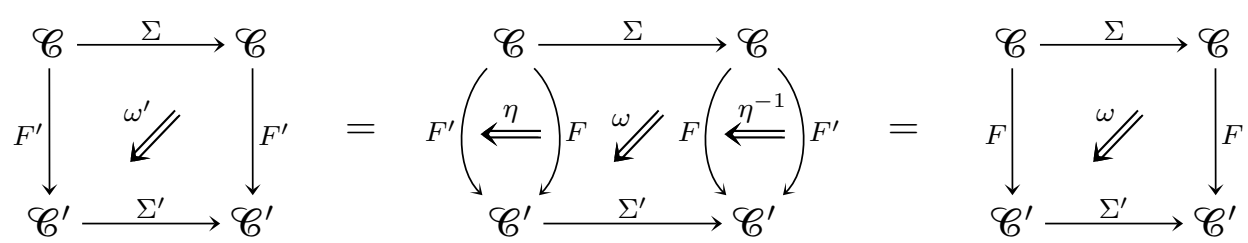

in $\operatorname{hrr}_{q}(\mathbb{k}$-gCat), where the second equality follows from the horizontal trace relation.

It follows from Theorem 4.5 that the quantum Hochschild-Mitchell homology is a preshadow on $\mathbb{k}$-gCat, which generalizes Example 3.8.1. In the view of Corollary 4.3 it induces a preshadow on $\mathbf{g R e p}$ with a priori a different cyclicity map.

Proposition 4.7. The homotopy equivalences $q \mathscr{C} \mathscr{H}_{\bullet}(\mathrm{g} \mathscr{R} e p(A), M) \simeq q C H \bullet(A, M)$ constitutes an isomorphism of preshadows.

Proof. We have to check that the square

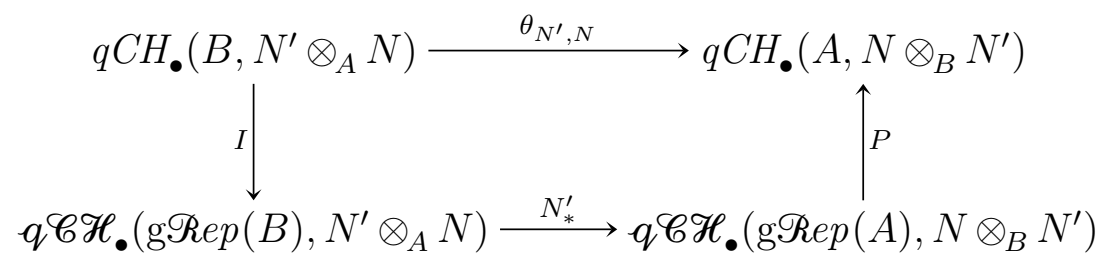

commutes up to a chain homotopy for bimodules $N \in \operatorname{gRep}(A, B)$ and $N^{\prime} \in \operatorname{gRep}(B, A)$, where $I$ and $P$ are mutually inverse homotopy equivalences. Due to the naturality of both $\theta_{N^{\prime}, N}$ and $N_{*}^{\prime}$ we can assume that $N^{\prime}$ is free as a right $A$-module with a graded basis 
$\left\{n_{1}^{\prime}, \ldots, n_{s}^{\prime}\right\}$. Then the left action of $B$ determines an algebra map $B \longrightarrow \operatorname{Mat}_{s}(A)$ that assigns to $b \in B$ the matrix $\left(a_{i j}(b)\right)_{1 \leqslant i, j \leqslant s}$ determined uniquely by the formula

$$
b n_{i}^{\prime}=\sum_{j} n_{j}^{\prime} a_{i j}(b)
$$

One checks that $a_{i j}\left(b^{\prime} b\right)=\sum_{k} a_{k j}\left(b^{\prime}\right) a_{i k}(b)$ for any $b, b^{\prime} \in B$. Likewise, $n_{k}^{\prime} \otimes n \otimes \mathrm{id}_{N^{\prime}}$ is represented, as a right $A$-linear map, by the matrix $\left(\delta_{j k} n \otimes n_{j}^{\prime}\right)_{1 \leqslant i, j \leqslant s}$. It follows from the proof of Proposition 4.2 that the composition $P \circ N_{*}^{\prime} \circ I$ is the chain map

$$
\left(n_{i_{0}}^{\prime} \otimes n\right) \otimes b_{r} \otimes \cdots \otimes b_{1} \longmapsto \sum_{i_{1}, \ldots, i_{r}=1}^{s} q^{\left|n_{i_{r}}^{\prime}\right|}\left(n \otimes n_{i_{r}}^{\prime}\right) \otimes a_{i_{r-1}, i_{r}}\left(b_{r}\right) \otimes \cdots \otimes a_{i_{0}, i_{1}}\left(b_{1}\right)
$$

To compute $\theta_{N^{\prime}, N}$, we need to lift the identity on $N^{\prime} \otimes_{A} N$ to a pair of graded chain maps

$$
p^{B A}: R_{\bullet}(B){\underset{B}{\otimes}}_{A} N^{\prime}{\underset{A}{\bullet}}_{\bullet}(A) \underset{A}{\otimes} N \rightleftarrows R_{\bullet}(B) \underset{B}{\otimes} N_{A}^{\prime}{\underset{A}{N}}^{\otimes} N: s^{B A} .
$$

By the uniqueness of a lift, $p^{B A}$ and $s^{B A}$ are mutually inverse homotopy equivalences.

The map $p^{B A}$ is defined as the standard projection on $R_{r}(B) \otimes_{B} N^{\prime} \otimes_{A} R_{0}(A) \otimes_{A} N=$ $R_{r}(B) \otimes_{N} N^{\prime} \otimes N$ and vanishes otherwise. The chain map $s^{B A}$ replaces each $b_{\ell}$, one-byone, with entries of the matrix $a_{i j}\left(b_{\ell}\right)$ :

$$
\begin{aligned}
s^{B A}\left(b_{r} \otimes \cdots \otimes\right. & \left.b_{1} \otimes\left(n_{i_{0}}^{\prime} \otimes n\right)\right):= \\
& \sum_{\ell=0}^{r} \sum_{i_{1}, \ldots, i_{\ell}=1}^{s} b_{r} \otimes \cdots \otimes b_{\ell+1} \otimes n_{i_{\ell}}^{\prime} \otimes a_{i_{\ell-1}, i_{\ell}}\left(b_{\ell}\right) \otimes \cdots \otimes a_{i_{0}, i_{1}}\left(b_{1}\right) \otimes n .
\end{aligned}
$$

The equality (4.5) makes $s^{B A}$ commute with the differential.

Up to the isomorphisms $\operatorname{coInv}_{q}\left(M \otimes_{A} R_{\bullet}(A)\right) \cong \operatorname{coInv}_{q}\left(R_{\bullet}(A) \otimes_{A} M\right)$ we can express $\theta_{N^{\prime}, N}$ as the composition $p^{A B} \circ \tau \circ s^{B A}$, where $\tau$ it the graded twist from 3.8.5. Because $\left|a_{i j}\left(b_{k}\right)\right|=\left|b_{k}\right|$ and $p^{A B}$ vanishes unless none of $b_{k}$ appears, the composition coincides with (4.6).

\section{3 $K$-theoretic invariance}

We say that a sequence of linear functors

$$
\left(F^{\prime}, \omega^{\prime}\right) \stackrel{\iota}{\longrightarrow}(F, \omega) \stackrel{\pi}{\longrightarrow}\left(F^{\prime \prime}, \omega^{\prime \prime}\right)
$$

semisplits if $0 \longrightarrow F^{\prime} x \stackrel{\iota_{x}}{\longrightarrow} F x \stackrel{\pi_{x}}{\longrightarrow} F^{\prime \prime} x \longrightarrow 0$ splits for every object $x$. Clearly, a split exact sequence semisplits, but not otherwise. Moreover, the sequence 4.8 remains semisplit when each of the three functors is pre- or post-composed with another functor, because linear functors preserve direct sums.

Let $\mathbf{g E n d o C a t}{ }^{\oplus}$ be the bicategory of small graded additive endocategories. We construct its semisplit Grothendieck category $K_{0}^{s s}$ (gEndoCat $\left.{ }^{\oplus}\right)$ by replacing functor categories with $\mathbb{Z}\left[q^{ \pm 1}\right]$-modules generated by isomorphism classes $[F, \omega]$ of functors modulo the relations $[F\{1\}, \omega\{1\}]=q[F, \omega]$ and $[F, \omega]=\left[F^{\prime}, \omega^{\prime}\right]+\left[F^{\prime \prime}, \omega^{\prime \prime}\right]$ for every semisplit exact sequence (4.8). The composition is well-defined by the discussion above. A functor $(F, \omega):(\mathscr{C}, \Sigma) \longrightarrow\left(\mathscr{C}^{\prime}, \Sigma^{\prime}\right)$ is a $K$-theoretic equivalence if it is an isomorphism in $K_{0}^{s s}\left(\right.$ gEndoCat $\left.^{\oplus}\right)$. In other words, there is a functor $\left(F^{\prime}, \omega^{\prime}\right):\left(\mathscr{C}^{\prime}, \Sigma^{\prime}\right) \longrightarrow(\mathscr{C}, \Sigma)$, called the $K$-theoretic inverse of $(F, \omega)$, such that the compositions $\left(F^{\prime}, \omega^{\prime}\right) \circ(F, \omega)$ and $(F, \omega) \circ\left(F^{\prime}, \omega^{\prime}\right)$ coincide in the corresponding Grothendieck groups with the images of the identity functors. 
Theorem 4.8 ( $K$-theoretic invariance). The homotopy class of the chain map $(F, \omega)_{*}$ assigned to a functor of linear graded endocategories $(F, \omega):(\mathscr{C}, \Sigma) \longrightarrow\left(\mathscr{C}^{\prime}, \Sigma^{\prime}\right)$ depends only on $[F, \omega] \in K_{0}^{s s}$ (gEndoCat $\left.{ }^{\oplus}\right)$. In particular, $(F, \omega)_{*}$ is a homotopy equivalence if the functor is a $K$-theoretic equivalence.

Proof. The relation $(F\{1\}, \omega\{1\})_{*}=q(F, \omega)_{*}$ follows from the proof of Proposition 4.2. Thence, we have to show that $(F, \omega)_{*}=\left(F^{\prime}, \omega^{\prime}\right)_{*}+\left(F^{\prime \prime}, \omega^{\prime \prime}\right)_{*}$ for a semisplit sequence of functors (4.8). For that fix an isomorphism $\varphi_{x}: F x \cong F^{\prime} x \oplus F^{\prime \prime} x$ for each $x \in \mathscr{C}$ that results from a splitting of the sequence $0 \longrightarrow F^{\prime} x \longrightarrow F x \longrightarrow F^{\prime \prime} x \longrightarrow 0$. Then every morphism $g \in \mathscr{C}(F x, F y)$ can be identified with a $2 \times 2$ matrix $\left(g_{i j}\right)$. In particular, the proof of Proposition 4.2 implies that $\eta_{*}$ is chain homotopic to

$$
f_{0} \otimes \ldots \otimes f_{n} \longmapsto \sum_{i_{0}, \ldots, i_{n}}\left(\omega \circ F f_{0}\right)_{i_{0}, i_{n}} \otimes\left(F f_{1}\right)_{i_{1}, i_{0}} \otimes \ldots \otimes\left(F f_{n}\right)_{i_{n}, i_{n-1}} .
$$

A simple diagram chasing reveals that for any $f \in \mathscr{C}(x, y)$ the matrix of $F f$ is upper triangular with $F^{\prime} f$ and $F^{\prime \prime} f$ on the diagonal, and likewise for $\omega_{x}$. Therefore, the only nontrivial summands in (4.9) are the two with $i_{0}=\cdots=i_{n}$, which are precisely $\left(F^{\prime}, \omega^{\prime}\right)_{*}\left(f_{0} \otimes \ldots \otimes f_{n}\right)$ and $\left(F^{\prime \prime}, \omega^{\prime \prime}\right)_{*}\left(f_{0} \otimes \ldots \otimes f_{n}\right)$.

\subsection{An application to Hochschild homology of algebras}

Let $A$ be a graded algebra and $\varphi \in \operatorname{Aut}(A)$ a graded automorphism. Following 2.4.6 construct the bimodule ${ }_{\varphi} A$ by redefining the left action as $a \cdot x:=\varphi(a) x$. The bimodules $A_{\varphi}$ and ${ }_{\varphi} A_{\varphi}$ are constructed likewise. The automorphism $\varphi$ can be seen as an isomorphism of bimodules $A \stackrel{\cong}{\longrightarrow} A_{\varphi} \cong{ }_{\varphi} A \otimes_{A} A_{\varphi}$. Tensoring with ${ }_{\varphi} A$ is an endofunctor on $\operatorname{g} \operatorname{Mod}(A)$ denoted in 2.4.6 by $(-)_{\bar{\varphi}}$. A module $V$ coincides with $V_{\bar{\varphi}}$, except that the action of $A$ is twisted: $v \cdot a=v \varphi^{-1}(a)$.

Let $B$ and $\psi \in \operatorname{Aut}(B)$ be another graded algebra together with a graded automorphism. Following 3.8.3 we put $\Sigma M:=A_{\varphi} \otimes_{A} M \otimes_{B}{ } B$ for a graded $(A, B)$-bimodule $M$. It coincides with $M$, except that $\varphi$ and $\psi$ are used to twist both actions. Suppose there is a bimodule homomorphism $\omega: M \longrightarrow \Sigma M$. Then there is a $B$-linear map

$$
V_{\bar{\varphi}} \underset{A}{\otimes} M \stackrel{\operatorname{id} \otimes \omega}{\longrightarrow} V_{\bar{\varphi}} \underset{A}{\otimes} \bar{\varphi} M_{\bar{\psi}} \stackrel{\cong}{\longrightarrow}\left(V{\underset{A}{\otimes}}^{\longrightarrow} M\right)_{\bar{\psi}}
$$

for any right $A$-module $V$, where the second map is the isomorphism ${ }_{\varphi} A_{\varphi} \cong A$. Hence, $(M, \omega)$ determines a functor of endocategories.

Let $g \mathscr{B} \operatorname{imod}_{0}(A, \varphi ; B, \psi)$ be the category with objects graded pairs $\left(M, \omega_{M}\right)$ as above and morphisms between $\left(M, \omega_{M}\right)$ and $\left(N, \omega_{N}\right)$ graded bimodule maps $f: M \longrightarrow N$ that intertwine the structure, i.e. $\omega_{N} \circ f=f \circ \omega_{M}$. One checks directly that $\omega_{M}(\operatorname{ker} f) \subset \operatorname{ker} f$ and $\omega_{N}(\operatorname{im} f) \subset \operatorname{im} f$, so that $\operatorname{g} \mathscr{B} \operatorname{imod}_{0}(A, \varphi ; B, \phi)$ has kernels and cokernels. Thence, it is an abelian category. Restricting bimodules to those that are finitely generated and projective as left modules picks the subcategory $\mathrm{g} \mathscr{R e} p_{0}(A, \varphi ; B, \psi)$. It is additive, but not abelian. Note that the categories are slightly different from morphism categories of g $\widetilde{B i m o d}$ and gRep from Section 3.8.3.

Following the usual convention we shall write $G_{0}(A, \varphi ; B, \psi)$ and $K_{0}(A, \varphi ; B, \psi)$ for the (exact) Grothendieck groups ${ }^{7}$ of $\operatorname{g}_{\mathscr{B}} \operatorname{imod}_{0}(A, \varphi ; B, \psi)$ and $\mathrm{g} \mathscr{R} e p_{0}(A, \varphi ; B, \psi)$ respectively. The inclusion of categories induces a linear map $K_{0}(A, \varphi ; B, \psi) \longrightarrow G_{0}(A, \varphi ; B, \psi)$,

\footnotetext{
7 That is the isomorphism class of a bimodule $[M]$ is identified with $\left[M^{\prime}\right]+\left[M^{\prime \prime}\right]$ whenever there is an exact sequence $0 \longrightarrow M^{\prime} \longrightarrow M \longrightarrow M^{\prime \prime} \longrightarrow 0$.
} 
which is neither surjective nor injective in general. Because not all exact sequence in gRep ${ }_{0}(A, \varphi ; B, \psi)$ splits, $K_{0}(A, \varphi ; B, \psi)$ is usually a proper quotient of the split Grothendieck group.

The tensor product $\left(M \otimes_{B} M^{\prime}, \omega \otimes_{B} \omega^{\prime}\right)$ of bimodules $(M, \omega) \in \operatorname{gRep}(A, \varphi ; B, \psi)$ and $\left(M^{\prime}, \omega^{\prime}\right) \in \mathrm{g} \mathscr{R} e p_{0}(B, \psi ; C, \rho)$, belongs to $\mathrm{g} \mathscr{R e p} p_{0}(A, \varphi ; C, \rho)$. Hence, the categories $\mathrm{g} \mathscr{R e p} p_{0}(A, \varphi ; B, \psi)$ constitute a bicategory. Moreover, tensoring with $(M, \omega)$ is exact. Indeed, exact sequences in $\mathrm{g} R e p_{0}(B, \varphi ; C, \rho)$ split when considered as sequence of left modules, which is enough to prove the exactness of $(M, \omega) \otimes_{B}(-)$, whereas the exactness of $(-) \otimes_{A}(M, \omega)$ follows from $M$ being projective as a left module. Hence, the Grothendieck groups $K_{0}(A, \varphi ; B, \psi)$ form a category as well. Following [Ke98] we say that $(M, \omega)$ is a $K$-theoretic equivalence if $[M, \omega]$ is invertible in this category. The following is an immediate translation of Theorem 4.8.

Proposition 4.9 (Algebraic $K$-theoretic invariance). The homotopy class of the chain map $(M, \omega)_{*}: q C H_{\bullet}^{\psi}(B) \longrightarrow q C H_{\bullet}^{\varphi}(A)$ induced by $(M, \omega) \in \mathrm{g} R e p_{0}(A, \varphi ; B, \psi)$ depends only on $[M, \omega] \in K_{0}(A, \varphi ; B, \psi)$. In particular, $(M, \omega)_{*}$ is a homotopy equivalence if $(M, \omega)$ is a $K$-theoretic equivalence.

Choose now a positively graded algebra $A=\bigoplus_{d \in \mathbb{N}} A_{d}$. The degree zero subalgebra $A_{0} \subset A$ is preserved by any graded automorphism $\varphi \in \operatorname{Aut}(A)$. Hence, the inclusion and projection induce chain maps $q \mathrm{CH}_{\bullet}^{\varphi_{0}}\left(A_{0}\right) \longrightarrow q \mathrm{CH}_{\bullet}^{\varphi}(A)$ and $q \mathrm{CH}_{\bullet}^{\varphi}(A) \longrightarrow q \mathrm{CH}_{\bullet}^{\varphi_{0}}\left(A_{0}\right)$ respectively, where $\varphi_{0} \in \operatorname{Aut}\left(A_{0}\right)$ is the restriction of $\varphi$. One of the compositions is clearly the identity map, but not the other.

These chain maps may not be homotopy equivalences. For instance, it is known that the algebra of dual numbers $\mathbb{k}[x] /\left(x^{2}\right)$, where $\operatorname{deg} x=2$, has unbounded Hochschild homology, whereas the homology of $\mathbb{k}$ is one-dimensional. The situation changes drastically when $A$ has finite global dimension: the inclusion $A_{0} \longrightarrow A$ induces an isomorphism on Hochschild homology under some other technical conditions [Ke98]. Here we reprove this result for the twisted homology.

Theorem 4.10. Let $\mathbb{k}$ be a field and $A=\bigoplus_{d \in \mathbb{N}} A_{d}$ a positively graded $\mathbb{k}$-algebra of finite dimension. If each simple $A$-module is one dimensional and $A$ has finite global dimension, then the inclusion $A_{0} \subset A$ induces a homotopy equivalence $q \mathrm{CH}_{\bullet}^{\varphi_{0}}\left(A_{0}\right) \longrightarrow q C \mathrm{H}_{\bullet}^{\varphi}(A)$ for any graded automorphism $\varphi \in \operatorname{Aut}(A)$.

Proof. The chain map $q \mathrm{CH}_{\bullet}^{\varphi_{0}}\left(A_{0}\right) \longrightarrow q C H_{\bullet}^{\varphi}(A)$ is induced by $(A, \varphi)$, seen as an object of $\mathrm{g} \mathscr{R} e p_{0}\left(A, \varphi ; A_{0}, \varphi_{0}\right)$. Likewise, the other chain map is induced by $\left(A_{0}, \varphi_{0}\right) \in$ $\mathrm{g} \mathscr{R} e p_{0}\left(A_{0}, \varphi_{0} ; A, \varphi\right)$, where the right action of $A$ on $A_{0}$ is given by the projection. A quick computation shows that $\left(A_{0}, \varphi_{0}\right) \otimes_{A}(A, \varphi) \cong\left(A_{0}, \varphi_{0}\right)$ in gRep $\left(A_{0}, \varphi_{0} ; A_{0}, \varphi_{0}\right)$. Hence, the composition $q \mathrm{CH}_{\bullet}^{\varphi_{0}}\left(A_{0}\right) \longrightarrow q \mathrm{CH}_{\bullet}^{\varphi}(A) \longrightarrow q \mathrm{CH}_{\bullet}^{\varphi_{0}}\left(A_{0}\right)$ is homotopic to the identity. In the view of Proposition 4.9 it is now enough to show that $(A, \varphi)$ and $(A, \varphi) \otimes_{A_{0}}\left(A_{0}, \varphi_{0}\right)$ coincide in $K_{0}(A, \varphi ; A, \varphi)$.

We check first that the bimodules have the same image in $G_{0}(A, \varphi ; A, \varphi)$. Indeed,

$$
\left.A_{\geqslant d} / A_{>d} \cong\left(A_{\geqslant d} \underset{A_{0}}{\otimes} A_{0}\right) / A_{>d} \underset{A_{0}}{\otimes} A_{0}\right)
$$

which shows that the graded associate of the bimodules coincide, and so their composition series (which are finite due to $A$ being of finite dimension). It remains to show that every $(A, A)$-bimodule of finite length can be represented in $K_{0}(A, \varphi ; A, \varphi)$. 
We first notice that $A^{e}:=A \otimes A^{o p}$ has finite global dimension: each simple $A^{e}$-module is of the form $L^{\prime} \otimes L^{*}$ for certain simple $A$-modules $L^{\prime}$ and $L$, and as such it has a uniformly bounded projective resolution. Thence, every $(A, A)$-bimodule $M$ of finite length has a finite projective resolution $P^{\bullet}(M)$. Because $A_{\varphi}$ and ${ }_{\varphi} A$ are projective as left and right modules, $\Sigma$ is exact and preserves projective bimodules. Hence, $P^{i}(\Sigma M):=\Sigma\left(P^{i}(M)\right)$ is a projective resolution of $\Sigma M$. Lifting $\omega$ to a chain map $P^{\bullet}(\omega)$ between these complexes, we construct a resolution of $(M, \omega)$ in $\mathrm{g} \mathscr{R} e p_{0}(A, \varphi ; A, \varphi)$. The assignment

$$
[M, \omega] \longmapsto \sum_{i}(-1)^{i}\left[P^{i}(M), P^{i}(\omega)\right]
$$

is then a two-sided inverse of $K_{0}(A, \varphi ; A, \varphi) \longrightarrow G_{0}(A, \varphi ; A, \varphi)$. Hence, the bimodules $(A, \varphi)$ and $(A, \varphi) \otimes_{A_{0}}\left(A_{0}, \varphi_{0}\right)$ coincide in $K_{0}(A, \varphi ; A, \varphi)$ as desired.

\section{Khovanov Homology}

With this section we move from the algebraic to the topological part of the paper. It starts with a brief description of the formal bracket, a very generic approach to Khovanovtype homology due to Bar-Natan [BN05], which is followed by a list of TQFT functors producing link homologies used in this paper. The main purpose of this section is to fix notation and conventions. In particular, the reader should be aware that our convention for the quantum grading makes Chen-Khovanov algebras negatively graded.

\subsection{The formal bracket}

Let us start with a brief reminder of the formal Khovanov bracket following [BN05]. In this section $F$ stands for a surface, possibly with boundary.

Choose a diagram $D \subset F$ of an oriented tangle $T \subset F \times \mathbb{R}$ and let $n$ stand for the number of crossings in $D$. To compute the formal Khovanov bracket of $T$ one begins with creating the $n$-dimensional cube of resolutions $\mathscr{I}(D)$ of $D$ defined as follows:

- each vertex $\xi$ of $\mathscr{F}(D)$ is decorated with the resolution $D_{\xi}$ of $D$, i.e. the collection of circles and proper intervals in $F$ obtained from $D$ by forgetting the orientation and replacing each $i$-th crossing $\lambda^{\prime}$ by its horizontal $\asymp$ or vertical $)$ ( smoothing for $\xi_{i}=0$ and $\xi_{i}=1$ respectively, and

- each edge is decorated by a cobordism with a unique saddle point over the smoothing being changed and directed from the resolution with less vertical smoothings to the one with more of them.

We can view $\mathscr{I}(D)$ as a commutative cubical diagram in $\mathscr{C o b}(F)$, the category with objects (non-oriented) flat tangles in $F$ and morphisms the isotopy classes of surfaces in $F \times I$. The degree of a surface $S \subset F \times I$ is given by the formula

$$
\operatorname{deg} S:=\chi(S)-\frac{\# B}{4}
$$

where $B$ is the set of corners and $\chi(S)$ the Euler characteristic of $S$. Alternatively, $\operatorname{deg} S$ counts the critical points of the natural height function $h: S \longrightarrow I$ with signs: a point of index $\mu$ contributes $(-1)^{\mu}$ towards deg. Thence, to ensure that each morphism in $\mathscr{I}(D)$ has degree 0 , we introduce formal degree $\operatorname{shifts}^{8}$ and place at each vertex $\xi$ the shifted resolution $D_{\xi}\{|\xi|\}$ for $|\xi|:=\xi_{1}+\cdots+\xi_{n}$.

\footnotetext{
${ }^{8}$ Formally, objects in $\mathscr{C o b}(F)$ are now symbols $T\{i\}$ formed by a flat tangle $T \subset F$ and $i \in \mathbb{Z}$.
} 
Let $\mathscr{C o b}(F)^{\oplus}$ be the additive closure of $\mathscr{C} o b(F)$. The formal Khovanov bracket $\llbracket D \rrbracket$ of the tangle diagram $D$ is the complex in $\mathscr{C} o b(F)^{\oplus}$ obtained from the cube by distributing signs over some edges to make every square anticommute, then taking direct sums along diagonal sections of the cube, and finally applying suitable degree shifts:

$$
\llbracket D \rrbracket^{i}:=\bigoplus_{|\xi|=i+n_{-}} D_{\xi}\left\{i+n_{+}-n_{-}\right\}
$$

where $n_{ \pm}$stand for the amount of positive or negative crossings in $D$.

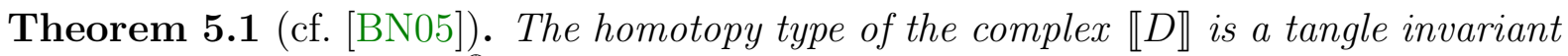
after imposing on $\operatorname{Cob}(F)^{\oplus}$ the following local relations ${ }^{9}$
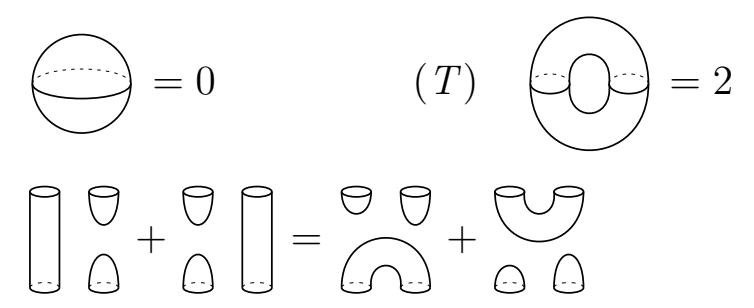

introduced first by Bar-Natan [BN05].

Let $\nwarrow, \lambda, \nearrow$, and $\asymp$ be tangle diagrams that coincide everywhere except a small disk, in which they look as indicated by the pictures. It follows from the construction that the formal brackets of the first two diagrams are mapping cones of chain maps between the formal complexes of the other two diagrams.

Proposition 5.2 (cp. [Kh99, BN05]). Let $\nwarrow, ~ \lambda, ~ \, \asymp$ be four link diagrams as above, where we choose any orientation for $\asymp$, and write $e=n_{-}(\asymp)-n_{-}(\supset\ulcorner)$. Then there are isomorphisms of formal complexes

$$
\begin{aligned}
& \llbracket \ltimes \rrbracket \cong \operatorname{cone}(\llbracket\rangle \backslash \rrbracket\{1\}[-1] \longrightarrow \llbracket \asymp \rrbracket\{3 e+2\}[-1-e]) \text {, and } \\
& \llbracket \lambda^{\top} \rrbracket \cong \operatorname{cone}(\llbracket \asymp \rrbracket\{3 e-2\}[-e] \longrightarrow \llbracket \backslash \backslash \backslash \rrbracket\{-1\}),
\end{aligned}
$$

where the chain maps are induced by saddle cobordisms.

The isomorphisms (5.3) and (5.4) were first observed in [Kh99, Section 4.2] for knots in $\mathbb{R}^{3}$ and then by Bar-Natan in the framework of the formal bracket [BN05, Lemma 4.4]. They lead to distinguished triangles in the homotopy category of $\mathscr{C o b}(F)^{\oplus}$, which can be seen as categorified versions of the Kauffman skein relation.

To retrieve homology groups from $\llbracket D \rrbracket$ one has to replace $\mathscr{C o b}(F)^{\oplus}$ with an abelian category. This is done by applying to the above construction a graded TQFT functor that preserves the relations $S, T$, and $4 T u$. Most known functors of this type factorize through the universal Bar-Natan skein category $\widetilde{\mathscr{B} \mathcal{N}}(F)$ [BN05], the additive linear category generated by circles and proper intervals in $F$ as objects, and formal linear combinations of cobordisms in $F \times I$ decorated by dots (each dot decreases the degree of a cobordism by 2) as morphisms, subject to the local relations

$$
(S) \bigoplus=0, \quad(D) \circlearrowright=1,
$$

\footnotetext{
${ }^{9}$ Locality means that each picture represents a part of a cobordism inside a ball in $F \times I$.
} 


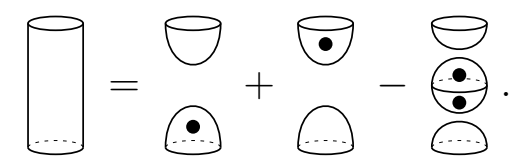

The last relation is commonly referred as the neck cutting relation and it allows to reduce any surface to a linear combination of surfaces of genus zero. Both $T$ and $4 T u$ follows, and a contractible circle can be replaced with a pair of shifted copies of an empty set. In particular, the empty set generates $\widetilde{\mathscr{B} \mathcal{N}}(F)$ when $F$ is either $\mathbb{R}^{2}$ or $\mathbb{S}^{2}$.

Proposition 5.3 (Delooping, cf. [BN07]). There is a pair of mutually inverse isomorphisms in $\widetilde{\mathscr{B N}}(F)$

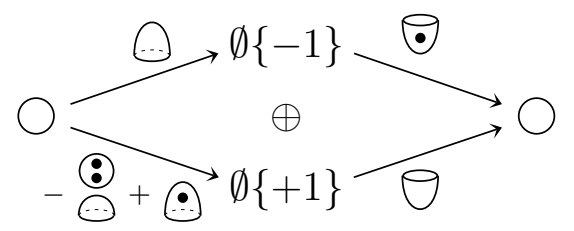

for every circle $\bigcirc$ bounding a disk in $F$.

The TQFT functors we consider in this paper factor through certain quotients of $\widetilde{\mathscr{B} \mathcal{N}}(F)$. Consider the following two relations:
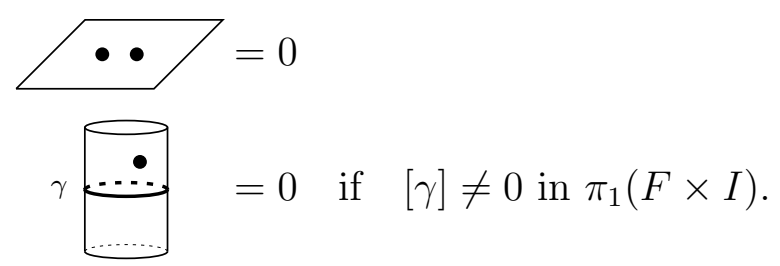

The first one asserts that two dots annihilate a cobordism when placed on the same connected component. Together with the neck cutting relation it implies further that a cobordism is annihilated when a dot is placed on its component of positive genus. The second relation prohibits a component of a cobordism to carry a dot if the component contains a closed curve that is nontrivial in $\pi_{1}(F \times I)$. In particular, a component of a cobordism cannot carry a dot if its boundary curves are not contractible in $F$.

We write $\mathscr{B} \mathcal{N}(F)$ when only $T D$ is imposed and $\mathscr{B} \mathscr{B} \mathcal{N}(F)$ when both. $\mathscr{B} \mathcal{N}(F)$ is commonly called the Bar-Natan skein category and it was first defined in [BN05]. The case of the annulus was extensively studied by Russell [Rus09]. The relation $B$ was introduced by Boerner [Boe08] for any surface $F$ and we call $\mathscr{B} \mathscr{B N}(F)$ the Boerner-Bar-Natan skein category.

\section{Functoriality}

The construction of the formal bracket $\llbracket T \rrbracket$ is functorial up to signs: given a cobordism $S$ between tangle diagrams $T$ and $T^{\prime}$ there is a chain map $\llbracket S \rrbracket: \llbracket T \rrbracket \longrightarrow \llbracket T^{\prime} \rrbracket$ defined up to the factor \pm 1 , such that $\llbracket S S^{\prime} \rrbracket= \pm \llbracket S \rrbracket \circ \llbracket S^{\prime} \rrbracket$. Hence, the same type of functoriality holds for any TQFT functor $\mathscr{F}: \mathscr{C o b}(F) \longrightarrow \mathscr{A}$ satisfying the relations $S, T$, and $4 T u$.

The chain map $\llbracket S \rrbracket$ is computed from a movie presentation of $S$, a sequence of generic sections $S_{t}=S \cap(F \times\{t\})$ called movie clips, such that the part $\left.S\right|_{\left[t, t^{\prime}\right]}$ of $S$ between two consecutive clips $S_{t}$ and $S_{t^{\prime}}$ is either one of the Reidemeister moves, a saddle cobordism, a cap, or a cup [CS98]. There is a well-defined chain map for each of the parts and $\llbracket S \rrbracket$ is defined as the composition of these pieces. A cobordism $S$ admits many movie presentations and it is proven that up to sign $\llbracket S \rrbracket$ does not depend on the presentation 
chosen [BN05]. Unfortunately, direct computation shows that the sign of $\llbracket S \rrbracket$ does depend on the presentation [Jac04].

There are a few approaches to attack the sign issue. In case $F=\mathbb{R}^{2}$ one can use the Lee deformation of the Khovanov homology [Le05] to define canonical generators, which are preserved by $\llbracket S \rrbracket$ up to sign $[\operatorname{Ras} 05]$. We can then redefine $\llbracket S \rrbracket$ so that the generators are actually preserved. This approach was used in [GLW15] to fix signs in certain cases.

A different idea is to replace $\mathscr{C} o b(F)$ with another category. This was done successfully for $F=\mathbb{R}^{2}$ by Clark, Morrison and Walker using cobordisms with seams and coefficients in the ring of Gaussian integers $\mathbb{Z}[i]$ [CMW09], then by Blanchet using nodal foams [Bla10] and by Vogel using mixed cobordisms: locally oriented cobordisms with certain disorientation curves [Vo15]. The first strictly functorial construction for $(2 m, 2 n)$-tangles is due to Caprau [Ca07], and is defined over Gaussian integers. It assigns to a tangle a direct summand of the corresponding invariant due to Chen and Khovanov, which is used in this paper. In a forthcoming paper [BHPW18] we address the functoriality of the ChenKhovanov invariant by rephrasing it in terms of $\mathfrak{g l}_{2}$ foams and constructing an explicit isomorphism between the new and the original invariant. Finally, the functoriality of the bracket for links in thickened surfaces has been fixed in a recent work of Queffelec and Wedrich [QW18], who constructed certain foam categories that extend the nodal foams introduced by Blanchet.

\subsection{Khovanov homology for links in $\mathbb{R}^{3}$}

Let $R$ be a commutative algebra and $A$ a Frobenius algebra over $R$ of rank 2. This datum determines a TQFT functor with $\mathscr{F}(\emptyset):=R$ and $\mathscr{F}(\bigcirc):=A$, and it produces an invariant chain complex for link diagrams on a plane [Kh06]. For instance, Khovanov's functor $\mathscr{F}_{K h}: \mathscr{C o b}\left(\mathbb{R}^{2}\right) \longrightarrow \operatorname{Mod}(\mathbb{k})$ is defined this way by taking $R:=\mathbb{k}$ and equipping $A:=R w_{+} \oplus R w_{-}$with the structure maps

$$
\begin{array}{ll}
m: A \otimes A \longrightarrow A \longrightarrow A \otimes A & \left\{\begin{array}{l}
w_{+} \otimes w_{+} \longmapsto w_{+}, \\
w_{ \pm} \otimes w_{\mp} \longmapsto w_{-}, \\
w_{-} \otimes w_{-} \longmapsto 0,
\end{array}\right. \\
\Delta: A \longrightarrow w_{+} \otimes w_{-}+w_{-} \otimes w_{+}, \\
\eta: \mathbb{k} \longrightarrow A & \left\{\begin{array}{l}
w_{+} \longmapsto w_{+} \\
w_{-} \longmapsto w_{-} \otimes w_{-},
\end{array}\right. \\
\epsilon: A \longrightarrow \mathbb{k} & \left\{\begin{array}{l}
1 \longmapsto w_{+}, \\
w_{+} \longmapsto 0, \\
w_{-} \longmapsto 1 .
\end{array}\right.
\end{array}
$$

The functor is graded if we set $\operatorname{deg} w_{ \pm}:= \pm 1$. We shall write $K h(D)$ for the homology of $C K h(D):=\mathscr{F}_{K h} \llbracket D \rrbracket$, where $D$ is a diagram of a link $L$; it is called the Khovanov homology of the link $L$.

Khovanov's functor factorizes through $\mathscr{B} \mathcal{N}\left(\mathbb{R}^{2}\right)$, where a dot is understood as multiplication by $w_{-}$. In particular, both generators are images of $1 \in \mathbb{k}$ under cup cobordisms

$$
\mathscr{F}_{K h}(\circlearrowleft): 1 \longmapsto w_{+} \quad \mathscr{F}_{K h}(\bullet): 1 \longmapsto w_{-}
$$

which motivates the following graphical description of $\mathscr{F}_{K h}$. Given a collection of curves $\Gamma \subset \mathbb{R}^{2}$ we identify $\mathscr{F}_{K h}(\Gamma)$ with the module generated freely by all diagrams obtained 
from $\Gamma$ by decorating some curves with dots and imposing the relation that two dots on a single curve annihilate the diagram. ${ }^{10}$ For example,

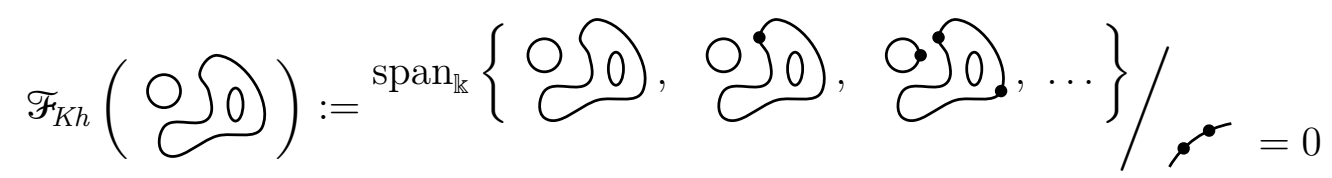

The generators $w_{+}$and $w_{-}$of the algebra $A$ are represented by the circle without and with a dot respectively. To redefine $\mathscr{F}_{K h}$ on a cobordism $S$ we use the following rules:

- if $S$ creates a circle, then $\mathscr{F}_{K h}(S)$ modifies a diagram by inserting the new circle with no dot on it,

- if $S$ contracts a circle, then $\mathscr{F}_{K h}(S)$ removes the circle from a diagram if it was decorated by a dot, or takes the diagram to 0 otherwise, and

- we use the following local surgery formulas to define $\mathscr{F}_{K h}(S)$ if $S$ is a merge or a split

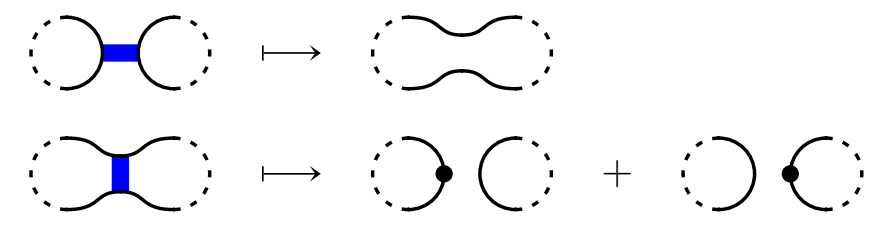

where the blue thick arcs visualize the saddle of $S$.

Notice that a merge of two curves is zero, when each curve carries a dot, as the surgery (5.11) produces a curve with two dots. Likewise, a split of a curve with a dot results in one diagram, as one of the two terms at the right hand side of (5.12) vanishes.

\subsection{Annular link homology}

There are two types of closed curves in the annulus: trivial curves bounding disks in $\mathbb{A}$, and essential curves, parallel to the core of $\mathbb{A}$. The value of an annular TQFT functor $\mathscr{F}: \mathscr{B N}(\mathbb{A}) \longrightarrow \operatorname{Mlod}(\mathbb{k})$ on trivial curves is determined by Bar-Natan's relations, but not the value on essential curves.

The first construction of an annular TQFT functor is due to Asaeda, Przytycki, and Sikora [APS04]. The APS functor $\mathscr{F}_{\mathbb{A}}: \mathscr{B N}(\mathbb{A}) \longrightarrow \operatorname{Mod}(\mathbb{k})$ assigns to a trivial and an essential curve the free modules

$$
W:=\operatorname{span}_{\mathbb{k}}\left\{w_{+}, w_{-}\right\}, \quad V:=\operatorname{span}_{\mathbb{R}}\left\{v_{+}, v_{-}\right\},
$$

respectively, with the degree defined on generators as

$$
\operatorname{deg} w_{ \pm}= \pm 1, \quad \operatorname{deg} v_{ \pm}=0
$$

This degree is denoted by $j^{\prime}$ in [GLW15] and differs from the one used in [Rob13]. In addition, the modules admit the annular grading, denoted adeg and defined as

$$
\operatorname{adeg} w_{ \pm}=0, \quad \operatorname{adeg} v_{ \pm}= \pm 1 .
$$

One can define $\mathscr{F}_{\mathbb{A}}$ by comparing it to $\mathscr{F}_{K h}$. Indeed, $V$ and $W$ are isomorphic as ungraded modules, but (5.14) induces a filtration on $\mathscr{F}_{K h}$ and the functor $\mathscr{F}_{\mathbb{A}}$ can be constructed as the graded associate [Rob13]. For completeness we write down the maps corresponding to the elementary saddle moves. A merge is assigned one of the maps

\footnotetext{
${ }^{10}$ Clearly, those diagrams in which each circle carries at most one dot form a free basis for $\mathscr{F}_{K h}(\Gamma)$.
} 


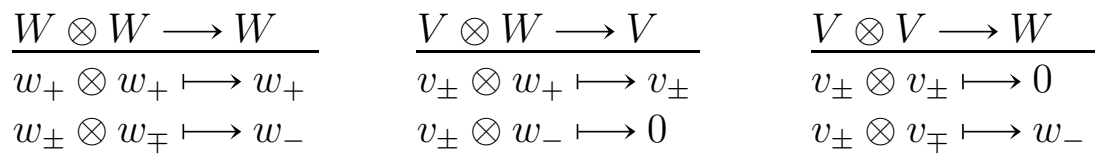

$$
\begin{aligned}
& w_{-} \otimes w_{-} \longmapsto 0
\end{aligned}
$$

depending on the curves involved, whereas for splits we choose one of

$$
\begin{array}{lll}
\frac{W \longrightarrow W \otimes W}{w_{-} \longmapsto w_{-} \otimes w_{-}} & \frac{V \longrightarrow V \otimes W}{v_{ \pm} \longmapsto v_{ \pm} \otimes w_{-}} & \begin{array}{l}
W \longrightarrow V \otimes V \\
w_{+} \longmapsto w_{+} \otimes w_{-}+w_{-} \otimes w_{+}
\end{array}
\end{array}
$$

The value of $\mathscr{F}_{\mathbb{A}}$ on caps and cups is unchanged.

The graphical description of $\mathscr{F}_{K h}$ can be extended to the annular case. Trivial curves can again carry dots, but the essential ones cannot, because the merge cobordism takes $v_{ \pm} \otimes w_{-}$to zero. Therefore, we shall visualize the two generators of $V$ by choosing an orientation of the essential curve, anticlockwise for $v_{+}$and clockwise for $v_{-}$:

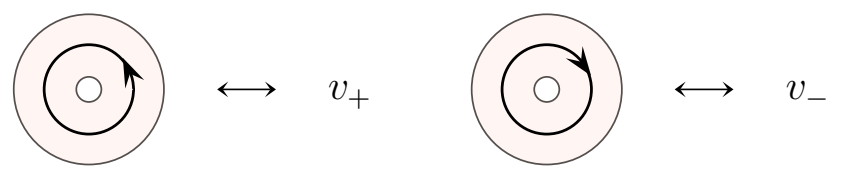

We use the usual surgery formulas for merging a trivial curve to an essential one or splitting it off, keeping in mind that an essential curve cannot carry dots:
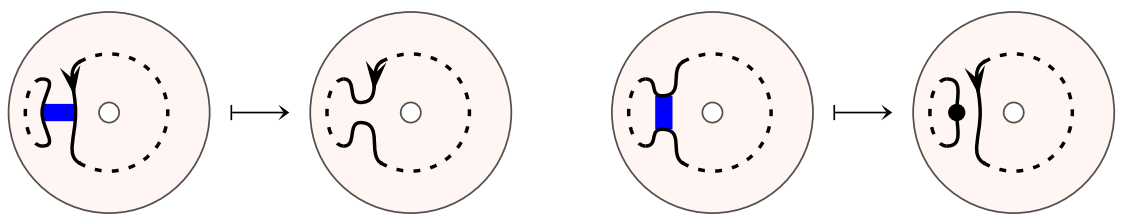

Two essential curves can be merged together only if they have opposite orientations, in which case we decorate the resulting trivial curve with a dot, and otherwise we have zero:
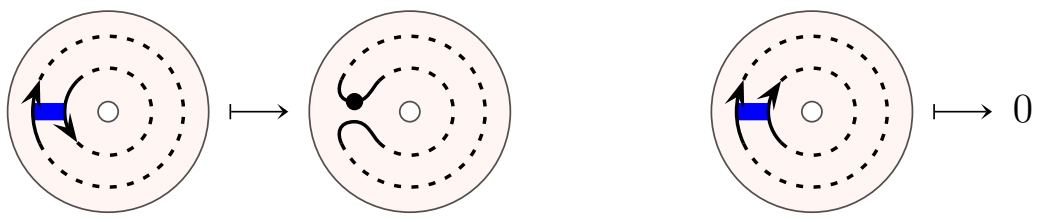

Finally, a surgery from a trivial curve to two essential ones is assigned the map

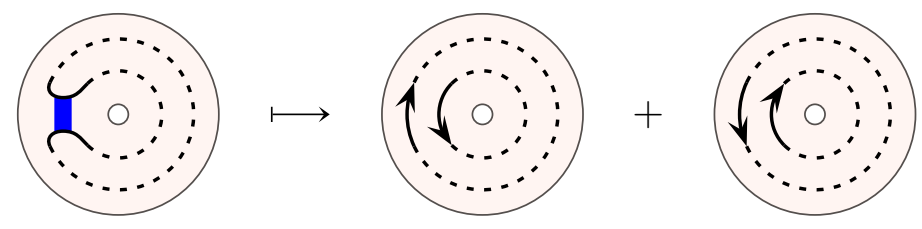

which can be viewed as performing the surgery on the trivial curve considered with both orientations at the same time.

\section{Action of $\mathfrak{s l}_{2}$}

It has been recently observed in [GLW15] that the annular link homology admits an action of $\mathfrak{s l}_{2}$ if we consider $W$ as a trivial representation and $V$ is identified with the fundamental one $V_{1}=\operatorname{span}_{\mathbb{k}}\left\{v_{+}, v_{-}\right\}$or its dual $V_{1}^{*}=\operatorname{span}_{\mathbb{k}}\left\{v_{+}^{*}, v_{-}^{*}\right\}$, depending on the nestedness of 
the associated essential curve, i.e. $V_{1}$ and $V_{1}^{*}$ are assigned alternatively. The following tables describe the action of $\mathfrak{s l}_{2}$.

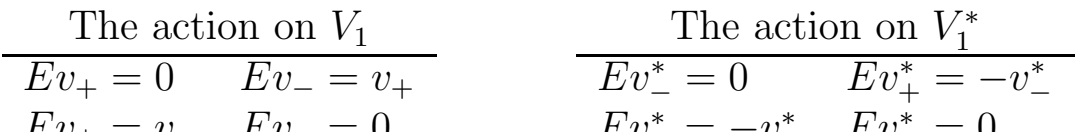

$$
\begin{aligned}
& F v_{+}=v_{-} \quad F v_{-}=0 \quad F v_{-}^{*}=-v_{+}^{*} \quad F v_{+}^{*}=0
\end{aligned}
$$

There is an obvious isomorphism of $\mathfrak{s l}_{2}$-modules $V_{1} \cong V_{1}^{*}$, which identifies $v_{ \pm}$with $\pm v_{\mp}^{*}$. However, the action on the annular chain complex is defined using instead the linear isomorphism $V \cong V_{1}^{*}$ that sends $v_{ \pm}$to $v_{\mp}^{*}$, so that the action depends on the position of $V$ in the tensor product. For instance, two essential curves are assigned $V \otimes V \cong V_{1}^{*} \otimes V_{1}$ with $\mathfrak{s l}_{2}$ acting in the following way:

$$
\begin{array}{ll}
E\left(v_{+} \otimes v_{+}\right)=0 & F\left(v_{+} \otimes v_{+}\right)=v_{-} \otimes v_{+}-v_{+} \otimes v_{-} \\
E\left(v_{+} \otimes v_{-}\right)=-v_{+} \otimes v_{+} & F\left(v_{+} \otimes v_{-}\right)=v_{-} \otimes v_{-} \\
E\left(v_{-} \otimes v_{+}\right)=v_{+} \otimes v_{+} & F\left(v_{-} \otimes v_{+}\right)=-v_{-} \otimes v_{-} \\
E\left(v_{-} \otimes v_{-}\right)=v_{+} \otimes v_{-} v_{-} \otimes v_{+} & F\left(v_{-} \otimes v_{-}\right)=0
\end{array}
$$

It follows that the maps $V \otimes V \longrightarrow W$ and $W \longrightarrow V \otimes V$ intertwine the action and the annular TQFT functor is upgraded to $\mathscr{F}_{\mathbb{A}}: \mathscr{B} \mathcal{N}(\mathbb{A}) \longrightarrow \mathrm{g} \mathscr{R e p}\left(\mathfrak{s l}_{2}\right)$. In particular, $\mathfrak{s l}_{2}$ acts on the triply graded annular homology.

Remark. The action admits the following graphical description: each clockwise oriented curve in a diagram $w$ contributes to $E w$ a diagram obtained from $w$ by reversing the curve, and scaling it by $(-1)$ if it is separated from the outer boundary by an odd number of curves. Likewise for $F$ we reverse orientations of anticlockwise oriented curves.

\subsection{Homology for links in a thickened Möbius band}

Beyond links in a thickened annulus, we also consider links in the twisted line bundle over the Möbius band ("twisted" means that the monodromy along the orientation reversing curve is $-i d$, so that the bundle is an orientable 3 -manifold). Let us recall the construction of the APS functor in this case.

A Möbius band $\mathbb{M}$ admits three types of curves: trivial curves bounding disks, separating curves cutting an annulus out of $\mathbb{M}$, and nonseparating ones. The APS functor $\mathscr{F}_{\mathbb{M}}: \mathscr{B N}(\mathbb{M}) \longrightarrow \operatorname{Mod}(\mathbb{k})$ assigns to them the following free modules

$$
W:=\operatorname{span}_{\mathbb{k}}\left\{w_{+}, w_{-}\right\}, \quad V:=\operatorname{span}_{\mathbb{k}}\left\{v_{+}, v_{-}\right\}, \quad \text { and } \quad U:=\operatorname{span}_{\mathbb{k}}\left\{u_{+}, u_{-}\right\},
$$

with the degree function vanishing on both $V$ and $U$, and $\operatorname{deg} w_{ \pm}= \pm 1$ as usual. There are more types of saddle cobordisms in $\mathbb{M} \times I$ than in $\mathbb{A} \times I$, and $\mathscr{F}_{\mathbb{M}}$ vanishes on those without trivial circles in the boundary. Otherwise, it is defined as in the annular case for merges and splits (where both $V$ and $U$ can play the role of the "annular" $V$ ) and one of the maps

$$
\begin{aligned}
& \frac{W \longrightarrow V}{w_{+} \longmapsto v_{+}+v_{-}} \\
& w_{-} \longmapsto 0
\end{aligned} \quad \begin{aligned}
& \frac{V \longrightarrow W}{v_{ \pm} \longmapsto w_{-}} \\
&
\end{aligned}
$$


for a saddle cobordism between a trivial circle and a separating one. We can represent the latter graphically as

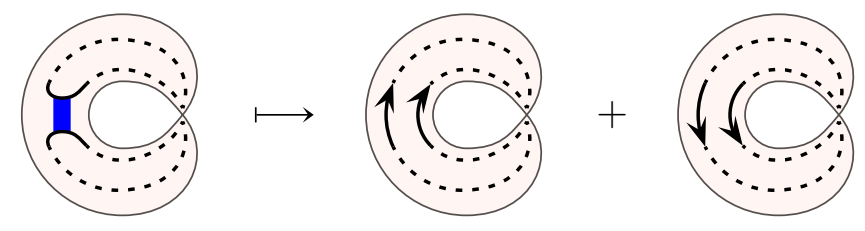

and

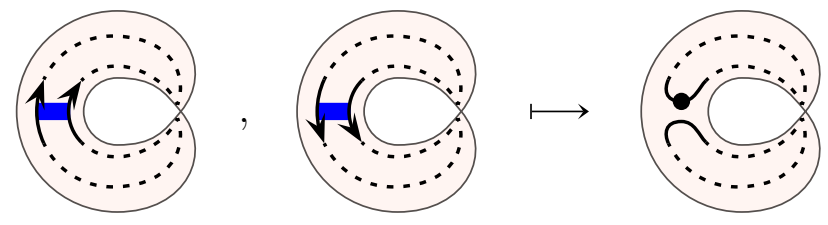

We shall write $C K h_{\mathbb{M}}(D):=\mathscr{F}_{\mathbb{M}} \llbracket D \rrbracket$ for the chain complex for a link diagram $D$ on $\mathbb{M}$, and $K h_{\mathbb{M}}(D)$ for its homology. A resolution of $D$ can have at most one nonseparating curve such a curve cuts the band into an annulus. In particular, $U$ only appears in $C K h_{\mathbb{M}}(D)$ when $D$ meets any cross section of $\mathbb{M}$ in an odd number points. If so, $C K h_{\mathbb{M}}(D) \cong \overline{C K h}_{\mathbb{M}}(D) \otimes U$, where we write $\overline{C K h}_{\mathbb{M}}(D)$ for the chain complex of $D$ computed with $\mathbb{k}$ assigned to non-separating curves instead of $U$.

\subsection{Chen-Khovanov homology for tangles}

Tangle diagrams in a thickened stripe $\mathbb{R} \times I$ form a category. Thence, the formal Khovanov bracket of a tangle is a chain complex built over the bicategory $\operatorname{Cob}=\mathscr{C o b}(\mathbb{R} \times I)$ of points on a line, flat tangles in a stripe $\mathbb{R} \times I$, and surfaces in $(\mathbb{R} \times I) \times I$. This bicategory is graded with the degree of a surface $S$ defined in (5.1). To preserve this richer structure, the homology for tangles is constructed by Chen and Khovanov [CK14] using a 2-functor $\mathbf{F}_{C K}$ : $\mathbf{C o b} \longrightarrow$ gBirep valued in the bicategory of graded bimodules that are sweet, i.e. finitely generated and projective as left and as right modules, but not necessarily as bimodules. In fact, $\mathbf{F}_{C K}$ factors through the Bar-Natan skein bicategory $\mathbf{B N}=\mathscr{B} \mathcal{N}(\mathbb{R} \times I)$. We begin with describing the modules assigned to tangles, then the algebras assigned to points, and finally reconstructing the bimodule structure.

\section{Cup diagrams with platforms}

A crossingless matching between $2 n$ points in a line is a collection of $n$ disjoint arcs attached to the points. We shall draw the arcs in the lower half-plane $\mathbb{R} \times \mathbb{R}_{-}$and refer to them as a cup diagram. Following [CK14] we generalize cup diagrams to allow semiinfinite arcs, each attached to one point only and going left or right towards infinity. This can be visualized by drawing two vertical platforms going out of the horizontal line, one to the left and one to the right of all the points, and attaching semi-infinite arcs to them. In particular, odd number of points are allowed. We shall call the points on the line termini to distinguish them from the endpoints on the platforms. Figure 7 presents all cup diagrams with three termini.

Let $\mathscr{G} M^{n}$ be the set of such diagrams with $n$ termini. We define the weight of a diagram $a \in \mathscr{G M}^{n}$ as $\operatorname{wt}(a):=r-\ell$, where $r$ and $\ell$ count respectively the arcs terminating on the right and on the left platform. In what follows we shall write $\mathscr{G} \mathcal{M}^{n}(\lambda) \subset \mathscr{G} \boldsymbol{M}^{n}$ for 

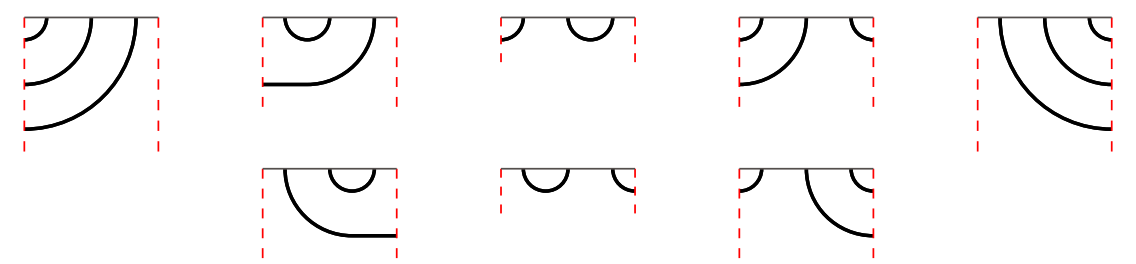

Figure 7: The generalized cup diagrams with three termini.

the subset of diagrams of weight $\lambda$. Notice that $\mathscr{G} \mathcal{M}^{n}(\lambda)$ is empty unless $\lambda$ has the same parity as $n$.

Dually we define the set $\mathscr{G M}_{n}$ of cap diagrams with platforms with arcs drawn in the upper half-plane. The reflection along the horizontal line induces a bijection of sets

$$
\mathscr{G M}^{n}(\lambda) \ni a \longmapsto a^{!} \in \mathscr{G}_{n}(\lambda)
$$

for every $n$ and $\lambda$.

\section{An extension of $\mathscr{F}_{K h}$}

A pair of cup diagrams $a \in \mathscr{G} \boldsymbol{M}^{m}(\lambda)$ and $b \in \mathscr{G} \boldsymbol{M}^{n}(\lambda)$ can be used to produce a planar closure $b^{!} T a$ of any flat tangle $T \in \mathbf{C o b}(m, n)$. The closure is constructed by gluing $a$ to the bottom of $T$ and $b$ ! to the top, then turning the platforms towards themselves and identifying the endpoints of the arcs from inside out. In case $m \neq n$ there will be unmatched endpoints, the same number at each side, because $a$ and $b$ have equal weights. We connect them with half-circles, see Figure 8.

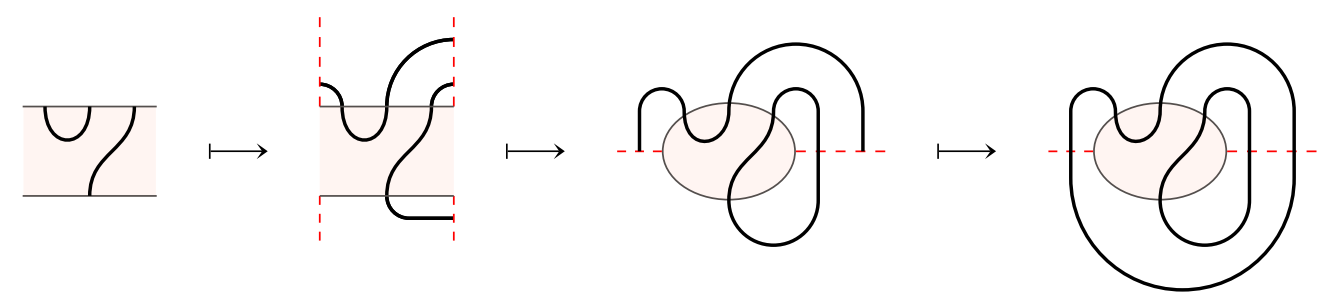

Figure 8: The construction of a planar closure of a (1,3)-tangle.

The functor $\mathscr{F}_{K h}: \mathscr{C o b}\left(\mathbb{R}^{2}\right) \longrightarrow \operatorname{Mod}(\mathbb{k})$ is extended to collections of curves with platforms by assigning to such a collection a module generated by all possible decorations of the curves with dots as before, but with more restrictions:

1) a diagram vanishes when it contains a curve intersecting any of the platforms twice,

2) a dot annihilates a diagram when placed on a curve that intersects a platform, and

3) as before, two dots on one curve annihilate the diagram.

A diagram is nonadmissible if one of the above situations happens, see Figure 9. Surgeries (5.11) and (5.12) on nonadmissible diagrams produce nonadmissible ones, so that $\mathscr{F}_{K h}(S)$ is well-defined for any surface $S$, see also [CK14]. The Chen-Khovanov functor assigns to a flat tangle $T \in \mathbf{C o b}(m, n)$ the module

$$
\mathbf{F}_{C K}(T):=\bigoplus_{\lambda} \mathbf{F}_{C K}(T ; \lambda), \quad \text { with } \quad \mathbf{F}_{C K}(T ; \lambda):=\bigoplus_{\substack{a \in \mathscr{G}, M^{m}(\lambda) \\ b \in \mathscr{G}, M^{n}(\lambda)}} \mathscr{F}_{K h}(b ! T a),
$$


and to a cobordism $S$ between flat tangles $T_{0}$ and $T_{1}$ the linear map

$$
\mathbf{F}_{C K}(S):=\bigoplus_{\lambda} \mathbf{F}_{C K}(S ; \lambda) \text {, with } \quad \mathbf{F}_{C K}(S ; \lambda):=\bigoplus_{\substack{a \in \mathscr{G}, M^{m}(\lambda) \\ b \in \mathscr{G}, M^{n}(\lambda)}} \mathscr{F}_{K h}(b ! S a),
$$

where $b ! S a$ stands for the surface $(b ! \times I) \cup S \cup(a \times I)$.
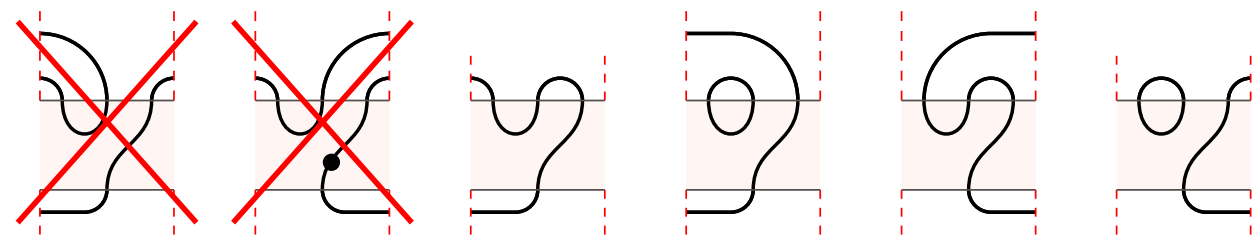

Figure 9: Examples of generators of $\mathbf{F}_{C K}(\cup /)$. The first two diagrams are nonadmissible, because they contain either a turnback or a dot on an open arc.

Remark. The closures of $\cup /$ in Figure 9 are drawn without identifying the platforms of cup diagrams. Not only makes this smaller diagrams, but also easier to describe the module structure on $\mathbf{F}_{C K}(T)$ once the Chen-Khovanov algebras are introduced.

Example 5.4. Consider the saddle cobordism $S:=\mathbb{H}:|| \longrightarrow \stackrel{\smile}{\frown}$ between the identity $(2,2)$-tangle and the tangle consisting of a cap followed by a cup. The module $\mathbf{F}_{C K}(||)$ has seven generators, on which $\mathbf{F}_{C K}(S)$ takes the following values:
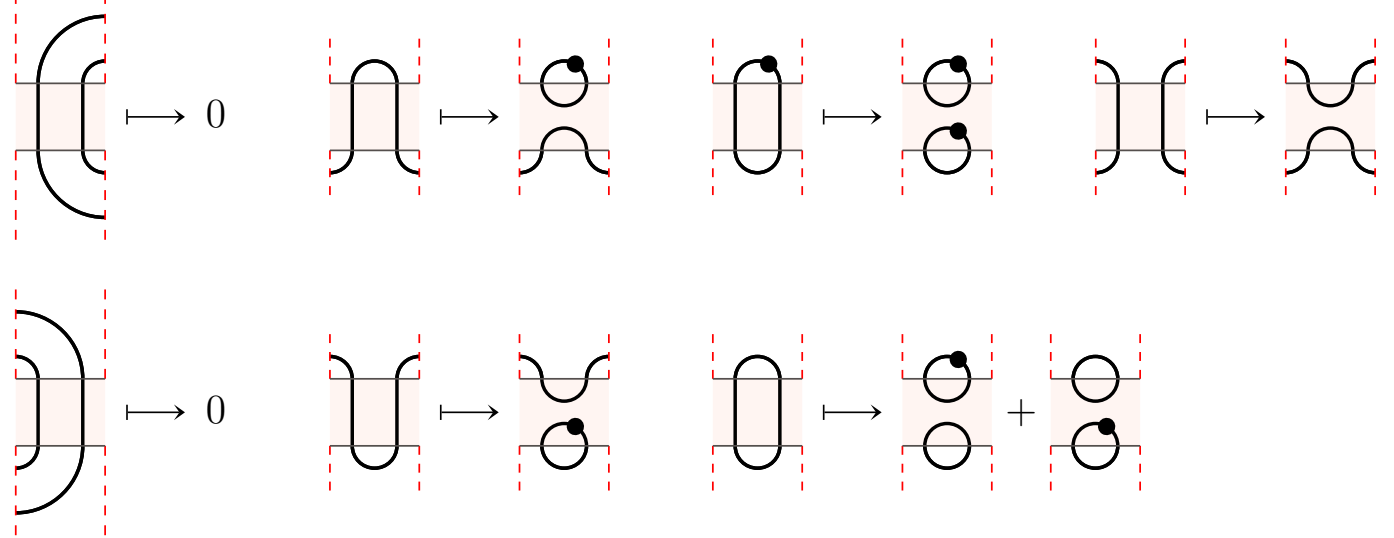

For example, the top right component of $\mathbf{F}_{C K}(S)$ is a merge when the platforms are identified:

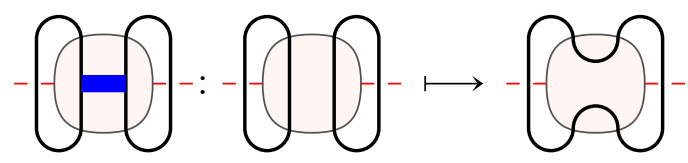

Example 5.5. Consider now the cobordism $S:=\breve{Y}: \stackrel{\smile}{\smile} \longrightarrow||$ going in the other direction. The module $\mathbf{F}_{C K}(\underset{\smile}{\smile})$ has eight generators, on which $\mathbf{F}_{C K}(S)$ is defined as below:
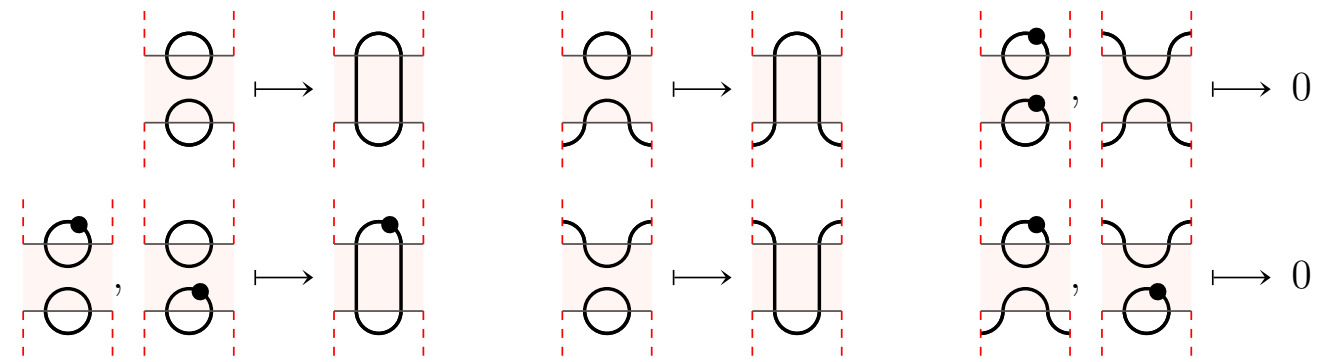
The two arcs in the top right corner are mapped to zero, because the corresponding closure of $S$ in this case is a split with each circle in its output touching a platform:

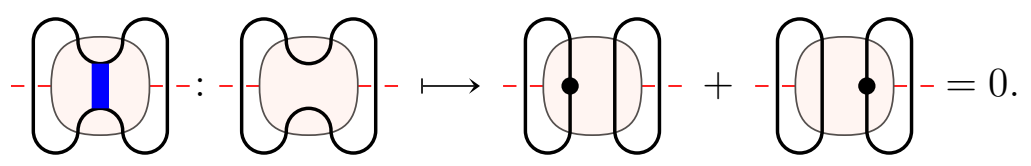

\section{Arc algebras and diagrammatic bimodules}

Let $c \in \mathscr{G} \boldsymbol{M}^{n}$ be a generalized cup diagram and write $S_{c}$ for the cobordism from $c \sqcup c^{!}$to $2 n$ vertical lines obtained by a sequence of $n$ surgeries, one per arc in $c$, see Figure 10. The collection of such cobordisms defines linear maps

$$
\mu_{T^{\prime}, T}: \mathbf{F}_{C K}(T) \otimes \mathbf{F}_{C K}\left(T^{\prime}\right) \longrightarrow \mathbf{F}_{C K}\left(T^{\prime} T\right)
$$

one per a pair of tangles $T \in \operatorname{Cob}(m, n)$ and $T^{\prime} \in \operatorname{Cob}(n, k)$. Explicitly, $\mu_{T^{\prime}, T}(x \otimes y)=0$ for $x \in \mathbf{F}_{C K}\left(b^{!} T a\right)$ and $y \in \mathbf{F}_{C K}\left(d^{!} T^{\prime} c\right)$ unless $b=c$, in which case $\mu_{T^{\prime}, T}=\mathscr{F}_{K h}\left(d^{!} T^{!} S_{c} T a\right)$.

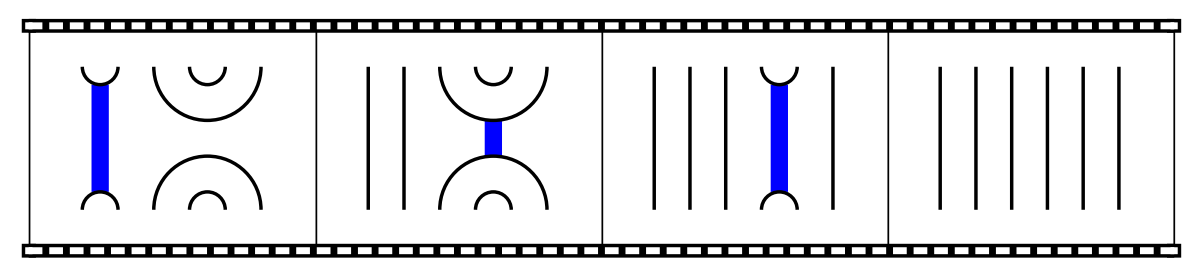

Figure 10: A sequence of surgeries replacing a disjoint union of a cup diagram and its vertical flip with vertical lines.

The Chen-Khovanov algebra $A^{n}$ is the module assigned to the tangle formed by $n$ vertical lines, with $x \cdot y:=\mu(x \otimes y)$. It admits a weight decomposition

$$
A^{n}=\bigoplus_{\lambda} A^{n}(\lambda)
$$

which is related to that from [CK14] by setting $A^{n-k, k}=A^{n}(n-2 k)$. There is a unique primitive idempotent $e_{c} \in A^{n}$ for each closure $c \in \mathscr{G} \mathbb{M}^{n}$ given by the diagram $c^{!} c$ with no dots. The idempotents are mutually orthogonal, and their sum is a unit in $A^{n}$.

Example 5.6. The algebra $A^{2}$ has generators in weights $-2,0$, and 2. Both $A^{2}(-2)$ and $A^{2}(2)$ are one dimensional, whereas $A^{2}(0)$ has five generators:

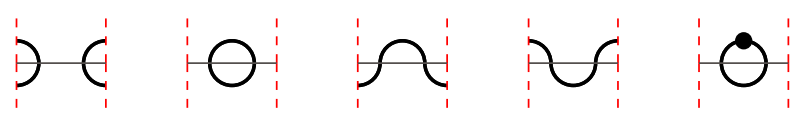

of which the first two are idempotents and the other square to zero. Furthermore,

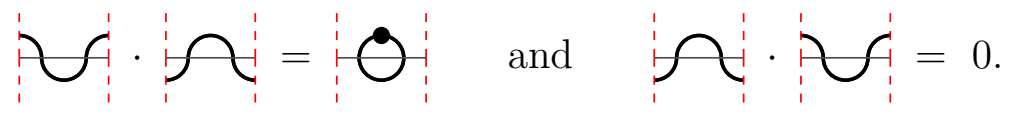

The product in $A^{n}$ can be described explicitly using generalized surgeries as in [BS11]. Because we do not identify platforms when drawing diagrams, each diagram has four platforms drawn vertically. The product $x \cdot y$, when nonzero, can be then computed graphically by placing $y$ on $x$, connecting the platforms in between, and following the two steps below. 
Step I: surgeries at platforms. Replace two opposite arcs touching one of the platforms with a vertical line and decorate with a dot each closed loop created that way:
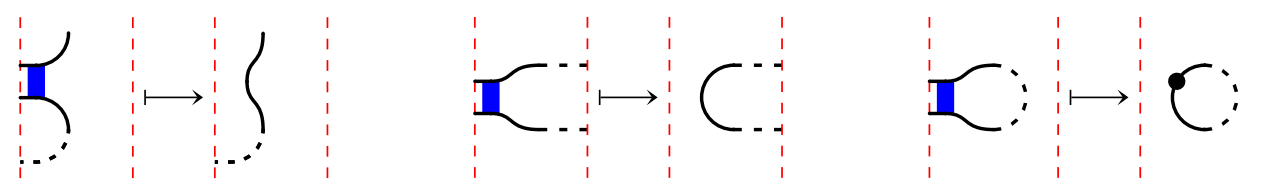

Step II: surgeries on half-circles. When no arc at inner platforms is left, perform surgeries on the remaining arcs using the usual surgery formulas (5.11) and (5.12), except that a merge of two open arcs is zero and a diagram with a dot on an open arc vanishes (in particular, the first term in the result of the second surgery may vanish):
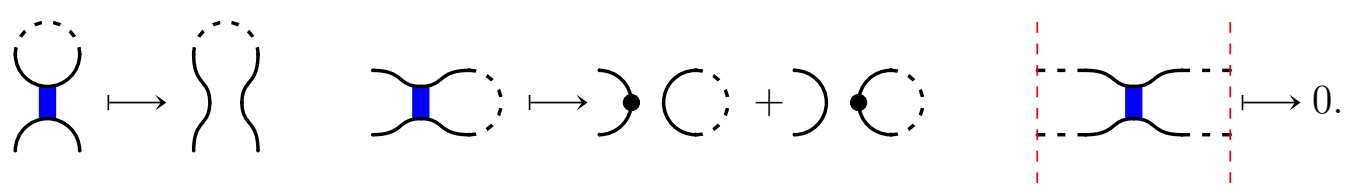

The merge of two arcs vanishes in the second step, because the two arcs have endpoints on the outer platforms and they belong to the same circle when the platforms are identified (compare with (5.27)).

It follows from the construction that the maps $\mu_{T^{\prime}, T}$ are natural with respect to tangle cobordisms. Furthermore, (5.26) can be extended to dotted surfaces, because $\mathbf{F}_{C K}$ is defined by $\mathscr{F}_{K h}$, in which case $\mathbf{F}_{C K}(S)=0$ if any component of $S$ carries two dots. Thence, the following result holds, see also [CK14].

Proposition 5.7. $\mathbf{F}_{C K}(T)$ is a sweet $\left(A^{n}, A^{m}\right)$-bimodule for any flat tangle $(m, n)$-tangle $T$, where the actions of the algebras are given by $\mu$. Moreover, (5.28) descend to natural isomorphisms of bimodules

$$
\mathbf{F}_{C K}(T) \underset{A^{n}}{\otimes} \mathbf{F}_{C K}\left(T^{\prime}\right) \stackrel{\cong}{\longrightarrow} \mathbf{F}_{C K}\left(T^{\prime} T\right)
$$

so that there is a strong bifunctor $\mathbf{F}_{C K}: \mathbf{B N} \longrightarrow$ Birep. $^{11}$

Throughout the paper we call $\mathbf{F}_{C K}(T)$ a diagrammatic bimodule. They are called geometric in [CK14]. Each $\mathbf{F}_{C K}(T)$ has a two sided dual $\mathbf{F}_{C K}\left(T^{!}\right)$, where $T^{!}$is the vertical flip of $T$. It is also known that each weight component $\mathbf{F}_{C K}(T ; \lambda)$ is indecomposable when $T$ contains no loops [BS10, Theorem 4.14], and otherwise $\mathbf{F}_{C K}(T ; \lambda) \cong \mathbf{F}_{C K}(\widetilde{T} ; \lambda) \otimes \mathbb{k}^{2 \ell}$, where $\widetilde{T}$ is the tangle $T$ with $\ell$ loops removed. Therefore, the category of diagrammatic bimodules has duals and is closed under direct summands.

\section{Grading}

The grading on $A^{n}$ is defined in [CK14] by shifting by $n$ the grading induced by the functor $\mathscr{F}_{K h}$. This does not work well for bimodules assigned to tangles, though. For instance, regarding a cup diagram $c \in \mathscr{G} \mathcal{M}^{n}$ as a flat $(0, n)$-tangle, there is an isomorphism of graded bimodules $\mathbf{F}_{C K}(c) \otimes \mathbf{F}_{C K}\left(c^{!}\right) \cong e_{c} A^{n} e_{c}$, but $e_{c} A^{n} e_{c}$ is graded differently from $\mathbf{F}_{C K}\left(c^{!} c\right)$.

In [BS11, BS10] a grading is computed differently. It agrees with that from [CK14] for arc algebras, and it is coherent with tensor products of tangle bimodules. On the other

\footnotetext{
${ }^{11}$ Recall that $M \circ N:=N \otimes M$ in Birep.
} 
hand, it depends on a Morse decomposition of a tangle, although it is well understood how the degree changes under planar isotopies, see [BS10, Lemma 2.4]. This motivates the following definition.

Let $T$ be a flat tangle with $\ell$ loops and $c$ arcs connecting bottom endpoints. Given a diagram $x \in \mathbf{F}_{C K}(T)$ orient all its curves counterclockwise. The platforms and boundary lines of $T$ split some curves of $x$ into vertical lines, caps, and cups. Let $a$ be the number of cups and caps with clockwise orientation. Then the degree of $x$ is given by the formula,

$$
\operatorname{deg} x:=\ell+c-a-2 d,
$$

where $d$ is the number of dots. For example,

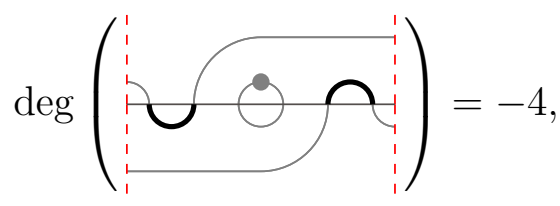

and

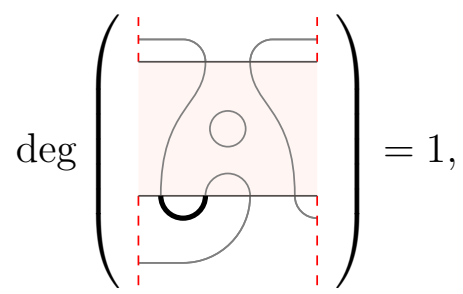

where the arcs with clockwise orientation are thickened. A quick look on the surgery formulas (5.11) and (5.12) reveals that for any tangle cobordism $S$ the map $\mathbf{F}_{C K}(S)$ is homogeneous of degree deg $S$. Furthermore, $\mu_{T^{\prime}, T}: \mathbf{F}_{C K}(T) \otimes \mathbf{F}_{C K}\left(T^{\prime}\right) \longrightarrow \mathbf{F}_{C K}\left(T^{\prime} T\right)$ preserves the degree, see [BS10, Theorem 3.5 (iii)], so that (5.34) is an isomorphism of graded bimodules. However, $\mathbf{F}_{C K}\left(T^{!}\right)$is dual to $\mathbf{F}_{C K}(T)$ only up to a degree shift.

\section{Chain complex and homology}

Assume now that $T$ is an oriented tangle with $m$ points at the bottom and $n$ at the top. The Chen-Khovanov complex $C_{C K}(T):=\mathbf{F}_{C K} \llbracket T \rrbracket$ is a chain complex of graded sweet $\left(A^{m}, A^{n}\right)$-bimodules, obtained from the formal bracket by applying the bifunctor $\mathbf{F}_{C K}$ component-wise. We refer to the homology $K h^{\bullet}(T):=H_{\bullet}\left(C_{C K}(T)\right)$ as the Chen-Khovanov homology. It is a triply graded theory: beyond the homological and quantum grading $K h^{\bullet}(T)$ admits a weight decomposition.

\section{Decategorification}

Let $V$ be the fundamental representation of $\boldsymbol{U}_{q}\left(\mathfrak{s l}_{2}\right)$. It is known that flat tangles can be intrepreted as intertwiners between tensor powers of $V$, see Appendix A.1. More precisely, there is a linear Temperly-Lieb category $\mathscr{T} \mathscr{L}$, the morphisms of which are generated by flat tangles, and a functor $\mathscr{F}_{T L}: \mathscr{T} \mathscr{L} \longrightarrow \mathscr{R} e p\left(\boldsymbol{U}_{q}\left(\mathfrak{s l}_{2}\right)\right)$ that takes a collection of $n$ points to $V^{\otimes n}$, whereas a cap and a cup to the evaluation and coevalution map (A.6) respectively. The Chen-Khovanov construction categorifies this functor.

Theorem 5.8 (cf. [CK14]). There are isomorphisms $\gamma_{n}: K_{0}\left(A^{n}\right) \otimes_{\mathbb{Z}\left[q^{ \pm 1}\right]} \mathbb{k} \cong V^{\otimes n}$, such that $\gamma_{n} \circ\left[\mathbf{F}_{C K}(T)\right] \circ \gamma_{m}^{-1}=\mathscr{F}_{T L}(T)$ for any flat $(m, n)$-tangle $T$.

Let us briefly recall from [CK14] how $\gamma_{n}$ is constructed. The group $K_{0}\left(A^{n}\right)$ is generated freely by indecomposable projectives $P_{a}:=e_{a} A^{n}$, one projective for each generalized cup diagram $a \in \mathscr{G} \mathcal{M}^{n}$. On the other hand, cup diagrams are flat tangle. The isomorphism $\gamma_{n}$ is defined by $\gamma_{n}\left(\left[P_{a}\right]\right):=\mathscr{F}_{T L}(a)\left(v_{-}^{\otimes \ell} \otimes v_{+}^{\otimes r}\right)$, where $\ell$ and $r$ count points of $a$ on the left and right platform respectively. 


\section{Quantization of the annular link homology}

This section discusses the construction and properties of the quantum annular link homology. We start with redefining the action of $\mathfrak{s l}_{2}$ in a way that motivates the search for the quantization. Section 6.2 contains a detailed construction of the quantum annular TQFT, which is then applied to (a quantization of) the formal bracket to obtain the new invariant.

\subsection{The action of $\mathfrak{s l}_{2}$ revisited}

The action of $\mathfrak{s l}_{2}$ on the annular link homology can be understood already at the level of the skein category. Consider now an operation that takes a flat tangle $T$ into the surface $\mathbb{S}^{1} \times T \subset \mathbb{A} \times I$. A closed loop in $T$ corresponds to a toroidal component of the surface, which is evaluated to 2 in $\mathscr{B} \mathcal{N}(\mathbb{A})$ due to the neck cutting relation. Hence, there is a well-defined functor $\mathbb{S}^{1} \times(-):\left.\mathscr{T} \mathscr{L}\right|_{q=1} \longrightarrow \mathscr{B} \mathcal{N}(\mathbb{A})$, where $\left.\mathscr{T} \mathscr{L}\right|_{q=1}$ is the Temperly-Lieb category specialized at $q=1$, see Appendix A.1. In fact, it takes values in $\mathscr{B} \mathscr{N}(\mathbb{A})$, the quotient of $\mathscr{B} \mathcal{N}(\mathbb{A})$ by the Boerner's relation that forces a dot to annihilate a connected surface with an essential circle in its boundary.

Remark. Because $\mathscr{B} \mathscr{N}(\mathbb{A})$ is both graded and additive, from now on we make two modification to $\mathscr{T L}$ : we introduce a formal degree shift despite all morphisms in $\mathscr{T} \mathscr{L}$ having degree 0 , and formal direct sums, so that $\mathscr{T} \mathscr{L}$ becomes a graded additive category. The functor $\mathscr{F}_{T L}$ extends naturally to a faithful functor $\mathscr{F}_{T L}: \mathscr{T L} \longrightarrow \mathrm{g} \mathscr{R e p}\left(\mathscr{U}_{q}\left(\mathfrak{s l}_{2}\right)\right)$ and $\mathbb{S}^{1} \times(-)$ is still well-defined when $q=1$.

Proposition 6.1. The functor $\mathbb{S}^{1} \times(-):\left.\mathscr{T} \mathscr{L}\right|_{q=1} \longrightarrow \mathscr{B} \mathscr{B N}(\mathbb{A})$ is an equivalence of categories.

Proof. By the Delooping Lemma, each object in $\mathscr{B} \mathscr{N}(\mathbb{A})$ is isomorphic to a collection of essential curves with shifted degree, whereas the neck cutting relation implies that morphisms between such collections are generated by incompressible surfaces, i.e. annuli [AF07]. These are graded morphisms only when the degree shifts of the collection of curves at the bottom and top agree. Hence, the functor $\mathbb{S}^{1} \times(-)$ is full and essentially surjective. Faithfulness follows, because the annuli are linearly independent in $\mathscr{B} \mathscr{B} \mathcal{N}(\mathbb{A})$, see [Rus09].

Specializing $q=1$ makes $\mathscr{F}_{T L}$ valued in g $\mathscr{R} e p\left(\mathfrak{s l}_{2}\right)$. We check directly that the triangle of functors

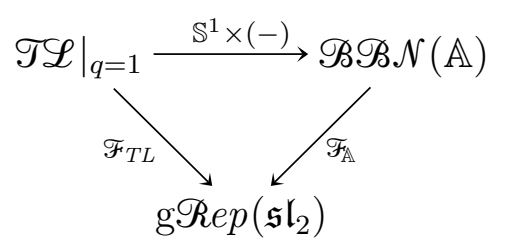

commutes. This equips the annular TQFT with an action of $\mathfrak{s l}_{2}$ that coincides with the one from [GLW15].

\subsection{Deformation of the annular skein category}

Our goal is to quantize the annular skein category, so that Proposition 6.1 holds for all values of $q$. We achieve this by identifying $\mathscr{B} \mathcal{N}(\mathbb{A})$ with the additive closure of the horizontal trace of the bicategory $\mathbf{B N}$ (taking the additive closure is necessary, because $h \operatorname{Tr}(\mathbf{B N})$ 
is only linear), then deforming the trace relation. Because the horizontal traces are compared with Bar-Natan skein categories, it is understood that they are made graded by introducing formal degree shifts.

Proposition 6.2. There is an equivalences of categories $\mathrm{hTr}^{\oplus}(\mathbf{B N}) \simeq \mathscr{B} \mathcal{N}(\mathbb{A})$.

Proof. The proof follows the same argument as the one of Theorem A.

The bicategory $\mathbf{B N}$ is locally pregraded and each cobordism $S$ is a homogeneous morphism of degree

$$
\operatorname{deg} S=\chi(S)-\frac{\# B}{4}-2 d
$$

where $B$ is the set of corners of $S$ and $d$ the number of dots. Thence, we can deform $\mathscr{B} \mathcal{N}(\mathbb{A})$ by taking quantum horizontal traces. Namely, we define the quantum Bar-Natan skein category of the annulus as the additive closure of the quantum horizontal trace

$$
\mathscr{B} \mathcal{N}_{q}(\mathbb{A}):=\operatorname{hTr}_{q}^{\oplus}(\mathbf{B N}) .
$$

This category admits the following graphical description. The identified boundaries of $(\mathbb{R} \times I) \times I$ form a membrane in the resulting solid torus, and the orientation of the core of $\mathbb{A}$ equips the membrane with a coorientation. Isotopic cobordisms are identified whenever the isotopy fixes the membrane. Otherwise, we scale the target cobordism according to the following rules:
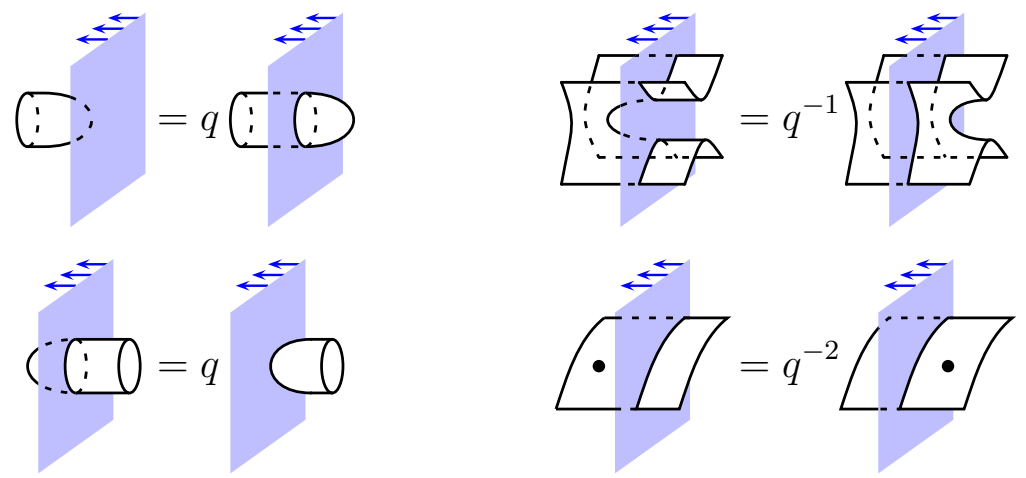

where, in each equality, the tangles draw on the membrane by the cobordism have the same formal shift. For instance, a torus wrapped once around the annulus evaluates to $q+q^{-1}$ :

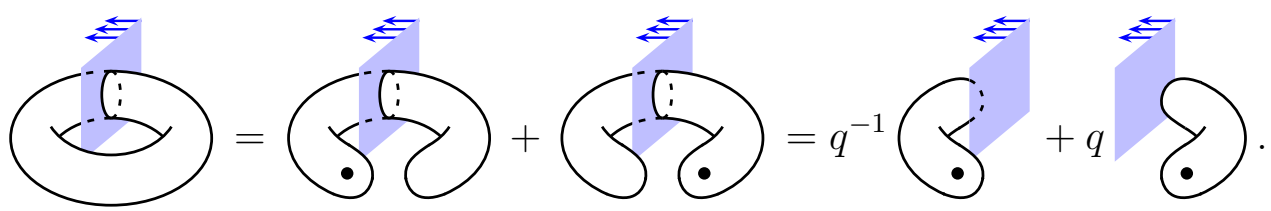

It follows also that $\left(1-q^{2}\right)$ annihilates a surface $S$ that has a connected component with both an essential boundary and a dot. Indeed, moving the dot along the essential boundary curve does not change the isotopy class of $S$, yet it requires to pass the dot through a membrane. Therefore, from now on we impose the Boerner relation, writing

$$
\mathscr{B} \mathscr{B} \mathcal{N}_{q}(\mathbb{A}):=\mathscr{B} \mathcal{N}_{q}(\mathbb{A}) / B=\mathrm{hTr}_{q}^{\oplus}(\mathbf{B N}) / B
$$

for the quotient category. According to (6.5), the Cartesian product with a circle is a well-defined functor

$$
\mathbb{S}^{1} \times(-): \mathscr{T} \mathscr{L} \longrightarrow \mathscr{B} \mathscr{B} \mathcal{N}_{q}(\mathbb{A})
$$

for any value of $q$. The rest of this section is devoted to prove the following statement. 
Theorem 6.3. There is a commuting diagram

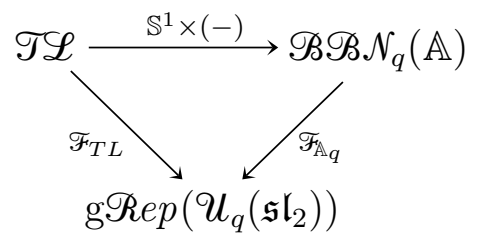

with the horizontal functor an equivalence of categories.

We first show the surjectivity of $\mathbb{S}^{1} \times(-)$. Hereafter we write $\mu=\{1\} \times \mathbb{R} \subset \mathbb{S}^{1} \times \mathbb{R}$ for the seam, the arc formed by identifying the boundaries of $\mathbb{R} \times I$.

Lemma 6.4. The canonical embedding $\mathfrak{i}: \operatorname{vTr}_{q}^{\oplus}(\mathbf{B N}) \longrightarrow \mathrm{h}_{q}^{\oplus}(\mathbf{B N})$ is an equivalence of categories.

Proof. The functor $\mathfrak{i}$ is full and faithful, see Section 3.4. Therefore, we need only to show that every object of $h \operatorname{Tr}_{q}^{\oplus}(\mathbf{B N})=\mathscr{B} \mathcal{N}_{q}(\mathbb{A})$ is isomorphic to one from the image of $\mathfrak{i}$.

Let $\Gamma$ be a collection of curves in $\mathbb{A}$. If $\alpha \subset \Gamma$ is an embedded arc such that $\partial \alpha=\mu \cap \alpha$, then we shall say that $\Gamma$ is retractible if there is an embedded disk $D \subset \mathbb{A}$ with interior disjoint from $\Gamma$, and which boundary is formed by $\alpha$ and the subarc of $\mu$ that lies between the two endpoints of $\alpha$, see Figure 11.

Assume first that $\Gamma$ has no retractible arcs, so that it is a collection of essential and trivial circles. Essential circles are in the image of $\mathfrak{i}$, and the trivial ones can be removed using the delooping isomorphism from Proposition 5.3.

It remains to show that collections of curves with no retractible arcs generate $\mathscr{B} \mathcal{N}_{q}(\mathbb{A})$. For that pick a retractible arc $\alpha$ in $\Gamma$ with a corresponding disk $D$ and apply an isotopy that pushes $\alpha$ across $D$, taking it off $\mu$. This procedure reduces the geometric intersection number of $\Gamma$ with $\mu$ by 2 . Hence, applying this step several times, we end up with $\Gamma^{\prime}$ that contains no retractible arcs, hence from the image of $i$.

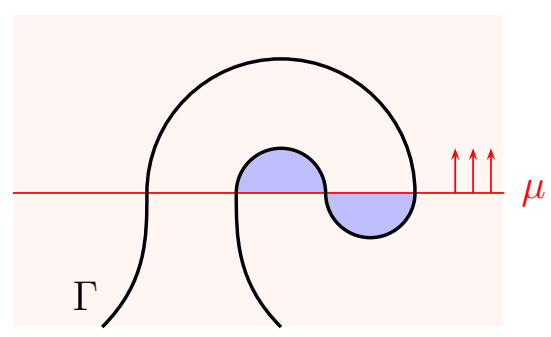

Figure 11: An example of a positive (to the left) and a negative (to the right) retractible arc, each with the retracting disk. The top arc is not retractible, but it will be after the positive arc is retracted.

Corollary 6.5. The functor $\mathbb{S}^{1} \times(-): \mathscr{T} \mathscr{L} \longrightarrow \mathscr{B} \mathscr{N _ { q }}(\mathbb{A})$ is full and essentially surjective.

Proof. In the view of (6.6) and Lemma 6.4 we only need to show that every cobordism in $\mathscr{B} \mathscr{B} \mathcal{N}_{q}(\mathbb{A})$ is a linear combination of those of the form $\mathbb{S}^{1} \times T$, where $T$ is a Temperly-Lieb diagram. We achieve that by using the Bar-Natan and the trace relations.

Using the neck-cutting relation we reduce first a surface $S$ to a linear sum of surfaces $S_{i}$ of genus 0. Because all closed components evaluate to scalars, we may assume each $S_{i}$ has a boundary component, which is an essential curve in $\mathbb{A}$ intersecting the seam 
once. Hence, $S_{i}$ is an annulus that intersects the membrane in an arc and, perhaps, in a collection of circles. The latter can be removed at the cost of some power of $q$ with the left relations in (6.4). The resulting surface is isotopic to $\mathbb{S}^{1} \times T$, where $T$ is the intersection of $S_{i}$ with the membrane. Furthermore, the Boerner relation prohibits the surface from carrying dots.

To show faithfulness of $\mathbb{S}^{1} \times(-)$, so that $\mathscr{B} \mathscr{B} \mathcal{N}_{q}(\mathbb{A})$ does not collapse, we construct the functor $\mathscr{F}_{\mathbb{A}_{q}}: \mathscr{B} \mathscr{B} \mathcal{N}_{q}(\mathbb{A}) \longrightarrow \operatorname{Mlod}(\mathbb{k})$ using the diagram

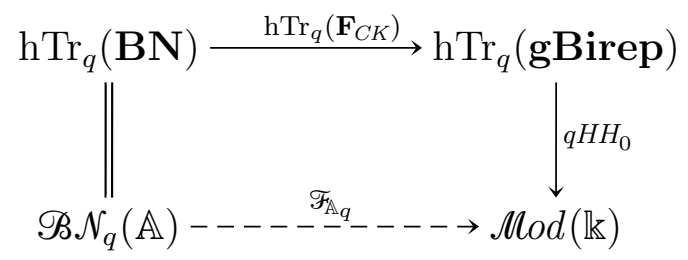

where $\operatorname{hr}_{q}\left(\mathbf{F}_{C K}\right)$ is the functor induced by the Chen-Khovanov TQFT. Here gBirep is the bimodule bicategory restricted to sweet bimodules, i.e. those that are finitely generated and projective as both left and right modules. This bicategory has duals, so that the shadow $q H H_{0}$ factorizes through the horizontal trace by Theorem 3.5, providing the right vertical map. Explicitly,

$$
\mathscr{F}_{\mathbb{A}_{q}}(\widehat{T}):=q H H_{0}\left(A^{n}, \mathbf{F}_{C K}(T)\right)
$$

for a flat $(n, n)$-tangle $T$. Equivalently, $\mathscr{F}_{\mathbb{A}_{q}}$ can be constructed by pulling back $q H H_{0}$ to $\mathbf{B N}$ along $\mathbf{F}_{C K}$ and factorizing it through the horizontal trace. The following justifies taking only the 0th Hochschild homology.

Proposition 6.6. Suppose $\mathbb{k}$ is flat over $\mathbb{Z}\left[q^{ \pm 1}\right]$. Then the inclusion $A_{0}^{n} \subset A^{n}$ induces an isomorphism of quantum Hochschild homology. In particular, the Chern character $h: K_{0}\left(A^{n}\right) \otimes_{\mathbb{Z}\left[q^{ \pm 1}\right]} \mathbb{k} \longrightarrow q H H_{0}\left(A^{n}\right)$ is an isomorphism and $q H H_{i}\left(A^{n}\right)=0$ for $i>0$.

Proof. It is enough to check the case $\mathbb{k}=\mathbb{Z}\left[q^{ \pm 1}\right]$. Consider quantum Hochschild homology as abelian groups and let $R$ be any ring. The Universal Coefficient Theorem provides a commuting diagram

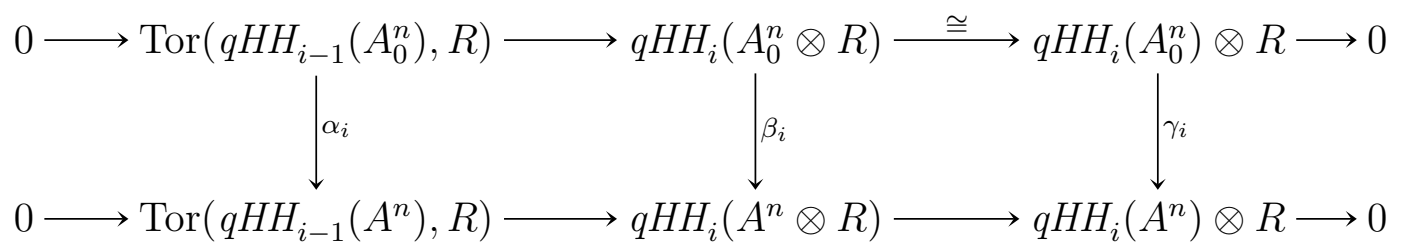

with exact rows, where the vertical homomorphisms are induced by the inclusion of algebras. Theorem 4.10 implies that $\beta_{i}$ is an isomorphism if $R=\mathbb{Z}_{p}$ for prime $p$, because the global dimension of $A^{n} \otimes \mathbb{Z}_{p}$ is finite [BS11] and simple modules are one-dimensional. Thus $\gamma_{0}$ is an isomorphism, because the left groups vanish when $i=0$. Hence, it is an isomorphism for $R=\mathbb{Z}$, and so must be $\alpha_{1}$. Using 5-Lemma we can now prove by induction that $\beta_{i}$ is an isomorphism for $R=\mathbb{Z}$ and any $i$, which shows the first claim.

The second claim follows from a direct computation. The algebra $A_{0}^{n} \cong \mathbb{k}^{2^{n}}$ is generated by $2^{n}$ orthogonal idempotents, so that $q H H_{0}\left(A_{0}^{n}\right) \cong \mathbb{k}^{2^{n}} \cong K_{0}\left(A^{n}\right) \otimes_{\mathbb{Z}\left[q^{ \pm 1}\right]} \mathbb{k}$ and higher homology vanishes.

Corollary 6.7. We have $q H H_{i}\left(A^{n}, \mathbf{F}_{C K}(T)\right)=0$ for a flat $(n, n)$-tangle $T$ and $i>0$. 
Proof. In the view of Lemma 6.4 every flat $(n, n)$-tangle $T$ is isomorphic in $\mathscr{B} \mathcal{N}_{q}(\mathbb{A})$ to the disjoint union of the identity tangle on $m$ points and $\ell$ trivial loops for some $m, \ell \geqslant 0$. Hence, $q H H_{i}\left(A^{n}, \mathbf{F}_{C K}(T)\right) \cong q H H_{i}\left(A^{m}\right) \otimes W^{\otimes \ell}$, where $W=\operatorname{span}_{\mathbb{k}}\left\{w_{+}, w_{-}\right\}$is the module assigned by $\mathbf{F}_{C K}$ to a circle. The thesis follows from Proposition 6.6.

We are now ready to show the main result of this section.

Proof of Theorem 6.3. The naturality of the Chern character provides a commuting square

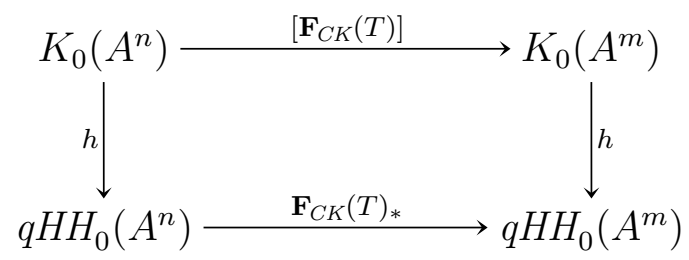

in which both compositions take the class $[P]$ of a projective to the quantum HattoringStallings trace of the identity morphism on $P \otimes_{A^{n}} \mathbf{F}_{C K}(T)$, see 3.8.2. The vertical arrows are isomorphisms by Proposition 6.6 and the top horizontal map can be identified with $\mathscr{F}_{T L}(T)$ due to Theorem 5.8. Finally, the bottom horizontal map coincides with $\mathscr{F}_{\mathbb{A}_{q}}$, because it is the result of applying the pullback shadow $\left(\mathbf{F}_{C K}\right)^{*} q H H_{0}$ to the morphism in $\mathrm{vTr}_{q}(\mathbf{B N}) \subset \mathrm{h} \operatorname{Tr}_{q}(\mathbf{B N})$ represented by the square $[T, I \times T]$.

\subsection{Quantum annular homology}

We use the deformed skein category together with the functor $\mathscr{F}_{\mathbb{A}_{q}}$ to produce a quantization of the annular link homology. The construction follows the usual pattern.

1) Choose an annular link diagram $D$ that is transverse to the seam $\mu \subset \mathbb{A}$. In particular, all crossings are assumed to be away from $\mu$.

2) Construct the formal bracket $\llbracket D \rrbracket_{q}$ in $\operatorname{Com}_{/ h}^{b}\left(\mathscr{B} \mathscr{B} \mathcal{N}_{q}(\mathbb{A})\right)$ as explained in Section 5.1, where we use the subscript $q$ to emphasize that the resolutions of $D$ are taken in the quantized skein category

3) Apply the functor $\mathscr{F}_{\mathbb{A}_{q}}$ component-wise to $\llbracket D \rrbracket_{q}$ to get the quantum annular chain complex $C K h_{\mathbb{A}_{q}}(D):=\mathscr{F}_{\mathbb{A}_{q}} \llbracket D \rrbracket_{q}$.

The quantum annular homology $K h_{\mathbb{A}_{q}}(L)$ of an annular link $L$ is defined as the homology of $C K h_{\mathbb{A}_{q}}(D)$. It is a triply graded $\mathbb{k}$-module with a homological grading, a quantum grading coming from the grading on $\mathbf{F}_{C K}$, and an annular grading arising from the $\mathfrak{s l}_{2}$ weight decomposition. In what follows we prove that it is well-defined and examine functoriality with respect to annular link cobordisms. We begin with a detailed look on the quantized formal bracket. In what follows, we say that a functor or a shadow is projective or q-projective if it is defined on 2-morphisms up to scaling by \pm 1 or $\pm q^{ \pm k}$ for some $k \in \mathbb{Z}$ respectively.

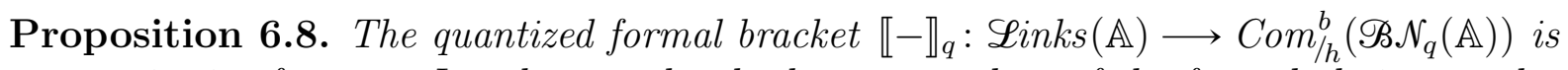
a q-projective functor. In other words, the homotopy class of the formal chain complex $\llbracket D \rrbracket_{q}$ is an invariant of annular links and an annular link cobordism $W: L \longrightarrow L^{\prime}$ induces a formal chain map $\llbracket W \rrbracket_{q}: \llbracket D \rrbracket_{q} \longrightarrow \llbracket D^{\prime} \rrbracket_{q}$, defined up to an overall sign and power of $q$, where $D$ and $D^{\prime}$ are diagrams of $L$ and $L^{\prime}$ respectively.

Proof. Let $\mathbb{k}$ Tan be the linear extension of the tangle bicategory, 2-morphisms of which are finite sums of tangle cobordisms with coefficients in $\mathbb{k}$. Motivated by Theorem A we define $\operatorname{Links}_{q}(\mathbb{A}):=h \operatorname{Tr}_{q}(\mathbb{k}$ Tan $)$. It is a deformed linear extension of the category 
of annular links, in which an annular link cobordism gets scaled by a power of $q$ when isotoped through the membrane. Clearly, the formal bracket on Tan extends to $\mathbb{k}$ Tan.

The universal shadow $\widehat{(-)}: \mathbf{B N} \longrightarrow \mathscr{B}_{\mathcal{N}}(\mathbb{A})$, defined as the annular closure of flat tangles, induces a Lefschetz shadow on $\operatorname{Com}_{h}^{b}(\mathbf{B N})$, see Lemma 3.16. Its pullback to $\mathbb{k}$ Tan along $\llbracket-\rrbracket$ is thence a projective shadow. The universality of the horizontal trace gives then a projective functor $\llbracket-\rrbracket_{q}^{\prime}: \mathscr{L i n k s}_{q}(\mathbb{A}) \longrightarrow \operatorname{Com}_{/ h}^{b}\left(\mathscr{B} \mathcal{N}_{q}(\mathbb{A})\right)$. One can easily check that $\llbracket W \rrbracket_{q}^{\prime}=\llbracket W \rrbracket_{q}$ for a fixed presentation of a link cobordism $W$, and it remains to check how dropping the membrane affects the bracket. For that choose isotopic link cobordisms $W$ and $W^{\prime}$ that are related by a trace move. Regarded as morphisms in $\mathscr{L} i n k s_{q}$, they satisfy a relation $W=q^{k} W^{\prime}$ for some $k$. Hence, $\llbracket W \rrbracket_{q}=\llbracket W \rrbracket_{q}^{\prime}= \pm q^{k} \llbracket W^{\prime} \rrbracket_{q}^{\prime}= \pm q^{k} \llbracket W^{\prime} \rrbracket_{q}$, which ends the proof.

Theorem B. The quantum annular homology $K h_{\mathbb{A}_{q}}(L)$ is a triply graded invariant of an annular link L, which is q-projectively functorial with respect to annular link cobordisms. Moreover, it admits an action of the quantum group $\mathcal{U}_{q}\left(\mathfrak{s l}_{2}\right)$ that commutes with the differential and the maps induced by annular link cobordisms intertwine this action.

Proof. It follows from Proposition 6.8 that the functor $C K h_{\mathbb{A}_{q}}(-):=\mathscr{F}_{\mathbb{A}_{q}} \llbracket-\rrbracket_{q}$ is $q-$ projective functor and the action of $\mathcal{U}_{q}\left(\mathfrak{s l}_{2}\right)$ follows from Theorem 6.3.

It was conjectured in [AGW15] that the Hochschild homology of Chen-Khovanov complexes recovers the annular chain complexes, with a proof for the algebras $A^{1, n-1}$, the next-to-highest weight subalgebra of $A^{n}$. The conjecture follows from Theorem 6.3 and the computation of Hochschild homology in Corollary 6.7.

Theorem C. Let $\widehat{T}$ be the annular closure of an $(n, n)$-tangle $T$. Then there is an isomorphism

$$
K h_{\mathbb{A}_{q}}(\widehat{T}) \cong q H H_{\bullet}\left(A^{n}, C_{C K}(T)\right),
$$

natural with respect to chain maps associated to tangle cobordisms. The annular grading in $K h_{\mathbb{A}}(\widehat{T})$ corresponds to the weight decomposition of $C_{C K}(T)$.

Proof. The right hand side of (6.12) is the total homology of the bicomplex

$$
\operatorname{coInv}_{q}\left(C_{C K}^{\bullet}(T) \underset{A^{n}}{\otimes} R_{\bullet}\left(A^{n}\right)\right),
$$

where $R_{\bullet}\left(A^{n}\right)$ is the bar resolution of $A^{n}$. Consider the associated spectral sequence $E^{r}$ that starts with the Hochschild differential. The first page $E_{i j}^{1}=q H H_{j}\left(A^{n}, C_{C K}^{i}(T)\right)$ has vanishing rows except the one with $j=0$ due to Corollary 6.7. Hence, the sequence collapses at the second page with $E_{i 0}^{2}=K h_{\mathbb{A}_{q}}^{i}(\widehat{T})$ and $E_{i j}^{2}=0$ for $r \neq 0$, which ends the proof.

There is another spectral sequence $\left\{{ }^{\prime} E^{r}\right\}$ associated to $\operatorname{coInv}_{q}\left(C_{C K}^{\bullet}(T) \otimes_{A^{n}} R \bullet\left(A^{n}\right)\right)$, where the Chen-Khovanov differential is computed first. Its second page

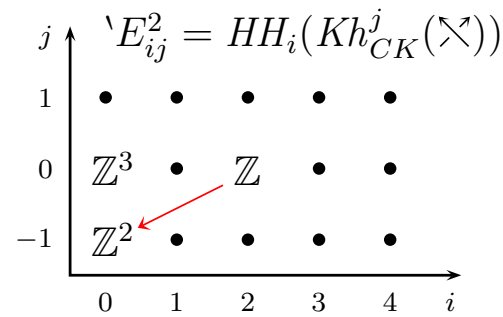

$$
{ }^{\prime} E_{i j}^{r}=q H H_{i}\left(A^{n}, K h_{C K}^{j}(T)\right)
$$

is already an invariant of the annular link $\widehat{T}$. Contrary to the previous case, this sequence may not collapse immediately. The second page for $T=\lambda$ has three non-trivial entries and there is a non-trivial differential that kills two generators, see the diagram to the left for the case $\mathbb{k}=\mathbb{Z}$ and $q=1$. It is the third page that agrees with the annular homology of $\widehat{T}$, which is the homology of $\left(W \longrightarrow V^{\otimes 2}\right)$ with $W$ in homological degree -1 . 


\subsection{Homology for $(2, n)$ torus links}

Consider the subcategory $\mathscr{E}_{2} \subset \mathscr{B} \mathscr{B} \mathcal{N}_{q}(\mathbb{A})$ generated by objects intersecting the seam $\mu$ in exactly two points. Note that every such object is of the form $\Gamma_{I} \cup \Gamma_{N}$, where $\Gamma_{N}$ is a (possibly empty) union of trivial circles not intersecting $\mu$, and $\Gamma_{I}$ is either a trivial circle intersecting $\mu$ in two points, or a pair of essential circles, each intersecting $\mu$ in a single point.

In what follows we shall write $W$ or $W_{I}$ for the module assigned to a trivial circle depending on whether it is disjoint from $\mu$ or not. $W$ is freely generated by $w_{+}$and $w_{-}$, the images of $1 \in \mathbb{k}$ under the maps induced by a cup cobordism disjoint from the membrane $\boldsymbol{U}=\mu \times I$, without and with a dot respectively. To pick generators for $W_{I}$ consider a cup cobordism that intersects $\boldsymbol{M}$ in a single arc $\alpha$ and define $w_{+}, w_{-}^{-}$and $w_{-}^{+}$ as the images of $1 \in \mathbb{k}$ under the maps induced by the cobordism respectively without any dot, a dot on the negative side of $\boldsymbol{M}$, and a dot on the positive side of $\boldsymbol{M}$. All three generate $W_{I}$, but they are not linearly independent: $w_{-}^{-}=q^{2} w_{-}^{+}$.

We can represent elements of the modules graphically as in Section 5.3: the generators of $V$ are visualized by orienting the essential circles, and those of $W$ and $W_{I}$ are given as the trivial circle without or with a dot. The relation in $W_{I}$ between $w_{-}^{-}$and $w_{-}^{+}$can be then written diagrammatically as

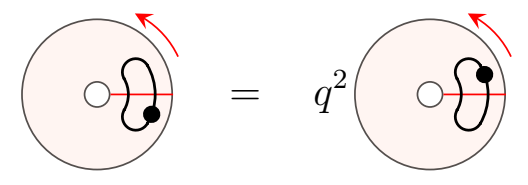

Here we choose the counter-clockwise orientation of the core of the annulus, so that the seam $\mu$ is cooriented upwards. Because the intertwners (A.6) are not symmetric, the essential circles must be ordered. We choose the left-to-right ordering read from the seam.

Capping off a trivial circle touching the seam vanishes on $w_{+}$and takes $w_{-}^{ \pm}$to $q^{\mp 1}$, as the result is a sphere without or with a dot respectively, but intersecting the membrane, and isotoping it off the membrane using (6.4) introduces a power of $q$. It is now straightforward to compute the saddle cobordisms in $\mathscr{E}_{2}$ using the comparison with $\mathscr{F}_{T L}$. A merge of two essential circles followed by capping off the trivial circle is the evaluation map, which implies the following surgery rules :
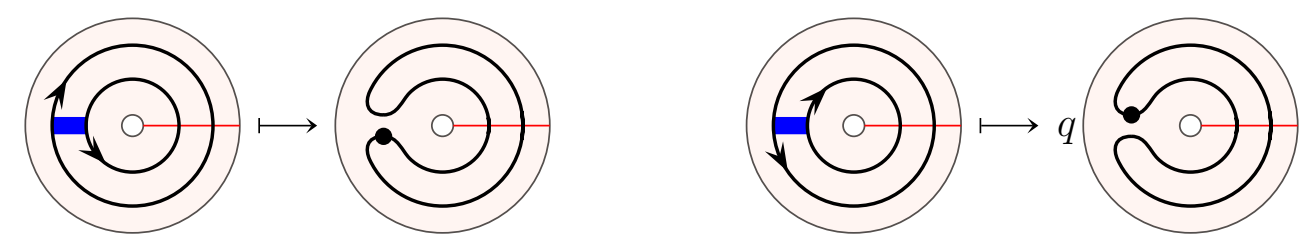

The other saddle cobordism is even easier to find out. For degree reasons it must vanish if the trivial circle carries a dot, and otherwise it is the coevaluation map:

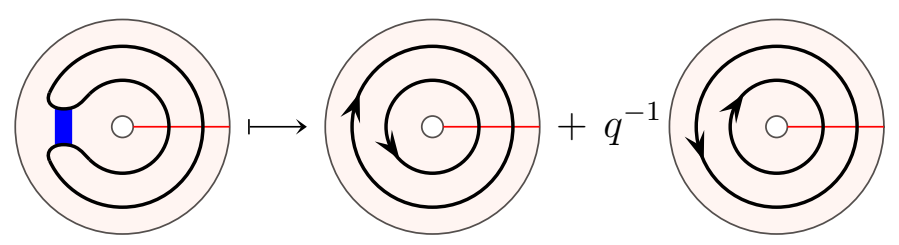

Fix $n>0$ and let $T_{2, n}$ denote the annular $(2, n)$ torus link: the annular closure of the braid $\sigma^{-n}$, where $\sigma$ is the positive generator of the 2 -strand braid group. 
Proposition 6.9. The quantum annular Khovanov homology of the annular $(2, n)$ torus link is given by

$$
K h_{\mathbb{A}_{q}}^{i, j}\left(T_{2, n}\right)= \begin{cases}V_{2} & \text { if } i=0 \text { and } j=-n, \\ V_{0} /\left(q^{2}+(-1)^{i}\right) & \text { if }-n+1 \leqslant i \leqslant-1 \text { and } j=2 i-n, \\ K\left(q^{2}-(-1)^{i}\right) & \text { if }-n \leqslant i \leqslant-2 \text { and } j=2 i-n+2, \\ V_{0} & \text { if } i=-n \text { and } j=-3 n, \\ 0 & \text { else, }\end{cases}
$$

where $V_{2}:=(V \otimes V) / \operatorname{span}_{\mathbb{k}}\left\{v_{+} \otimes v_{-}+q^{-1} v_{-} \otimes v_{+}\right\}$is the simple representation of $\boldsymbol{U}_{q}\left(\mathfrak{s l}_{2}\right)$ of dimension 3, and $K(a):=\left\{v \in V_{0} \mid a v=0\right\}$ for any $a \in \mathbb{k}$.

Here $i$ and $j$ represent respectively the homological and the quantum degree as defined in (5.14). The latter is denoted by $j^{\prime}$ in [GLW15].

Proof. Let $D \subset \mathbb{A}$ be a standard diagram for $T_{2, n}$ such that cutting $D$ along $\mu$ results in a diagram for $\sigma^{-n}$. Then each resolution of $D$ belongs to $\mathscr{E}_{2}$. We introduce the notations $u_{q}, l_{q}: W_{I} \longrightarrow W_{I}$ for the maps that put a dot on the positive and negative side of the circle respectively, and $w_{q}: W_{I} \longrightarrow V \otimes V$ for the split map. Explicitly,

$$
\begin{aligned}
u_{q}\left(w_{+}\right) & =w_{-}^{+}, & u_{q}\left(w_{-}\right) & =0, \\
l_{q}\left(w_{+}\right) & =w_{-}^{-}=q^{2} w_{-}^{+}, & l_{q}\left(w_{-}\right) & =0, \\
w_{q}\left(w_{+}\right) & =v_{+} \otimes v_{-}+q^{-1} v_{-} \otimes v_{+}, & w_{q}\left(w_{-}^{ \pm}\right) & =0 .
\end{aligned}
$$

Let $\{m\}$ denote the grading shift functor which raises the $j$-degree by $m$. Arguing as in [Kh99, Proposition 26], one can show that $C K h_{\mathbb{A}_{q}}(D)$ is quasi-isomorphic to the chain complex

$$
\begin{aligned}
& 0 \longrightarrow W_{I}\{-3 n+1\} \stackrel{\partial^{-n}}{\longrightarrow} W_{I}\{-3 n+3\} \stackrel{\partial^{-n+1}}{\longrightarrow} \ldots \\
& \stackrel{\partial^{-3}}{\longrightarrow} W_{I}\{-n-3\} \stackrel{\partial^{-2}}{\longrightarrow} W_{I}\{-n-1\} \stackrel{\partial^{-1}}{\longrightarrow} V_{1} \otimes V_{1}^{*}\{-n\} \longrightarrow 0
\end{aligned}
$$

where $\partial^{-1}=w_{q}$ and $\partial^{i}=u_{q}-(-1)^{i} l_{q}$ for $-n \leq i \leq-2$. One can write the above complex more explicitly by writing each $W_{I}$ as a direct sum

$$
W_{I}=\operatorname{span}_{\mathbb{k}}\left\{w_{+}, w_{-}\right\}=V_{0}\{+1\} \oplus V_{0}\{-1\},
$$

and by noting that the map $u_{q}-(-1)^{i} l_{q}$ is given by

$$
u_{q}-(-1)^{i} l_{q}=\left(\begin{array}{cc}
0 & 0 \\
0 & 1-(-1)^{i} q^{2}
\end{array}\right)
$$

with respect to this direct sum decomposition. It then follows that the above complex is isomorphic to a direct sum of complexes

$$
\begin{aligned}
& \left(0 \longrightarrow V_{0} \stackrel{w_{q}}{\longrightarrow} V_{1} \otimes V_{1} \longrightarrow 0\right)\{-n\} \\
& \left(0 \longrightarrow V_{0} \stackrel{1+(-1)^{i} q^{2}}{\longrightarrow} V_{0} \longrightarrow 0\right)\{2 i-n\} \quad \text { for }-n+1 \leq i \leq-1 \\
& \left(0 \longrightarrow V_{0} \longrightarrow 0\right)\{-3 n\}
\end{aligned}
$$

where, in each of these complexes, the bidegree of the rightmost nonzero term is supported on the diagonal $j=2 i-n$. The proposition now follows by passing to homology. 


\begin{tabular}{||c||c|c|c|c|c|c||}
\hline \hline$j$ & -5 & -4 & -3 & -2 & -1 & 0 \\
\hline \hline-5 & & & & & & $V_{2}$ \\
\hline-7 & & & & $V_{0}^{\dagger}$ & $V_{0}^{\dagger}$ & \\
\hline-9 & & & $V_{0}^{\ddagger}$ & $V_{0}^{\ddagger}$ & & \\
\hline-11 & & $V_{0}^{\dagger}$ & $V_{0}^{\dagger}$ & & & \\
\hline-13 & $V_{0}^{\ddagger}$ & $V_{0}^{\ddagger}$ & & & & \\
\hline-15 & $V_{0}$ & & & & & \\
\hline \hline
\end{tabular}

Table 1: The quantum annular homology for the torus knot $T_{2,5}$. The representations marked by a dagger $(\dagger)$ only occur if $q^{2}=1$, and the ones marked by a double dagger $(\ddagger)$ only occur if $q^{2}=-1$. The unmarked representations are always there.

If $\mathbb{k}$ is a field and $q^{2} \neq \pm 1$, then $q^{2}+(-1)^{i}$ is invertible. It follows from the above proof that in such a case

$$
K h_{\mathbb{A}_{q}}^{i, j}(D)= \begin{cases}V_{2} & \text { if }(i, j)=(0,-n), \\ V_{0} & \text { if }(i, j)=(-n,-3 n), \\ 0 & \text { else. }\end{cases}
$$

On the other hand, the quantum homology contains additional copies of $V_{0}$ if $q^{2}= \pm 1$, see Table 1. This illustrates that quantum annular homology is in general richer than the non-quantized theory, which corresponds to the case $q=1$.

\section{Applications and generalizations}

Here we discuss functorial properties of the quantum annular link homology, as well as generalizations, such as the quantized APS homology for links in a thickened Möbius or the twisted annular homology (which provides a way to describe homology of satellite knots).

\subsection{Invariants for annular link cobordisms}

It follows from Theorem B that the quantum annular homology assigns a chain map to annular link cobordisms. Let us now recall how this map is computed.

Let $L$ and $L^{\prime}$ by annular closures of an $(m, m)$-tangle $T$ and an $(n, n)$-tangle $T^{\prime}$ respectively. A link cobordism $W: L \longrightarrow L^{\prime}$ intersects the membrane in an $(n, m)$-tangle $P$, so that it can be represented by a tangle cobordism $\widetilde{W}: P T \longrightarrow T^{\prime} P$. Applying the Chen-Khovanov functor results in a square

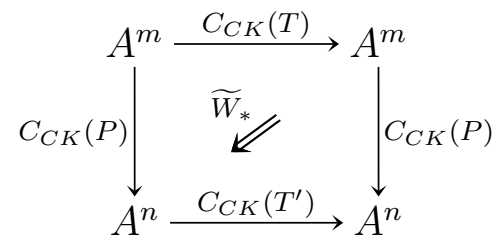

The quantum annular complex is computed by replacing each component of the ChenKhovanov complex with its Hochschild homology. This is an example of a Lefschetz 
shadow discussed in Section 3.6. Hence, the component of the chain map $W_{*}$ at homological degree $i$ and quantum degree $j$ is given by summing up the compositions

$$
\begin{aligned}
q H H_{0}\left(A^{m}, C_{C K}^{i, j}(T)\right) & \stackrel{\mathrm{coev}_{*}}{\longrightarrow} q H H_{0}\left(A^{m}, C_{C K}^{i, j}(T) \underset{A^{m}}{\otimes} C_{C K}^{i^{\prime}, j}(P) \underset{A^{n}}{\otimes} C_{C K}^{i^{\prime}, j}(P)^{*}\right) \\
& \stackrel{\widetilde{W}_{*}}{\longrightarrow} q H H_{0}\left(A^{m}, C_{C K}^{i^{\prime}, j}(P) \underset{A^{n}}{\otimes} C_{C K}^{i, j}\left(T^{\prime}\right) \underset{A^{n}}{\otimes} C_{C K}^{i^{\prime}, j}(P)^{*}\right) \\
& \stackrel{(-1)^{i^{\prime}\left(i+i^{\prime}\right)} \theta}{\longrightarrow} q H H_{0}\left(A^{n}, C_{C K}^{i, j}\left(T^{\prime}\right) \underset{A^{n}}{\otimes} C_{C K}^{i^{\prime}, j}(P)^{*} \underset{A^{m}}{\otimes} C_{C K}^{i^{\prime}, j}(P)\right) \\
& \stackrel{e v_{*}}{\longrightarrow} q H H_{0}\left(A^{n}, C_{C K}^{i, j}\left(T^{\prime}\right)\right)
\end{aligned}
$$

over all indices $i^{\prime}$. Notice that the third map takes the class of $x \otimes y \otimes \alpha$ to the class of $(-1)^{i^{\prime}\left(i+i^{\prime}\right)} q^{\text {qdeg }(x)} y \otimes \alpha \otimes x$.

Proposition 7.1. Let the cobordism $W: L \longrightarrow L$ trace the rotation of $L$ along the annulus. The induced map $K h_{\mathbb{A}_{q}}(W)$ scales $x \in K h_{\mathbb{A}_{q}}(L)$ by $q^{\mathrm{qdeg}(x)}$.

Proof. Write $L$ as the annular closure of $T$. Then $W$ is represented by the identity cobordism from $T T$ to itself, and the only components (7.2) that contribute are those with $i=i^{\prime}$. In particular, the second map scales the argument by $q^{j}$, but does not change the sign.

It follows that quantum annular homology detects twists: the chain map $W_{*}$ in the above proposition is not scaled identity. Even more, if a resolution $T_{\zeta}$ of $T$ is a collection of $n$ vertical lines, the restriction of the twist to $T_{\zeta}$ is $q^{d}$, where $d$ is the degree shift of $T_{\zeta}$ in the complex. Indeed, the component of the twist is represented by

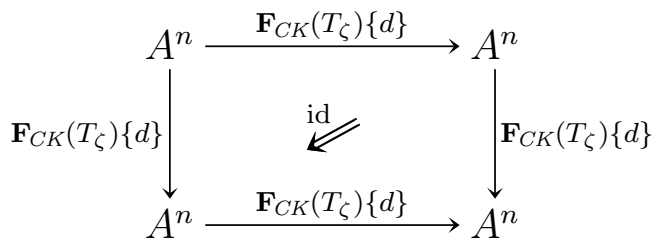

whereas the identity map has $\mathbf{F}_{C K}\left(T_{\zeta}\right)$ with no degree shifts as vertical maps.

Because $K h_{\mathbb{A}_{q}}$ is defined using a Lefschetz shadow, we can use Corollary 3.22 to compute the invariant for closed surfaces.

Theorem D. Let $\widehat{W} \subset \mathbb{S}^{1} \times \mathbb{R}^{3}$ be a closed surface obtained as an annular closure of a link cobordism $W: L \longrightarrow L$ with $L \subset \mathbb{R}^{3}$. Then $K h_{\mathbb{A}_{q}}(\widehat{W})=\Lambda\left(W_{*}\right)$ is the graded Lefschetz trace of $W_{*}: K h(L) \longrightarrow K h(L)$, the endomorphism of the Khovanov homology of $L$. In particular, $K h_{\mathbb{A}_{q}}\left(\mathbb{S}^{1} \times L\right)$ coincides with the Jones polynomial $J(L)$.

Proof. The first statement is an immediate consequence of Corollary 3.22. Applying it to $W=L \times I$ results in computing the graded Euler characteristic of $K h(L)$, the Khovanov homology of $L$.

The APS homology does not distinguish closed surfaces. Hence, $K h_{\mathbb{A}_{q}}$ is a nontrivial deformation of the annular $\mathfrak{s l}_{2}-$ homology. 


\subsection{An action of tangles on cablings}

The action of the braid group on the annular homology of a cabling of a framed annular knot $K$ was studied in [GLW15]. In what follows we compute the action of the entire category of oriented tangles on the quantum annular homology of cablings of $K$. We work in this section in characteristic 2, so that the Chen-Khovanov functor is strictly functorial.

To a framed knot $K \subset \mathbb{A} \times I$ we can associate an embedding $\nu_{K}: \mathbb{A} \times I \longrightarrow \mathbb{A} \times I$ with the tubular neighborhood as its image. It determines a functor $K_{*}: \operatorname{Tan} \longrightarrow \operatorname{Links}(\mathbb{A})$ such that

- a collection of oriented points $B \subset \mathbb{R}^{2}$ is taken to $K^{B}:=\nu_{K}\left(\mathbb{S}^{1} \times B\right)$, an oriented cabling of $K$, and

- an oriented tangle $T \subset \mathbb{R}^{2} \times I$ is mapped to the oriented cobordism $K^{T}:=\nu_{K}\left(\mathbb{S}^{1} \times T\right)$ between the cablings.

Applying the quantum annular homology produces a map of homology

$$
K h_{\mathbb{A}_{q}}\left(K^{T}\right): K h_{\mathbb{A}_{q}}\left(K^{B}\right) \longrightarrow K h_{\mathbb{A}_{q}}\left(K^{B^{\prime}}\right)
$$

for any oriented tangle $T \in \operatorname{Tan}\left(B, B^{\prime}\right)$, defined up to an overall power of $q$. These give a projectively functorial action of $\mathscr{T} a n$, i.e.

$$
K h_{\mathbb{A}_{q}}\left(K^{T^{\prime} T}\right)=q^{k}\left(K h_{\mathbb{A}_{q}}\left(K^{T^{\prime}}\right) \circ K h_{\mathbb{A}_{q}}\left(K^{T}\right)\right)
$$

for composable tangles $T, T^{\prime}$, and some $k \in \mathbb{Z}$.

This action has an interpretation in the framework of horizontal traces when $K$ is the annular closure of a framed $(1,1)$-tangle $\tau$. Denote by $\nu_{\tau}: \mathbb{R}^{2} \times I \longrightarrow \mathbb{R}^{2} \times I$ the associated parametrization of the tubular neighborhood of $\tau$ and choose a tangle $T$ with collections of points $B$ and $B^{\prime}$ as its bottom and top boundary respectively. Then the cobordism $K^{T}$ intersects the membrane in $T$ and is represented by the square

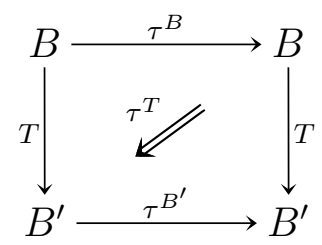

where $\tau^{B}:=\nu_{\tau}(B \times I)$ and $\tau\left(B^{\prime}\right):=\nu_{\tau}\left(B^{\prime} \times I\right)$ are cablings of $\tau$, and $\tau^{T}:=\mu_{\tau}(T \times I)$ traces the isotopy that slides $T$ along $\tau$. We use this to prove that the action satisfies the Jones relation.

Theorem E. Let $K$ be a framed annular link, considered as an object in $\mathscr{L}$ inks $s_{q}(\mathbb{A})$. There is a functorial action of Tan on the quantum annular homology of oriented cablings of $K$, that takes a tangle $T$ to the chain map $K h_{\mathbb{A}_{q}}\left(K^{T}\right)$, and which intertwines the action of $\mathcal{U}_{q}\left(\mathfrak{s l}_{2}\right)$. It factors through the Jones skein relation

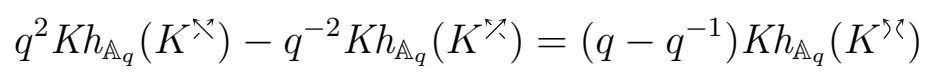

if $K$ intersects the membrane in one point.

Proof. Functoriality has been discussed before and compatibility with the action of $\boldsymbol{U}_{q}\left(\mathfrak{s l}_{2}\right)$ follows from the construction - the action of $\mathscr{T}$ an is already defined at the level of the quantized formal bracket. It remains to show that the Jones relation holds. We prove it for the formal bracket. 
Let $K$ be the annular closure of a $(1,1)$-tangle, represented by a tangle diagram $\tau$. Given a finite sequence $\underline{\epsilon}=\left(\epsilon_{1}, \ldots, \epsilon_{k}\right)$ with $\epsilon_{i} \in\{-1,+1\}$ we write $\tau^{\underline{\epsilon}}$ for the oriented $k$-cabling of $\tau$, in which each $i$-th cable is oriented parallel to $\tau$ when $\epsilon_{i}=+1$ and opposite to $\tau$ otherwise. We write $\tau^{k}$ when each $\epsilon_{i}=1$. Up to degree shifts, the formal bracket depends only on $k$. Explicitly,

$$
\llbracket \tau^{\underline{\epsilon}} \rrbracket=\llbracket \tau^{k} \rrbracket\left[\frac{w(\tau)}{2}\left(k^{2}-\|\underline{\epsilon}\|^{2}\right)\right]\left\{\frac{3 w(\tau)}{2}\left(k^{2}-\|\underline{\epsilon}\|^{2}\right)\right\},
$$

where we write $w(T)=n_{+}(T)-n_{-}(T)$ for the writhe of $T$ and $\|\underline{\epsilon}\|:=\sum \epsilon_{i}$. Notice that $\|\underline{\epsilon}\| \equiv k \bmod 2$, so that both numbers are integers. To derive this formula it is enough to compare degree shifts of a single resolution, which is left as an exercise.

It follows from functoriality of the action and (7.6) that the Jones relation has to be checked only for the first three tangles shown below:

$$
\begin{aligned}
& \left.\uparrow \cdots \bigwedge_{i} \cdots\right\rceil_{k+1} \\
& \left.\uparrow_{0} \cdots \bigwedge_{i}^{\wedge} \cdots\right|_{i+1} \\
& \uparrow_{0} \cdots \uparrow_{i} \uparrow_{i+1} \cdots \uparrow_{k} \\
& T_{0} \\
& \left.\uparrow \cdots \swarrow_{i}^{\smile} \cdots\right|_{k+1} \\
& T_{+}
\end{aligned}
$$

We use the forth one to express the formal brackets of $T_{+}$and $T_{-}$as mapping cones following Proposition 5.2, obtaining distinguished triangles

$$
\begin{aligned}
& \llbracket T_{0} \rrbracket[-1]\{1\} \stackrel{s d}{\longrightarrow} \llbracket T_{h} \rrbracket[-1]\{2\} \stackrel{\text { in }}{\longrightarrow} \llbracket T_{+} \rrbracket \stackrel{p r}{\longrightarrow} \llbracket \llbracket T_{0} \rrbracket\{1\} \\
& \llbracket T_{h} \rrbracket\{-2\} \stackrel{s d}{\longrightarrow} \llbracket \llbracket T_{0} \rrbracket\{-1\} \stackrel{i n}{\longrightarrow} \llbracket T_{-} \rrbracket \stackrel{p r}{\longrightarrow} \llbracket T_{h} \rrbracket[1]\{-2\}
\end{aligned}
$$

in which the left morphisms are saddle cobordisms in BN. Consider now the diagram

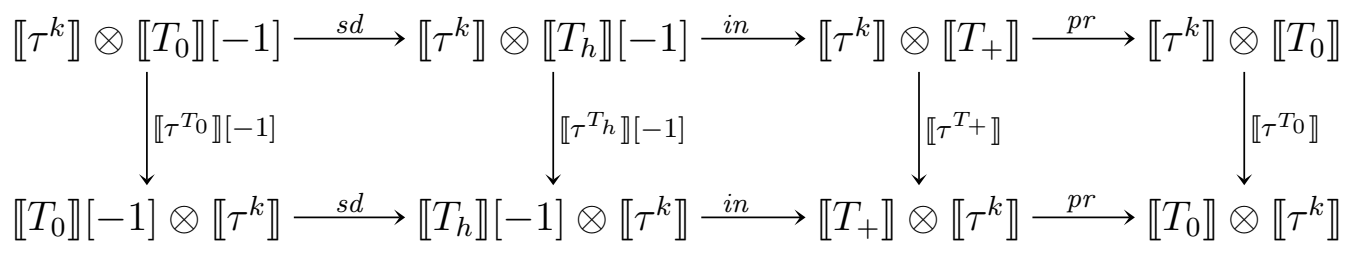

where the quantum degree shifts are dropped for clarity. The left and right vertical maps are identities, whereas those in the middle are induced by a sequence of Reidemeister moves. The left square commutes up to a formal chain homotopy $H$ by the functoriality of $\llbracket-\rrbracket$. In particular, $\llbracket \tau^{T_{0}} \rrbracket$ and $\llbracket \tau^{T_{h}} \rrbracket$ together with $H$ induce a chain map

$$
\alpha=\left(\begin{array}{cc}
\llbracket \tau^{T_{0}} \rrbracket & 0 \\
-H & \llbracket \tau^{T_{h}} \rrbracket[-1]
\end{array}\right): \llbracket \tau^{k} \rrbracket \otimes \operatorname{cone}(s d) \longrightarrow \operatorname{cone}(s d) \otimes \llbracket \tau^{k} \rrbracket
$$

that would make the other squares in (7.7) commute when placed as the third vertical map. Hence, it is enough to show that $\alpha$ and $\llbracket \tau^{T_{+}} \rrbracket$ are chain homotopic.

It is shown in [BN05, Section 8] that every degree 0 automorphism of $\llbracket T \rrbracket$ is homotopic to \pm id if $T$ is obtained from a crossingless tangle by twisting its endpoints. Therefore, there exists at most one homotopy equivalence $\llbracket T \rrbracket \simeq \llbracket T^{\prime} \rrbracket$ when $T$ and $T^{\prime}$ are two such tangles (as we work over $\mathbb{Z}_{2}$ ). In particular, there are unique homotopy equivalences

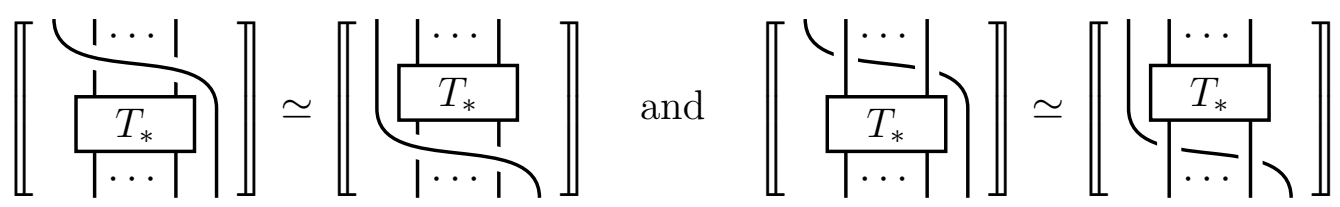


where $T_{*}$ is any of the four tangles $T_{+}, T_{-}, T_{0}$, or $T_{h}$. On the other hand, each map $\llbracket \tau^{T_{*}} \rrbracket$ is a composition of the above equivalences, and so is the chain map $\alpha$. Hence, $\alpha$ and $\llbracket \tau^{T_{+}} \rrbracket$ agree up to a chain homotopy, which makes the middle and right squares in $(7.7)$ commute in the homotopy category.

The quantum annular bracket is a quantum Lefschetz shadow on $\operatorname{Com}_{h}^{b}(\mathbf{B N})$. Hence, taking the degree shifts into acount, we have from Proposition 3.17 that

$$
\llbracket K^{T_{+}} \rrbracket_{q}=q \llbracket K^{T_{0}} \rrbracket_{q}-q^{2} \llbracket K^{T_{h}} \rrbracket_{q} .
$$

Likewise, using the other distinguished triangle we obtain

$$
\llbracket K^{T_{-}} \rrbracket_{q}=q^{-2} \llbracket K^{T_{h}} \rrbracket_{q}-q^{-1} \llbracket K^{T_{0}} \rrbracket_{q} .
$$

These two equalities imply the Jones skein relation.

The commutativity of (7.7) can be also checked directly, as there are explicit formulas for all the chain maps, see [BN05]. Although it requires more work, with this approach one can prove Theorem E for the homology with integral coefficients, once a strictly functorial version of the construction due to Chen and Khovanov is used. This approach is used in a following paper. Alternatively, one can fix the signs explicitly as it was done successfully in [GLW15].

One can forget the membrane and work with true annular links, instead of $\mathscr{L i n k s}(\mathbb{A})$. Although the map $K h_{\mathbb{A}_{q}}\left(K^{T}\right)$ is a priori defined only up to an overall power of $q$, making the relation (7.5) problematic, our definition of the map uses a special presentation of the cobordism: as the isotopy sliding $T$ along $\tau$, the opening of $K$. For this particular presentation the power of $q$ is well-defined, so that the Jones relation holds. However, one must be careful when starting to isotope the cobordism.

\subsection{Quantum homology of links in a thickened Möbius band}

Let $\rho \in \operatorname{Diff}(\mathbb{R} \times I)$ be the flip along the interval, i.e. $\rho(x, t)=(-x, t)$. It induces an endobifunctor $\rho_{*}$ on $\mathbf{B N}$. Identifying $(x, 1)$ with $(-x, 0)$ produces a Möbius band $\mathbb{M}$, and, by the argument from the proof of Theorem 2.19, $\operatorname{hTr}^{\oplus}\left(\mathbf{B N}, \rho_{*}\right)$ and $\mathscr{B N}(\mathbb{M})$ are equivalent categories. In an analogy to the case of annulus we define

$$
\mathscr{B} \mathcal{N}_{q}(\mathbb{M}):=\operatorname{hTr}_{q}^{\oplus}\left(\mathbf{B N}(\mathbb{R} \times I), \rho_{*}\right)
$$

The reflection $\rho$ induces also an automorphism of $A^{n}$, which we denote with the same symbol. The following result is immediate from the definition of $\mathbf{F}_{C K}$.

Lemma 7.2. Given an $(m, n)$-tangle $T$ write $T^{\text {flip }}$ for its reflection along the vertical axis. Then

$$
{ }_{\rho} \mathbf{F}_{C K}(T)_{\rho} \cong \mathbf{F}_{C K}\left(T^{f i p}\right)
$$

as $\left(A^{m}, A^{n}\right)$-bimodules.

Thence, we can define a TQFT functor $\mathscr{F}_{\mathbb{M}_{q}}: \mathscr{B}_{\mathcal{N}_{q}}(\mathbb{M}) \longrightarrow \operatorname{g} \operatorname{Mod}(\mathbb{k})$ with the help of the pullback shadow $\left(\mathbf{F}_{C K}\right)^{*} q H H_{0}^{\rho}$. The argument from Proposition 6.6 applied to $q H H^{\rho}$ implies that

$$
q H H_{>0}^{\rho}\left(A^{n}\right)=0 \quad \text { and } \quad q H H_{0}^{\rho}\left(A^{n}\right) \cong \operatorname{coInv}^{\rho}\left(A_{0}^{n}\right),
$$


where the latter is generated by idempotents corresponding to symmetric cup diagrams (i.e. those fixed by $\rho$ ). In particular, if we write $c_{n}$ for the collection of $n$ parallel separating curves, each wrapping $\mathbb{M}$ twice, and $\gamma$ for the nonseparating curve, then

$$
\mathscr{F}_{\mathbb{M}_{q}}\left(c_{n}\right) \cong q H H_{0}^{\rho}\left(A^{2 n}\right) \cong A_{0}^{n} \cong V^{\otimes n} \quad \text { and } \quad \mathscr{F}_{\mathbb{M}_{q}}\left(\gamma \cup c_{n}\right)=0
$$

where $A_{0}^{n} \cong q H H_{0}^{\rho}\left(A^{2 n}\right)$ takes an idempotent $e \in A_{0}^{n}$ to $e \otimes \rho(e) \in A_{0}^{2 n}$. In particular, we are interested only in the subcategory $\mathscr{B} \mathcal{N}_{q}^{e v}(\mathbb{M})$ generated by those collections that do not contain $\gamma$. These collections are characterized by the following property: if $\mu \subset \mathbb{M}$ cuts the band into a square, then each object from $\mathscr{B} \mathcal{N}_{q}^{e v}(\mathbb{M})$ intersects $\mu$ in an even number of points. The argument from Lemma 6.4 adapted to this case shows that $\operatorname{vTr}_{q}^{\oplus}\left(\mathbf{B N}^{e v}, \rho_{*}\right) \longrightarrow \operatorname{hTr}_{q}^{\oplus}\left(\mathbf{B N}^{e b}, \rho_{*}\right)=\mathscr{B} \mathcal{N}_{q}^{e v}(\mathbb{M})$ is an equivalence of categories. The following result is straightforward.

Lemma 7.3. Let $\gamma \subset \mathbb{M}$ be a nonseparating curve. Then there is a commutative diagram of functors

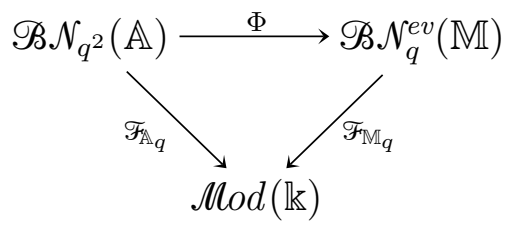

where $\Phi: \mathscr{B}_{q^{2}}(\mathbb{A}) \longrightarrow \mathscr{B} \mathcal{N}_{q}(\mathbb{M})$ is induced by the diffeomorphism $\mathbb{A} \approx \mathbb{M}-\gamma$.

Proof. The functor $\Phi$, seen as $\mathrm{h}_{\operatorname{Tr}_{q^{2}}}^{\oplus}(\mathbf{B N}) \longrightarrow \mathrm{hTr}_{q}^{\oplus}\left(\mathbf{B N}, \rho_{*}\right)$, adds $n$ vertical lines next to an $(n, n)$-tangle and sends a morphism $[T, W]$ to $\left[T \cup T^{f l i p}, W \cup\left(I \times T^{f i p}\right)\right]$. The difference in powers of $q$ appears, because going once through a membrane in $\operatorname{hTr}_{q^{2}}^{\oplus}(\mathbf{B N}) \cong \mathscr{B N}_{q^{2}}(\mathbb{A})$ corresponds to going twice through the membrane in $\operatorname{hTr}_{q}^{\oplus}\left(\mathbf{B N}, \rho_{*}\right) \cong \mathscr{B N}_{q}(\mathbb{M})$.

Each cobordism in $\operatorname{vTr}_{q}^{\oplus}\left(\mathbf{B N}^{e v}, \rho_{*}\right)$ can be decomposed into a composition of cobordisms from the image of $\Phi$ and projective planes with a disk removed, each with a nontrivial curve in $\mathbb{M}$ as its boundary. These can be seen in turn as the saddle cobordisms between a trivial and a nontrivial curve, with the trivial curve capped off. They correspond under the quotient map $(\mathbb{R} \times I) \times I \longrightarrow \mathbb{M} \widetilde{\times} I$ to surfaces $\cup \times I$ and $\cap \times I$, and we denote them by $S_{\cup}$ and $S_{\cap}$ respectively.

Lemma 7.4. $\mathscr{F}_{\mathbb{M}_{q}}\left(S_{\cap}\right): V \longrightarrow \mathbb{k}$ evaluates $v_{+}$and $v_{-}$to $q$ and 1 respectively, whereas $\mathscr{F}_{\mathbb{M}_{q}}\left(S_{\cup}\right): \mathbb{k} \longrightarrow V$ takes 1 to $v_{+}+q^{-1} v_{-}$.

Proof. The module $V:=q H H_{0}^{\rho}\left(A^{2}\right)$ assigned by $\mathscr{F}_{\mathbb{M}_{q}}$ to a nontrivial curve has canonical generators

$$
\left.b_{0}:=\bigcirc \quad \text { and } \quad b_{1}:=\right)(
$$

which corresponds under (7.11) to $v_{+}+q^{-1} v_{-}$and $v_{-}$respectively. The thesis now follows from direct computations. Indeed, the map $\mathscr{F}_{\mathbb{M}_{q}}\left(S_{\cup}\right)$ is given by the sequence

$$
\begin{aligned}
\mathbb{Z}\left[q^{ \pm 1}\right]=q H H_{0}^{\rho}(\mathbb{Z}) & \longrightarrow q H H_{0}^{\rho}\left(\mathbb{Z}, \mathbf{F}_{C K}(\cup) \underset{A^{2}}{\otimes}{ }^{*} \mathbf{F}_{C K}(\cup)\right) \\
& \stackrel{\theta}{\longrightarrow} q H H_{0}^{\rho}\left(A^{2},{ }^{*} \mathbf{F}_{C K}(\cup) \otimes \mathbf{F}_{C K}(\cup)\right) \longrightarrow q H H_{0}^{\rho}\left(A^{2}\right)
\end{aligned}
$$

which takes $1 \in \mathbb{k}$ into $b_{0}$. Dually, $S_{\cap}$ is assigned the sequence

$$
q H H_{0}^{\rho}\left(A^{2}\right) \longrightarrow q H H_{0}^{\rho}\left(A^{2}, \mathbf{F}_{C K}(\cap) \otimes{ }^{*} \mathbf{F}_{C K}(\cap)\right)
$$




$$
\stackrel{\theta}{\longrightarrow} q H H_{0}^{\rho}\left(\mathbb{Z},{ }^{*} \mathbf{F}_{C K}(\cap) \underset{A^{2}}{\otimes} \mathbf{F}_{C K}(\cap)\right) \longrightarrow q H H_{0}^{\rho}(\mathbb{Z})=\mathbb{Z}\left[q^{ \pm 1}\right]
$$

which takes $b_{0}$ and $b_{1}$ to $q+q^{-1}$ and 1 respectively.

The above is enough to compute $\mathscr{F}_{\mathbb{M}_{q}}$ on all morphisms in $\mathscr{B} \mathcal{N}_{q}(\mathbb{M})$. A comparison with the formulas from Section 5.4 shows that $\mathscr{F}_{\mathbb{M}_{q}}$ is a deformation of the APS construction.

Theorem 7.5. Let $\widehat{T}$ be the closure in $\mathbb{M}$ of a $(2 n, 2 n)$-tangle $T$. Then there is an isomorphism

$$
K h_{\mathbb{M}}(\widehat{T}) \cong H H_{\bullet}^{\rho}\left(A^{2 n}, C_{C K}(T)\right)
$$

natural with respect to the chain maps associated to link cobordisms.

\subsection{Annular twistors}

A family of homotopy equivalences $C_{C K}\left(\tau^{\epsilon^{\prime}}\right) \otimes_{A^{n}} C_{C K}(T) \simeq C_{C K}(T) \otimes_{A^{m}} C_{C K}\left(\tau^{-}\right)$was used in Section 7.2 to construct the action of oriented tangles, where $T$ is placed vertically on the membrane. However, one can also place $T$ horizontal and understand the homotopy equivalences as components of a natural endotransformation $\tau_{*}$ of the identity functor on $C_{o m}^{b}(\mathbf{D B})$, where $\mathbf{D B}:=\mathbf{F}_{C K}(\mathbf{B N})$ is the bicategory of diagrammatic bimodules. Therefore, the pair $\left(\mathrm{Id}, \tau_{*}\right)$ is a functor from the endocategory $\left(\mathrm{Com}_{/ h}^{b}(\mathbf{D B}), \mathrm{Id}\right)$ to itself, and we can obtain a new homology theory by pulling back the shadow $\left(\mathbf{F}_{C K}\right)^{*} q H H_{0}$. This motivates the following definition.

Definition 7.6. An annular twistor is a family $\underline{M}:=\left\{M_{\underline{\epsilon}}\right\}$ of chain complexes of $\left(A^{n}, A^{n}\right)$-bimodules, parametrized by finite sequences $\underline{\epsilon}=\left(\epsilon_{1}, \ldots, \epsilon_{n}\right)$ with $\epsilon_{i} \in\{-1,+1\}$, together with natural chain maps

$$
\phi_{T}: M_{\underline{\epsilon}} \underset{A^{m}}{\otimes} C_{C K}(T) \longrightarrow C_{C K}(T) \underset{A^{n}}{\otimes} M_{\underline{\epsilon}^{\prime}}
$$

one per oriented $(m, n)$-tangle $T$ with orientation of input and output encoded by $\underline{\epsilon}$ and $\underline{\epsilon}^{\prime}$ respectively.

Although not stated this way, the chain map (7.15) is a homotopy equivalence. Up to degree shifts, its inverses can be built from $\phi_{T}$ ! together with evaluation and coevalution maps.

Let $L$ be the annular closure of an $(n, n)$-tangle $T$ with orientation of endpoints encoded by a sequence $\underline{\epsilon}$. It follow from Proposition 3.8 that

$$
K h_{\mathbb{A}_{q}}(L, \underline{M}):=q H H_{\bullet}\left(A^{n}, M_{\underline{\epsilon}} \underset{A^{n}}{\otimes} C_{C K}(T)\right)
$$

does not depends on the choice of $T$. We call it the annular homology twisted by $\underline{M}$. When $M_{\underline{\epsilon}}=C_{C K}\left(\tau^{\underline{\epsilon}}\right)$ is the twistor described above, $K h_{\mathbb{A}_{q}}(L, \underline{M})$ is the quantum annular homology of the satellite of $L$ with companion $\widehat{T}$.

\subsection{Generalized annular homology}

There is another generalization of the annular homology, which is very close to twisting. In this section we work in characteristic two, so that the Chen-Khovanov construction is strictly functorial. 
Let us fix a $(1,1)$-tangle $T$ and denote by $T^{!}$its mirror image. Write $V_{T}:=C K h_{\mathbb{A}_{q}}(\widehat{T})$ and $V_{T}^{*}:=C K h_{\mathbb{A}_{q}}\left(\widehat{T}^{!}\right)$for the quantized annular chain complexes of the annular closures of $T$ and $T^{\text {! }}$. They form a dual pair, with evaluation and coevalution maps induced by cobordisms $T \times \cap$ and $T \times \cup$ in $(\mathbb{A} \times I) \times I$ respectively, see Appendix A.4 and Figure 12 for a picture of the evaluation cobordism.

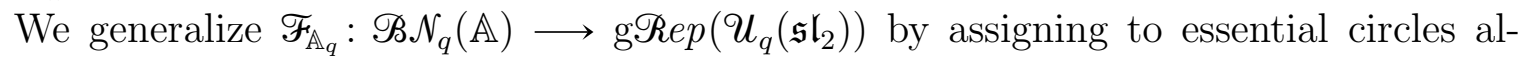
ternatively $V_{T}$ and $V_{T}^{*}$. An annulus with essential boundary is then assigned one of the evaluation of coevaluation maps, and merging a trivial circle to an essential one is determined by the cobordisms merging an unknot to $\widehat{T}$ or $\widehat{T}^{\prime}$. It follows from the functoriality that this produces a well-defined TQFT functor $\mathscr{F}_{\mathbb{A}_{q}}^{T}: \mathscr{B} \mathcal{N}(\mathbb{A}) \longrightarrow \operatorname{Com}_{/ h}^{b}\left(\boldsymbol{U}_{q}\left(\mathfrak{s l}_{2}\right)\right)$, valued in the homotopy category of representations of $\mathcal{U}_{q}\left(\mathfrak{s l}_{2}\right)$.

Definition 7.7. Let $T$ be a $(1,1)$-tangle and choose an annular link $L$ with diagram $D$. The $T$-annular homology $K h_{\mathbb{A}_{q}}^{T}(L)$ of $L$ is the homology of the chain complex $\mathscr{F}_{\mathbb{A}_{q}}^{T} \llbracket D \rrbracket$.

It is tempting to express the above construction using annular twistors. Indeed, one can define $M_{\underline{\epsilon}}$, where $\underline{\epsilon}$ has length $n$, as the alternating tensor product $V_{T} \otimes V_{T}^{*} \otimes V_{T} \otimes \ldots$ of $n$ factors. The duality between $V_{T}$ and $V_{T}^{*}$ can be used to construct a chain map

$$
\nu_{T}: M_{\underline{\epsilon}} \underset{A^{m}}{\otimes} C_{C K}(T) \longrightarrow C_{C K}(T) \underset{A^{n}}{\otimes} M_{\underline{\epsilon}^{\prime}}
$$

for every $(m, n)$-tangle $T$. However, it is not natural. For instance, seeing $T=\bigcirc$ as a composition of a cup and a cap, the composition

$$
M_{0} \otimes C_{C K}(\cup) \underset{A^{2}}{\otimes} C_{C K}(\cap) \stackrel{\nu}{\longrightarrow} C_{C K}(\cup) \underset{A^{2}}{\otimes} M_{2} \underset{A^{2}}{\otimes} C_{C K}(\cap) \stackrel{\nu_{\cap}}{\longrightarrow} C_{C K}(\cup) \underset{A^{2}}{\otimes} C_{C K}(\cap) \otimes M_{0}
$$

is the multiplication by the quantum dimension of $V_{T} \otimes V_{T}^{*}$, the Jones polynomial of $\widehat{T} \# \widehat{T}^{\prime}$. On the other hand, $C_{C K}(\bigcirc)=\mathbb{k}\{-1\} \oplus \mathbb{k}\{+1\}$, suggesting that $\nu_{\bigcirc}=\mathrm{id}$. This seams to be the only issue, because for the decomposition of a zigzag

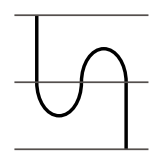

the induced map is a homotopy equivalence due to the relation between the evaluation and coevaluation morphisms.

\section{A Background survey}

The material presented here is widely known, and the main goal of this section is to fix the notation. Bicategories are treated in the excellent paper [Be67], whereas [Lei98] is a brief list of basic definitions. The reader is also referred to [EGNO09], because many results about monoidal categories immediately translates to bicategories.

\section{A.1 Representations of $\mathcal{U}_{q}\left(\mathfrak{s l}_{2}\right)$}

As usual we fix a commutative unital ring $\mathbb{k}$ together with an invertible element $q$. By definition, $\boldsymbol{U}_{q}\left(\mathfrak{s l}_{2}\right)$ is the unital associative $\mathbb{k}$-algebra with generators $E, F, K, K^{-1}$ and relations

$$
K E=q^{2} E K, \quad K K^{-1}=1=K^{-1} K,
$$




$$
K F=q^{-2} F K, \quad K-K^{-1}=\left(q-q^{-1}\right)(E F-F E) .
$$

It is a Hopf algebra with the comultiplication $\Delta: \boldsymbol{U}_{q}\left(\mathfrak{s l}_{2}\right) \longrightarrow \mathcal{U}_{q}\left(\mathfrak{s l}_{2}\right) \otimes \boldsymbol{U}_{q}\left(\mathfrak{s l}_{2}\right)$, the counit $\epsilon: \mathcal{U}_{q}\left(\mathfrak{s l}_{2}\right) \longrightarrow \mathbb{k}$, and the antipode $S: \mathcal{U}_{q}\left(\mathfrak{s l}_{2}\right) \longrightarrow \mathcal{U}_{q}\left(\mathfrak{s l}_{2}\right)$ defined by

$$
\begin{aligned}
\Delta(E) & =E \otimes K+1 \otimes E, & \epsilon(E) & =0, & S(E) & =-E K^{-1}, \\
\Delta(F) & =F \otimes 1+K^{-1} \otimes F, & \epsilon(F) & =0, & S(F) & =-K F \\
\Delta\left(K^{ \pm 1}\right) & =K^{ \pm 1} \otimes K^{ \pm 1}, & \epsilon\left(K^{ \pm 1}\right) & =1, & S\left(K^{ \pm 1}\right) & =K^{\mp 1} .
\end{aligned}
$$

Using this Hopf algebra structure, we can regard the category of finite-dimensional representations of $\boldsymbol{U}_{q}\left(\mathfrak{s l}_{2}\right)$ as a monoidal category with duals. The unit in this monoidal category is given by the trivial representation $V_{0}=\mathbb{k}$, on which $\boldsymbol{U}_{q}\left(\mathfrak{s l}_{2}\right)$ acts by multiplication by $\epsilon(X)$ for any $X \in \mathcal{U}_{q}\left(\mathfrak{s l}_{2}\right)$.

We write $V_{1}:=\operatorname{span}_{\mathbb{k}}\left\{v_{1}, v_{-1}\right\}$ and $V_{1}^{*}:=\operatorname{span}_{\mathbb{k}}\left\{v_{1}^{*}, v_{-1}^{*}\right\}$ for the fundamental representation and its dual. We identify both with the rank two module $V:=\operatorname{span}_{\mathbb{k}}\left\{v_{+}, v_{-}\right\}$ using the isomorphisms

$$
\left\{\begin{array} { c } 
{ v _ { 1 } \longmapsto v _ { + } } \\
{ v _ { - 1 } \longmapsto v _ { - } }
\end{array} \quad \text { and } \quad \left\{\begin{array}{c}
v_{1}^{*} \longmapsto v_{-} \\
v_{-1}^{*} \longmapsto q^{-1} v_{+}
\end{array} .\right.\right.
$$

This equips $V$ with two actions of $\mathcal{U}_{q}\left(\mathfrak{s l}_{2}\right)$ that differ by signs:

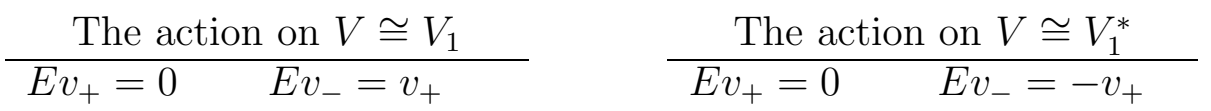

$$
\begin{aligned}
& F v_{+}=v_{-} \quad F v_{-}=0 \quad F v_{+}=-v_{-} \quad F v_{-}=0 \\
& K v_{+}=q v_{+} \quad K v_{-}=q^{-1} v_{-} \quad K v_{+}=q v_{+} \quad K v_{-}=q^{-1} v_{-}
\end{aligned}
$$

The duality between $V_{1}$ and $V_{1}^{*}$ comes with the evaluation and coevaluation maps

$$
\begin{gathered}
e v: V \otimes V \longrightarrow \mathbb{k} \\
v_{+} \otimes v_{+} \longmapsto 0 \quad v_{+} \otimes v_{-} \longmapsto q \\
v_{-} \otimes v_{-} \longmapsto 0
\end{gathered} \quad \frac{\text { coev }: \mathbb{k} \longrightarrow V \otimes V}{1 \longmapsto v_{+} \otimes v_{-}+q^{-1} v_{-} \otimes v_{+}}
$$

that intertwine the action $\boldsymbol{U}_{q}\left(\mathfrak{s l}_{2}\right)$ if $V \otimes V$ is identified with either $V_{1} \otimes V_{1}^{*}$ or $V_{1}^{*} \otimes V_{1}$.

The full subcategory of $\mathcal{U}_{q}\left(\mathfrak{s l}_{2}\right)$ generated by tensor powers of $V$ admits a graphical representation. Let $\mathscr{T} \mathscr{L}$ be the Temperly-Lieb category, the linear category with objects finite collections of points on a real line and morphisms generated by flat loopless tangles, i.e. collection of disjoint intervals in $\mathbb{R} \times I$ with endpoints on the boundary lines. Composition is defined by stacking pictures one onto another and trading each closed component for $q+q^{-1}$. For example,

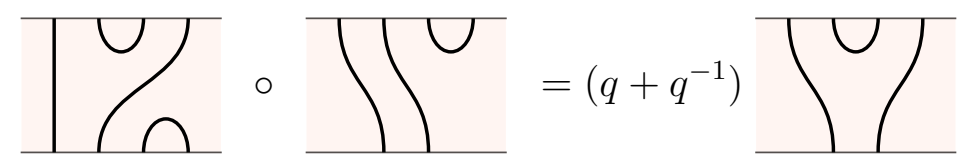

There is a functor $\mathscr{F}_{T L}: \mathscr{T L} \longrightarrow \mathscr{R} e p\left(\mathcal{U}_{q}\left(\mathfrak{s l}_{2}\right)\right)$ that assigns $V^{\otimes n}$ to a collection of $n$ points, whereas caps and cups are sent to the evaluation and evaluation homomorphisms. It is known that $\mathscr{F}_{T L}$ is faithful [Th99]. 


\section{A.2 Knots and tangles}

Let $M$ be an oriented smooth 3 -manifold. A proper 1 -submanifold $T \subset M$ is called a tangle. We call it a link if it has no boundary, and a knot if in addition it has one component. All tangles and links in this paper are assumed to be oriented unless stated otherwise. An isotopy of tangles $T$ and $T^{\prime}$ is a smooth map $\Phi: M \times I \longrightarrow M$ such that each $\Phi_{t}:=\Phi(-, t)$ is a diffeomorphism fixed at the boundary, $\Phi_{0}=\mathrm{id}$, and $\Phi_{1}(T)=T^{\prime}$. If $T$ and $T^{\prime}$ are oriented, then we require that the orientation is preserved by $\Phi_{1}$.

Denote by $-T$ the tangle $T$ with reversed orientation of all its components. A tangle cobordism from a tangle $T_{0}$ to $T_{1}$ is an oriented surface $S \subset M \times I$ with boundary $\partial S=-T_{0} \times\{0\} \cup T_{1} \times\{1\} \cup\left(-\partial T_{0} \times I\right)$. We shall consider cobordisms only up to an isotopy, in which case they form a category: composition is given by gluing cobordisms, and the identity morphism on a tangle $T$ is represented by the cylinder $T \times I \subset M \times I$.

When $M=F \times I$ is a thickened surface, then isotopy classes of oriented tangles in $M$ form a category, with the product induced by stacking, $M \cup M \cong M$, and tangles with tangle cobordisms form a 2-category.

Notation. We shall write $\operatorname{Links}(M)$ for the set of isotopy classes of oriented links in $M$, and $\operatorname{Links}(M)$ for the category of oriented links in $M$ and cobordisms between them. Isotopy classes of oriented tangles in a thickened surface $F \times I$ form a category $\operatorname{Tan}(F)$ with the composition induced by stacking, and similarly cobordisms between tangles in $F \times I$ form a 2-category $\operatorname{Tan}(F)$. We write simply $\mathscr{T}$ an and Tan when $F=\mathbb{R} \times I$.

Assume $M$ is a line bundle over a surface $F$ and consider the projection $M \longrightarrow F$ onto the zero section. It maps a generic tangle $T$ to an immersed collection of intervals and circles $\tilde{T} \subset F$ with only finitely many multiple points, each a transverse intersection of two arcs. A diagram of $T$ is constructed from $\tilde{T}$ by breaking one of the arcs at each double point as follows. When $F$ is oriented, then fibers of $M$ admit a canonical

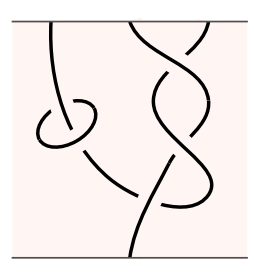
orientation and we break the lower arc at each double point of $\tilde{T}$ (see an example above for $F=\mathbb{R} \times I$ ). In case $F$ is nonorientable, choose a minimal collection of curves $\gamma$ that cuts $F$ into an orientable surface. Then there is a normal field over $F-\gamma$ and we can construct the diagram as before. Then Reidemeister moves and planar isotopies relate diagrams between isotopic tangles if a crossing is switched when moved through $\gamma$ :

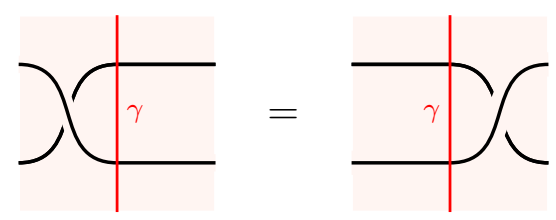

This follows, because the normal field is reversed at points of $\gamma$.

\section{A.3 Constructions on categories}

Below we review definitions of certain constructions on categories that appear throughout the paper.

\section{Additive closure}

We say that a linear category is additive if it has finite direct sums. Each category $\mathscr{C}$ admits the additive closure $\mathscr{C}^{\oplus}$, the smallest additive category containing $\mathscr{C}$. It is constructed by introducing formal direct sums: 
- an object of $\mathscr{C}^{\oplus}$ is a finite sequence $\left(x_{1}, \ldots, x_{r}\right)$ with $x_{i} \in \mathrm{Ob}(\mathscr{C})$, possibly empty,

- a morphism from $\left(x_{1}, \ldots, x_{r}\right)$ to $\left(y_{1}, \ldots, y_{s}\right)$ is a matrix $\left(f_{i j}\right)$ of morphisms $f_{i j} \in$ $\mathscr{C}\left(x_{i}, y_{j}\right)$, and

- composition is defined by the matrix multiplication rule.

There is an inclusion $\mathscr{C} \longrightarrow \mathscr{C}^{\oplus}$, which takes an object $x$ to the 1-element sequence $(x)$. It is an equivalence of categories if $\mathscr{C}$ is already additive.

\section{Idempotent completion}

An endomorphism $p \in \mathscr{C}(x, x)$ satisfying $p \circ p=p$ is an idempotent. We say that $p$ splits if it decomposes $p=s \circ r$ such that $r \circ s$ is an identity morphism. In such a case $r$ is an epimorphism and its codomain is called the image of $p$.

A category is idempotent complete if all its idempotents split. Each category $\mathscr{C}$ admits its idempotent completion $\operatorname{Kar}(\mathscr{C})$, also called also the Karoubi envelope of $\mathscr{C}$, which is the smallest idempotent complete category containing $\mathscr{C}$. It is constructed by taking all idempotents of $\mathscr{C}$ as objects, and defining morphisms from $e$ to $e^{\prime}$ as those morphisms $f$ from $\mathscr{C}$ that $e^{\prime} \circ f \circ e$ is well-defined and equal to $f$. The identity morphism on $e$ is given by the idempotent itself.

\section{Formal complexes and homotopy category}

A formal complexes over a category $\mathscr{C}$ is a sequence of objects and morphisms from $\mathscr{C}$

$$
\left(C^{\bullet}, d\right)=\left(\ldots \longrightarrow C^{i} \stackrel{d^{i}}{\longrightarrow} C^{i+1} \stackrel{d^{i+1}}{\longrightarrow} C^{i+2} \longrightarrow \ldots\right)
$$

satisfying $d^{i+1} d^{i}=0$ at each place. The morphisms $d^{i}$ are called the differential. We say that $\left(C^{\bullet}, d\right)$ is bounded if $C^{i}=0$ except finitely many indices.

A formal chain map from $\left(C^{\bullet}, d\right)$ to $\left(D^{\bullet}, d\right)$ is a collection of morphisms $f^{i}: C^{i} \longrightarrow D^{i}$ fitting into a commuting ladder

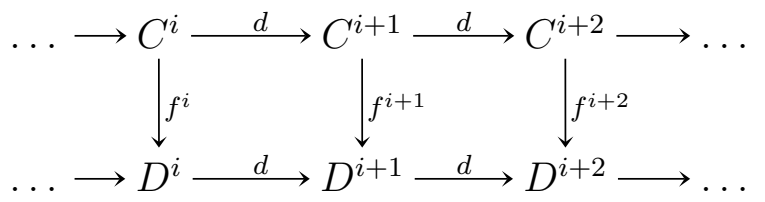

Finally, a formal chain homotopy from $f^{\bullet}$ to $g^{\bullet}$, both chain maps from $\left(C^{\bullet}, d_{C}\right)$ to $\left(D^{\bullet}, d_{D}\right)$, is a collection of morphisms $h^{i}: C^{i} \longrightarrow D^{i-1}$ satisfying $d_{D}^{i-1} \circ h^{i}+h^{i+1} \circ d_{C}^{i}=$ $g^{i}-f^{i}$. In such case we say that $f^{\bullet}$ and $g^{\bullet}$ are homotopic, which we write $f \sim g$.

Formal complexes (reps. bounded formal complexes) and chain maps modulo chain homotopies constitute the homotopy category of complexes $\operatorname{Com}_{/ h}(\mathscr{C})\left(\operatorname{resp} . \mathrm{Com}_{/ h}^{b}(\mathscr{C})\right)$. Isomorphism in these categories are called homotopy equivalences and we usually write $C^{\bullet} \simeq D^{\bullet}$ for complexes that are homotopically equivalent.

The categories $\operatorname{Com}_{/ h}(\mathscr{C})$ and $\operatorname{Com}_{/ h}^{b}(\mathscr{C})$ are triangulated [GM, Wei95], which means that they come with a homological degree shift functor and a collection of distinguish triangles satisfying certain axioms. The degree shift functor is usually denoted by [1] and it shifts a complex leftwards, negating the differential at the same time:

$$
C[1]^{i}:=C^{i+1}, \quad d[1]^{i}:=-d^{i+1} .
$$

Distinguished triangles are of the form

$$
C^{\bullet} \stackrel{f^{\bullet}}{\longrightarrow} D^{\bullet} \stackrel{i n \bullet}{\longrightarrow} \operatorname{cone}^{\bullet}(f) \stackrel{p r^{\bullet}}{\longrightarrow} C[1]^{\bullet},
$$


where cone ${ }^{\bullet}(f)$ stands for the mapping cone of $f$, the formal complex

$$
\operatorname{cone}^{i}\left(f^{\bullet}\right):=C^{i+1} \oplus D^{i}, \quad d^{i}=\left(\begin{array}{cc}
-d_{C}^{i+1} & 0 \\
f^{i} & d_{D}^{i}
\end{array}\right)
$$

The morphisms $i n^{i}: D^{i} \longrightarrow \operatorname{cone}^{i}\left(f^{\bullet}\right)$ and $p r^{i}: \operatorname{cone}^{i}\left(f^{\bullet}\right) \longrightarrow C[1]^{i}=C^{i+1}$ are the inclusion and projection respectively.

\section{A.4 Bicategories}

A bicategory $\mathbf{C}$ is a 'higher level' analogue of a category. It consists of

- a class of objects $\mathrm{Ob}(\mathbf{C})$,

- a category $\mathbf{C}(x, y)$ for each pair of objects $(x, y)$, whose objects and morphisms are called 1- and 2-morphisms respectively and represented by single and double arrows,

- a unit $\operatorname{id}_{x} \in \mathbf{C}(x, x)$ for each object $x$,

- a functor $\circ: \mathbf{C}(y, z) \times \mathbf{C}(x, y) \longrightarrow \mathbf{C}(x, z)$ for each triple of objects $(x, y, z)$, and

- natural isomorphisms

$$
\mathfrak{a}: f \circ(g \circ h) \stackrel{\cong}{\Longrightarrow}(f \circ g) \circ h \quad \mathfrak{l}: \operatorname{id}_{y} \circ f \stackrel{\cong}{\Longrightarrow} f \quad \mathfrak{r}: f \circ \mathrm{id}_{x} \stackrel{\cong}{\Longrightarrow} f
$$

called associators and unitors, which satisfy the pentagon and triangle axioms [Be67].

A bicategory is called strict or a 2-category if the natural isomorphisms are identities. We call $\mathbf{C}$ a locally small bicategory when each category $\mathbf{C}(x, y)$ is small, and $\mathbf{C}$ is small when also $\mathrm{Ob}(\mathbf{C})$ is a set.

Associators and unitors are often omitted for clarity. According to the MacLane's Coherence Theorem [ML98, Chapter VII.2] there is only one way how to insert these isomorphisms back when necessary.

Notation. In this paper we denote categories with calligraphic letters $\mathscr{C}$, $\mathscr{D}$, etc., whereas bold letters $\mathbf{C}, \mathbf{D}$, etc. are reserved for bicategories. Identity morphisms are written as $\mathrm{id}_{x}$, and identity $2-$ morphisms as $\mathbf{1}_{f}$. If $\mathbf{C}$ is a bicategory, then the composition in $\mathbf{C}(x, y)$ is denoted by $*$ and called vertical, whereas $\circ$ is the horizontal composition. These come from the common convention to draw 1 -morphisms horizontally and 2-morphisms vertically, see the diagram to

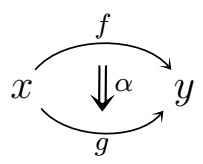
the right of a 2-morphism $\alpha: f \Rightarrow g$.

Choose bicategories $\mathbf{C}$ and $\mathbf{D}$. A bifunctor $\mathbf{F}: \mathbf{C} \longrightarrow \mathbf{D}$ consists of a function of objects $\mathrm{Ob}(\mathbf{C}) \longrightarrow \mathrm{Ob}(\mathbf{D})$ and a collection of functors $\mathbf{C}(x, y) \longrightarrow \mathbf{D}(\mathbf{F} x, \mathbf{F} y)$, together with natural 1-morphisms

$$
\mathfrak{m}: \mathbf{F}(g) \circ \mathbf{F}(f) \Longrightarrow \mathbf{F}(g \circ f) \quad \mathfrak{i}: \operatorname{id}_{\mathbf{F} x} \Longrightarrow \mathbf{F}\left(\operatorname{id}_{x}\right)
$$

satisfying certain coherence axioms. They are called morphisms of bicategories in [Be67], whereas the word homomorphism is reserved for the case when $\mathfrak{m}$ and $\mathfrak{i}$ are invertible. In such case we say that $\mathbf{F}$ is a strong bifunctor.

Choose bifunctors $\mathbf{F}, \mathbf{G}: \mathbf{C} \longrightarrow \mathbf{D}$. A natural transformation $\eta: \mathbf{F} \longrightarrow \mathbf{G}$ is a collection of 1-morphisms $\eta_{x}: \mathbf{F}(x) \longrightarrow \mathbf{G}(x)$, one per object $x \in \mathbf{C}$, and 2-morphisms 
$\eta_{f}: \mathbf{G}(f) \circ \eta_{x} \Longrightarrow \eta_{y} \circ \mathbf{F}(f)$, one per 1-morphisms $f \in \mathbf{C}(x, y)$, such that

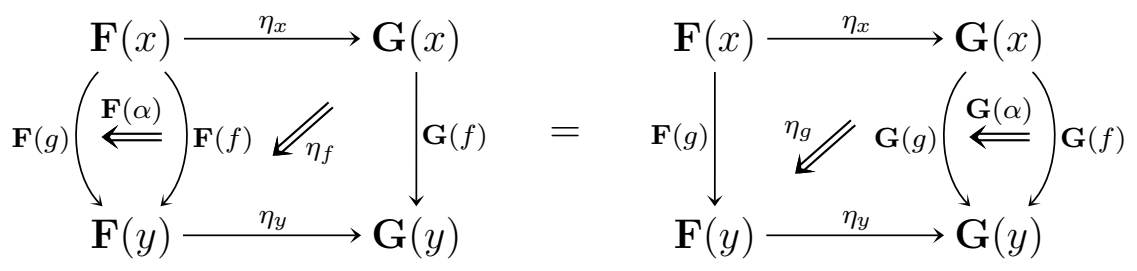

for every 2-morphism $\alpha: f \Longrightarrow g$. Moreover, $\eta_{f}$ must be coherent with all the other canonical 2-isomorphisms (associators, unitors, the structure 2-isomorphisms of $\mathbf{F}$ and $\mathbf{G})$, see [Lei98]. We say that $\eta$ is strong if each $\eta_{f}$ is invertible.

Finally, let $\eta, \nu: \mathbf{F} \longrightarrow \mathbf{G}$ be two natural transformations. A modification $\Gamma: \eta \longrightarrow \nu$ is a collection of 1 -morphisms $\Gamma_{x}: \eta_{x} \longrightarrow \nu_{x}$, such that $\nu_{f} *\left(\mathbf{1}_{\mathbf{G} f} \circ \Gamma_{x}\right)=\left(\Gamma_{y} \circ \mathbf{1}_{\mathbf{F} f}\right) * \eta_{f}$ for every 1 -morphism $f: x \longrightarrow y$.

\section{Duals}

A bicategory $\mathbf{C}$ has left duals if each $f \in \mathbf{C}(x, y)$ admits ${ }^{*} f \in \mathbf{C}(y, x)$ together with coevaluation and evaluation 2-morphisms

$$
\mathrm{id}_{y} \stackrel{\text { coev }}{\Longrightarrow}{ }^{*} f \circ f \quad f \circ{ }^{*} f \stackrel{e v}{\Longrightarrow} \mathrm{id}_{x}
$$

fitting into commuting triangles
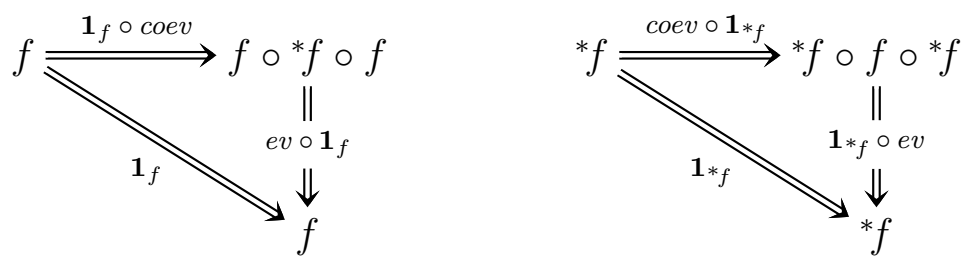

where for clarity associators and unitors are omitted. The morphism ${ }^{*} f$ is called the left $d u a l$ to $f$. We define the right dual $f^{*}$ of $f$ by reversing the order of the horizontal composition in (A.16) and (A.17). If a dual 1-morphism exists, then it is unique up to an isomorphism. In particular, dual pairs are preserved by strong bifunctors.

\section{Examples}

Small categories, functors, and natural transformations form a strong bicategory Cat. A left (resp. right) dual to a functor $F$ is its left (resp. right) adjoint. Hence, not all 1-morphisms in Cat are dualizable.

Tangles in a thickened surface $F \times I$ constitute a bicategory $\operatorname{Tan}(F)$, which objects are finite collections of points in $F, 1$-morphisms are tangles with endpoints on $F \times \partial I$, and $2-$ morphisms are tangle cobordisms. This bicategory has both left and right duals. Indeed, the mirror image $T^{!}$of a tangle $T$, obtained by flipping $F \times I$, is both the left and right dual of $T$. The evaluation 2-morphism is obtained by revolving $T$ in four dimensions along the input surface $F \times\{0\}$, i.e. it is the image of the map $(p, t, s) \longmapsto(p, t \cos (s \pi), t \sin (s \pi))$ with $(p, t) \in T$ and $s \in I$, suitably normalized (see Fig. 12). The coevaluation is defined dually by a rotation along the output surface $F \times\{1\}$.

Rings, bimodules, and bimodule maps form a bicategory Bimod, with horizontal composition given by the tensor product: $N \circ M:=M \otimes_{B} N$ for an $(A, B)$-bimodule $M$ and a $(B, C)$-bimodule $N$. This formula comes from interpreting an $(A, B)$-bimodule $M$ 


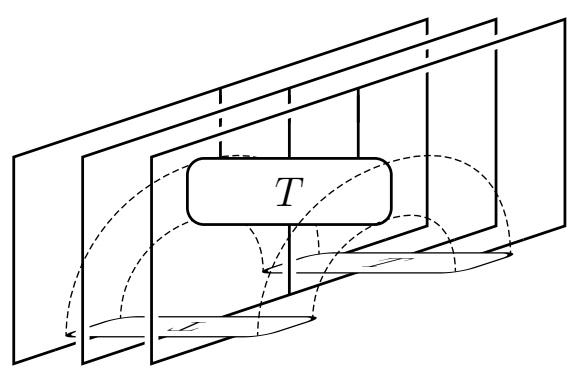

Figure 12: The evaluation cobordism $T T^{!} \Longrightarrow \mathbf{1}_{3}$ for a tangle $T \in \operatorname{Tan}(1,3)$.

as a functor $(-) \otimes_{A} M: \operatorname{Mod}(A) \longrightarrow \operatorname{Mod}(B)$ between the categories of right modules. This bicategory does not have duals. Indeed, an $(A, B)$-bimodule $M$ has a left (resp. right) dual if and only if it is finitely generated and projective as a right $B$-module (resp. left $A$-module). If so, the left and right dual modules are given as the modules of right $B$-linear and left $A$-linear morphisms respectively:

$$
{ }^{*} M:=\operatorname{Hom}_{B}(M, B) \quad M^{*}:=\operatorname{Hom}_{A}(M, A)
$$

For this reason we usually restrict either to Rep (bimodules with left duals) or Birep (bimodules with both left and right duals).

\section{References}

[AF07] M. Asaeda, C. Frohman, A note on the Bar-Natan skein module, Int. J. Math. 18:1225-1243, 2007. Preprint: arXiv:math/0602262.

[APS04] M. M. Asaeda, J. H. Przytycki, and A. S. Sikora, Categorification of the Kauffman bracket skein module of I-bundles over surfaces, Algebr. Geom. Topol. 4:1177-1210, 2004. Preprint: arXiv:math/0409414.

[AGW15] D. Auroux, J. E. Grigsby, and S. M. Wehrli, Sutured Khovanov homology, Hochschild homology, and the Ozsváth-Szabó spectral sequence, Trans. Amer. Math. Soc. 367:7103-7131, 2015. Preprint: arXiv:1303.1986.

[BN05] D. Bar-Natan, Khovanov homology for tangles and cobordisms. Geom. Topol. 9:1443-1499, 2005. Preprint: arXiv:math/0410495.

[BN07] D. Bar-Natan, Fast Khovanov homology. J. Knot Th. Ram. 16:243-256, 2007. Preprint: arXiv:math/0606318.

[BBG18] A. Beliakova, C. Blanchet, A. M. Gainutdinov, Modified trace is a symmetric integral. Preprint: arXiv:1801.00321.

[BGHL14] A. Beliakova, Z. Guliyev, K. Habiro, and A. Lauda, Trace as an alternative decategorification functor, Acta Math. Viet. 39:425-480, 2014. Preprint: arXiv:1409.1198.

[BHLZ14] A. Beliakova, K. Habiro, A. Lauda, and M. Zivkovic, Trace decategorification of categorified quantum $\mathfrak{s l}_{2}$, to appear in Advances in Matematics. Preprint: arXiv:1404.1806. 
[BHPW18] A. Beliakova, M. Hogancamp, K.K. Putyra, S. M. Wehrli, Quantum link homology via trace functor II: Functoriality, in preparation

[BHPW] A. Beliakova, M. Hogancamp, K.K. Putyra, S. M. Wehrli, Colored annular quantum link homology in preparation

[Be67] J. Bénabou, Introduction to bicategories, Reports of the Midwest Category Seminar, Springer Berlin Heidelberg, 1967.

[BZBJ15] D. Ben-Zvi, A. Brochier, D. Jordan, Integrating quantum groups over surfaces: quantum character varieties and topological field theory. Preprint: arXiv:1501.04652.

[Be78] G. M. Bergman, The diamond lemma for ring theory, Adv. Math. 29:178-218, 1978.

[Bla10] C. Blanchet, An oriented model for Khovanov homology, J. of Knot Th. Ram. 19:291-312, 2010. Preprint: arXiv:1405.7246.

[Boe08] J. Boerner, Homology of framed links embedded in thickened surfaces. Preprint: arXiv:0810.5566.

[Bra02] T. Braden, Perverse sheaves on Grassmannians, Canad. J. Math. 54:493-532, 2002. Preprint: arXiv:math/9907152.

[BS11] J. Brundan, and C. Stroppel, Highest weight categories arising from Khovanov's diagram algebra I: cellularity, Mosc. Math. J. 11:685-722, 2011. Preprint: arXiv:0806.1532.

[BS10] J. Brundan, and C. Stroppel, Highest weight categories arising from Khovanov's diagram algebra II: Koszulity, Transform. Groups 15:1-45, 2010. Prepring: arXiv:0806.3472.

[Ca07] C. Caprau, An sl(2) tangle homology and seamed cobordisms, Ph.D. Thesis, University of Iowa, 2007. E-print: arXiv:0707.3051.

[CS98] J. S. Carter, M. Saito, Knotted surfaces and their diagrams, Mathematical Surveys and Monographs 55, American Mathematical Society, Providence, RI, 1998.

[CK14] Y. Chen and M. Khovanov, An invariant of tangle cobordisms via subquotients of arc rings, Fund. Math. 225:23-44, 2014. Preprint: arXiv:math/0610054.

[CMW09] D. Clark, S. Morrison, K. Walker, Fixing the functoriality of Khovanov homology, Geom. Topol. 13:1499-1582, 2009. Preprint: arXiv:math/0701339.

[EGNO09] P. Etingof, S. Gelaki, D. Nikshych, and V. Ostrik, Tensor Categories, Lecture notes, MIT, 2009.

Preprint: http://www-math.mit.edu/ etingof/tenscat.pdf.

[FK97] I. Frenkel and M. Khovanov, Canonical bases in tensor products and graphical calculus for $\mathcal{U}_{q}\left(\mathfrak{s l}_{2}\right)$, Duke Math. Journal 87:409-480, 1997. 
[GM] S. I. Gelfand, Y. I. Manin, Methods of Homological Algebra, Springer Monographs in Mathematics, Springer, 2003.

[GLW15] J. E. Grigsby, T. A. Licata, and S. M. Wehrli, Annular Khovanov homology and knotted Schur-Weyl representations. Preprint: arXiv:1505.04386.

[Ha65] A. Hattori, Rank element of a projective module, NagoyaMath. J. 25:113-120, 1965.

[HPT15] A. Henriques, D. Penneys, and J. Tener, Categorified trace for module tensor categories over braided tensor categories. Preprint: arXiv:1509.02937.

[Jac04] M. Jacobsson, An invariant of link cobordisms from Khovanov homology, Alg. Geom. Top. 4:1211-1251, 2004. Preprint: arXiv:math/0206303.

[Ke98] B. Keller, Invariance and localization for cyclic homology of dg-algebras, J. Pure Applied Alg. 123:223-273, 1998.

[Kh99] M. Khovanov, A categorification of the Jones polynomial. Duke Math. J. 101(3):359-426, 2000. Preprint: arXiv:math/9908171.

[Kh02] M. Khovanov, A functor-valued invariant of tangles. Alg. Geom. Top. 2:665741, 2002. Preprint: arXiv:math/0103190.

[Kh06] M. Khovanov. Link homologies and Frobenius extensions, Fund. Math. 190:179-190, 2006. Preprint: arXiv:math/0411447.

[Le05] E. S. Lee, An endomorphism of the Khovanov invariants, Adv. Math. 197(2):554-586, 2005. Preprint: arXiv:math/0210213.

[Lei98] T. Leinster, Basic bicategories. Prepring: arXiv:math/9810017.

[Lo] J.-L. Loday, Cyclic homology (second ed.), A Series of Comprehensive Studies in Mathematics, Springer, 1998.

[ML98] S. MacLane, Categories for the working mathematician (second ed.), Graduate Texts in Mathematics, Vol. 5, Springer, 1998.

[Mi] D. Miličić, Lectures on Derived Categories. Preprint: http://www.math.utah.edu/ milicic/Eprints/dercat.pdf.

[Mit72] B. Mitchell, Rings with several objects, Adv. Math. 8:1-161, 1972.

[MW10] S. Morrison, K. Walker, The blob complex, Geom. Topol. 16:1481-1607, 2005. Preprint: arXiv:1009.5025.

[PS13] K. Ponto and M. Schulman, Shadows and traces in bicategories, J. Homot. Rel. Struct. 8:151-200, 2013. Preprint: arXiv:0910.1306.

[QR15] H. Queffelec and D. Rose, Sutured annular Khovanov-Rozansky homology. Preprint: arXiv:1506.08188.

[QW18] H. Queffelec and P. Wedrich, Khovanov homology and categorification of skein modules. Preprint. 
[Ras05] J. Rasmussen, Khovanov's invariant for closed surfaces. Preprint: arXiv:math/0502527.

[Rob13] L. Roberts, On knot Floer homology in double branched covers, Geom. Top. 17:413-467, 2013. Preprint: arXiv:0706.0741.

[Rus09] H. M. Russell, The Bar-Natan skein module of the solid torus and the homology of $(n, n)$ Springer varieties, Geom. Dedicata 142:71-89, 2009. Preprint: arXiv:0805.0286.

[Sta65] J. Stallings, Centerless groups - an algebraic formulation of Gottliebs theorem, Topology 4:129-134, 1965.

[Th99] H. Thys, Description topologique des représentations de $\boldsymbol{U}_{q}\left(\mathfrak{s l}_{2}\right)$, Ann. Fac. Sci. Toulouse Math. Vol. 8, No. 4:695-725, 1999.

[Vi02] O. Viro, Remarks on definition of Khovanov homology. Preprint: arXiv:math/0202199.

[Vo15] P. Vogel, Functoriality of Khovanov homology. Preprint: arXiv:1505.04545.

[Wei95] Ch. A. Weibel, An introduction to Homological Algebra, Cambridge University Press, 1995.

[Wei13] Ch. A. Weibel, The K-book. An introduction to K-theory, Grad. Studies in Math. 145, AMS, 2013.

A.B.: University of Zürich, Institute of Mathematics, 8057 Zürich, Switzerland email: anna@math.uzh.ch

K.K.P.: ETH ZÜrich, Institute For Theoretical Studies, 8096 ZÜrich, Switzerland email: krzysztof.putyra@eth-its.ethz.ch

S.M.W.: Syracuse University, Department of Mathematics, Syracuse, NY 13244 email: smwehrli@syr.edu 